UNIVERSIDADE DE SÃO PAULO

INSTITUTO DE FÍSICA DE SÃO CARLOS

\author{
Paulo Hisao Moriya
}

\title{
Collective effects in light scattering from cold strontium clouds
}

São Carlos

2016 

Paulo Hisao Moriya

\section{Collective effects in light scattering from cold strontium clouds}

Thesis presented to the Graduate Program in Physics at the Instituto de Física de São Carlos, Universidade de São Paulo to obtain the degree of Doctor of Science.

Concentration area: Basic Physics

Advisor: Prof. Dr. Philippe Wilhelm Courteille

Original version

São Carlos

2016 
AUTHORIZE THE REPRODUCTION AND DISSEMINATION OF TOTAL OR PARTIAL COPIES OF THIS THESIS, BY CONVENCIONAL OR ELECTRONIC MEDIA FOR STUDY OR RESEARCH PURPOSE, SINCE IT IS REFERENCED.

Cataloguing data reviewed by the Library and Information Service of the IFSC, with information provided by the author

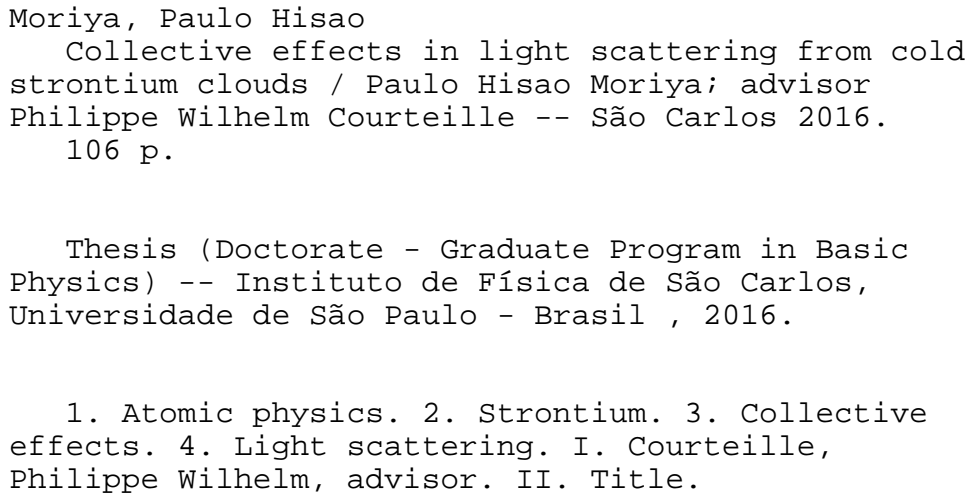

1. Atomic physics. 2. Strontium. 3. Collective effects. 4. Light scattering. I. Courteille, Philippe Wilhelm, advisor. II. Title. 


\section{FOLHA DE APROVAÇÃO}

Paulo Hisao Moriya

Tese apresentada ao Instituto de Física de São Carlos da Universidade de São Paulo para obtenção do título de Doutor em Ciências. Área de Concentração: Física Básica.

Aprovado(a) em: 30/03/2016

Comissão Julgadora

Dr(a). Philippe Wilhelm Courteille

Instituição: (IFSC/USP)

Dr(a). Daniel Varela Magalhães

Instituição: (EESC/USP)

Dr(a). Filippo Ghiglieno

Instituição: (UFSCar/São Carlos)

Dr(a). Celso Jorge Villas Bôas

Instituição: (UFSCar/São Carlos)

Dr(a). Bruno Spolon Marangoni

Instituição: (UFSCar/São Carlos) 

À Carol e Louise. 



\section{Acknowledgements}

I would like to thank Prof. Dr. Philippe Courteille first for the opportunity to work in his Strontium Laboratory, and for his supervision, advice, and trust. His experience is helpful during this work.

To Dr. Michael Holynski, Prof. Dr. Rodrigo Shiozaki and Prof. Dr. Raul Teixeira for their patience and all lessons in experimental techniques, electronics, vacuum technology, optics. To him and his family I wish all the best. I would like to thank as well the Dr. Rodrigo Shiozaki, Dr. Raul Teixeira, Dr. Sharafudeen K. N., the graduate students Fágner, Leonardo, Letícia and Victor, and the visitors Chris Pagett, Faizah Rafique, Djeylan Aktas, Ruben del Aguila and Mohamed Taha Rouabah.

I would like to thank as well all the facilities and technicians maintained by Prof. Dr. Vanderlei Bagnato in the Optics Group. Their knowledge and support in electronics, materials and brazilian bureaucracy were really helpful. In special I would like to thank Liepo crew (Prof. Dr. Daniel Magalhẽs, João Marcelo, Guilherme and Leandro) and the secretaries Isabel, Benê, Cristiane and Adriane. To all the other graduate students for the friendship and exchange of knowledge, my best regards.

I would like to thank the Physics Institute of São Carlos for providing a good place to learn and research. The incredible work made by its Mechanical Workshop group (Ademir, Pereira, João Paulo, Robertinho, Leandro, Gerson, Araldo, Camargo and Ricardo) is crucial to make our crazy ideas reality. Thanks as well to the Prints Workshsp, Optics Workshop, Cryogenics and X-ray Laboratory.

Finally, I would like to thank my wife Ana Carolina and our daughter Louise for making me understand what the word family really means. Without their support and love this journey would be harder. 

"No podemos estar seguros de tener algo por qué vivir si no estamos dispuestos a morir por ella."

Ernesto "Che" Guevara (1928 - 1967) 



\section{Abstract}

MORIYA, P.H. Collective effects in light scattering from cold strontium clouds. 2016. 106 p. Thesis (Doctor in Science) - Instituto de Física de São Carlos, Universidade de São Paulo, São Carlos, 2016.

A cold atomic cloud is a versatile object, because it offers many handles to control and tune its properties. This facilitates studies of its behavior in various circumstances, such as sample temperature, size and density, composition, dimensionality and coherence time. The range of possible experiments is constrained by the specifications of the atomic species used. In this thesis presents the work done in the experiment for laser cooling of strontium atoms, focusing on its stability, which should provide cold and ultracold samples for the study of collective effects in light scattering. From the initial apparatus, innumerous changes were performed. The vacuum system got improved and now reached lower ultra high vacuum due to the pre-baking done to its parts and adding a titanium-sublimation stage. The quadrupole trap were improved by the design and construction of a new pair of coils. The stability of the blue, green and red laser systems and the loss prevention of laser light were improved, giving rise to a robust apparatus. Another important point is the development of homemade devices to reduce the costs and to be used as a monitor of different parts of an cold atoms experiment. From this homemade devices, we could demonstrate a dramatic linewidth narrowing by injection lock of an low cost $461 \mathrm{~nm}$ diode laser and its application to our strontium experiment. In the end, this improved experimental apparatus made possible the study of a new scattering effect, the mirror assisted coherent back-scattering (mCBS).

Keywords: Atomic physics. Strontium. Collective effects. Light scattering. 



\section{Resumo}

Moriya, P.H. Efeitos coletivos em espalhamento de luz de nuvens frias de estrôncio. 2016. 106 p. Tese (Doutorado em Ciências) - Instituto de Física de São Carlos, Universidade de São Paulo, São Carlos, 2016.

Uma nuvem atômica fria é um objeto versátil porque oferece muitas formas de controlar e ajustar suas propriedades. Isso facilita o estudo de seu comportamento em várias circunstâncias, tais como temperatura da amostra, tamanho e densidade, composição, dimensionalidade e tempo de coerência. A extensão de experimentos possíveis é limitada pelas especificações da espécie atômica utilizada. Nessa tese, eu apresento o trabalho feito em um experimento para resfriamento de átomos de estrôncio, focando em sua estabilidade, que deve gerar amostras frias e ultras-frias para o estudo de efeitos coletivos em espalhamento de luz. Do aparato inicial, inúmeras mudanças foram feitas. O sistema de vácuo foi melhorado e agora atinge vácuos ultra altos mais baixos, devido ao pré-cozimento feito em suas partes e a adição de um estágio de sublimação de titânio. A estabilidade e a prevenção de perdas de luz dos sistemas de laser azul, verde e vermelho foram melhorados, levando a um sistema mais robusto. Outro ponto importante é o desenvolvimento de dispositivos caseiros para reduzir os custos e para ser usado como monitor de diferentes partes de um experimento de átomos frios. Destes dispositivos, pudemos demonstrar a redução dramática da largura de linha de um diodo laser de $461 \mathrm{~nm}$ de baixo custo devido a estabilização por injeção. No fim, esse aparato experimental melhorado fez possível o estudo de um novo efeito de espalhamento, o retro espalhamento coerente da luz assistido por espelho.

Palavras-chaves: Física atômica. Estrôncio. Efeitos coletivos. Espalhamento de luz. 



\section{List of Figures}

Figure 1 - Simplified strontium electronic structure with all the relevant cooling transitions and the repumping transitions that are typically used. . . .

Figure 2 - Experimental sequence used in our strontium experiments. The various steps will be explained in the course of the thesis. . . . . . . . . . . . 31

Figure 3 - The current vacuum system which can be divided in. Right: (a) the oven section and (b) the science chamber. Left: The current vacuum system. Top right: the oven section and Bottom left: the science chamber. 33

Figure 4 - Microtubes located in the oven output. Picture taken by using a microscope $($ Zoom $=200 \mathrm{x}) \ldots \ldots \ldots \ldots$. . . . . . . . . . . . . .

Figure 5 - Zeeman slower characteristics. Left: Schematic representation of the Zeeman slower coil and its position dependent number of turns. Right: Measured magnetic field profile of the Zeeman slower coil for a current of 2.01 A . . . . . . . . . . . . . . . . . . .

Figure 6 - Turning off the old MOT coils. Left: by using two MOSFET switches in reverse logic. Right: by directly turning the power supply off. . . . . 36

Figure 7 - Whole CAD project for the coil cooling housing. . . . . . . . . . . . 37

Figure 8 - Measurement of the resistance of the coils winded. Left: coil A. Right: coil B. . . . . . . . . . . . . . . . . . . 38

Figure 9 - Schematic RLC circuit used to measure the coil inductance. Source: By the author. . . . . . . . . . . . . . . . 38

Figure 10 - Measurement of the inductance. Left: Coil A. Right: Coil B. . . . . . 39

Figure 11 - Measurement of the magnetic field at the center of the coils. Left: Coil A. Right: Coil B. . . . . . . . . . . . . . . . . . 39

Figure 12 - Measurement of the magnetic field along the main axis of the coils. Left: Coil A. Right: Coil B. . . . . . . . . . . . . . . . . . . . . . 40

Figure 13 - Turning off response of first coil. Ch1: Hall probe signal; Ch2 and Ch3: controls for MOSFET switch. . . . . . . . . . . . . . . 40

Figure 14 - Temperature evolution of the coils subjected to different currents (chiller flow on). Left: Coil A. Right: Coil B. . . . . . . . . . . . . . . . . . 41

Figure 15 - Measurement of the quadrupole magnetic field along the main axis with $I=3 \mathrm{~A} \ldots \ldots \ldots \ldots \ldots \ldots \ldots \ldots \ldots \ldots \ldots \ldots \ldots \ldots$

Figure 16 - Schematic diagram of the feedback loop. (a) Linear current sensor offset voltage removal part; (b) Operational amplifier comparator stage: computer control signal get compared to control fluctuations and allow current changes;and (c) PID circuit. . . . . . . . . . . . . . . 43

Figure 17 - Schematic representation of the blue laser system, more details in the text. . . . . . . . . . . . . . . . . .

Figure 18 - The blue (center) and green (top right side) laser system. For the blue laser: (1) represents the spectroscopy AOM, (2) the spectroscopy cell, (3) the Zeeman slower beam AOM, (4) the MOT beams AOM, (5) the absorption imaging beam AOM and (6) the green beam AOM. . . . . . 45

Figure 19 - Schematic representation of the green laser system. . . . . . . . . . . 47 
Figure 20 - Absorption spectrum. Left: with the strong saturating beam on (black), where a Lamb dip is exhibited, and off (red); by optimizing the overlap and power ratio of the strong-probe beams allows Lamb dips of order $100 \mathrm{mV}$. Right: Zoom on the Lamb dip obtained in our saturated absorption imaging apparatus. After fitting, the half width at half maximum is equal to 44(1) MHz. . . . . . . . . . . . . . .

Figure 21 - Close up of Fig. 49 showing frequency versus time for the locked and free running laser. One minute corresponds to 6000 measurements. Measured using High-finesse WSU wavelength meter. . . . . . . . . . . . . 50

Figure 22 - Absorption imaging picture and analysis of our cold Sr samples. Here $N_{\text {atoms }}=5 \times 10^{7}$ atoms and a temperature $T \cong 5 \mathrm{mK} . \quad \ldots$. . . . . . 51

Figure 23 - The transmission spectrum of a Fabry Pérot Cavity with a finesse of 60 . The measurement was done by changing the length of the homemade optical cavity described in Table 4 . The cavity length was changed by having one of the mirrors mounted on a piezoelectric transducer and changing the voltage supplied. The data points are an arbitrary measure of the linear change in length of the cavity. The insert is a magnified image of the transmission peak. The asymmetry observed in the shape of the linewidth is believed to be due to higher order modes being transmitted by the optical cavity. . . . . . . . . . . . . . . . . 54

Figure 24 - Homemade scanning etalon. Left: CAD representation of the homemade scanning etalon (a)End part disk; (b) photodiode and its holder; (c) Photodiode disk; (d) Outcoupling piezo/mirror disk; (e) Hollow cylinder shaped piezo; (f) Outcoupling spherical mirror; (g) Main body/spacer; (h) Incoupling mirror; and (i) Incoupling mirror disk. Right: Picture of the final product. . . . . . . . . . . . . . . 55

Figure 25 - One free spectral range a confocal FP coupled to the red laser system. $\quad 56$

Figure 26 - The optical setup for a PDH stabilization. A VCO modulates sidebands onto the laser, which propagates to the cavity, where it is reflected. The reflected beam is separated using a $\lambda / 4$-plate and a polarizing beam splitter (PBS). The intensity is registered by a photodiode (PD) . . . . 57

Figure 27 - Ultra-stable cavity transmitted (black) and reflected (red) signals. . . . 58

Figure 28 - Red laser system. (1) Toptica's DL pro diode laser (master laser), (2) Ultra-stable cavity, (3) Temperature and current stabilized laser diode (slave laser) and (4) $110 \mathrm{MHz} \mathrm{AOM}$ that shifts the light frequency towards resonance. . . . . . . . . . . . . . . . . . . 59

Figure 29 - Schematic representation of the red laser system. . . . . . . . . . . . 60

Figure 30 - Homemade laser diode housing. Left: (a) The aluminum base, (b) collimation tube holder (aluminum), (c) plexiglas protection, (d) Tholabs LTN330 collimation tube, (e) thermistor used as a temperature monitor, (f) Thorlabs TEC 3-6 and (g) D-Sub 9 connectors. Right: Picture. 61

Figure 31 - Heterodyne beat signal between red master and slave laser. . . . . . . . 63

Figure 32 - MOT loading and decay times. Initially all beams are on, then the Zeeman slowing beam is blocked and an exponential decay begins. Fit

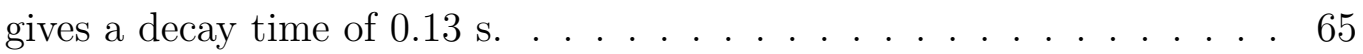


Figure 33 - Transfer of atoms into the magnetic trap for a MOT number of $2 \times 10^{8}$ atoms. The atoms are first cooled in a MOT, and then the repumper blocked. This pumps cold atoms into the magnetically trapable dark state. The light is then switched off for a hold time of at least 100 ms to allow untrapped atoms to vacate the MOT region. A recapture measurement is then performed by pulsing on the repumper and counting the number of atoms that radiate in the MOT. The lifetime of the magnetic trap were found by varying the hold time. (a) Recapture efficiency; and (b) Lifetime measurements. . . . . . . . . . . . . . . . .

Figure 34 - (color online) Optical setup for injection locking. A single mode tunable master laser injects, through an optical isolator, the slave laser diode. The emission spectra of both lasers are simultaneously monitored with a wavemeter, an optical spectrum analyzer (OSA), and a scanning etalon. Also shown is the setup for beat frequency measurement and for saturation spectroscopy on the strong cooling transition of strontium at $461 \mathrm{~nm}$. (ND3: 3dB neutral density filter, PMF: polarization maintaining fiber: AOM: acousto-optic modulator) Also shown is a typical spatial mode profile of the slave laser observed with $200 \mathrm{~mA}$ laser current. . . . . . . . . . . . . . . . . . .

Figure 35 - (color online) (a) Measured total emission power of the free-running laser diode as a function of laser current. The lasing threshold is at a current of $140 \mathrm{~mA}$, the slope efficiency is $1.2 \mathrm{~W} / \mathrm{A}$. (b) Emission spectrum of the free-running laser diode measured with a scanning Fabry-Pérot interferometer at low (black) and high (red and blue) laser currents. . . . . . . . . . . . . . . . .

Figure 36 - (color online) (a) Emission spectrum of the free-running laser diode measured with an optical spectrum analyzer at various laser currents. The temperature of the laser diode was optimized for each current in order to maximize the power in the master frequency. (b) Same as (a) but with $0.5 \mathrm{~mW}$ injected light power from the master laser. (c) Emission spectrum of the slave laser injected with $0.5 \mathrm{~mW}$ measured with a scanning Fabry-Pérot interferometer at low (black) and high (red) laser current. (d) Same as (a) but for a fixed laser current of 477 $\mathrm{mA}$ and varying the injection power. . . . . . . . . . . .

Figure 37 - (color online) Beat frequency spectrum between the master laser frequencyshifted by an AOM at $130 \mathrm{MHz}$ and the injected slave laser. The line is stable and its resolution only limited by the resolution bandwidth (here $100 \mathrm{~Hz}$ ) of the spectrum analyzer and the frequency-noise of the AOM. The noise level is reduced with higher injection power at the exception of two small $1 \mathrm{MHz}$ broad sidebands at $\pm 2 \mathrm{MHz}$ to the left and right of the beat frequency. . . . . . . . . . . . . . . . . . . 71 
Figure 38 - (color online) (a) Saturated absorption spectra recorded with the master laser (lower black curve) and with the injection-locked slave laser (upper red curve). The injection power was $8.6 \mathrm{~mW}$ and the slave laser current $480 \mathrm{~mA}$. Both laser beams had $8 \mathrm{~mW}$ power $(20 \%$ of the saturation intensity) at the entrance of the cell. (b) Single-pass absorption spectrum of the master laser (orange) and the slave laser (other lines) for various injection powers, $1-I_{\text {trans }} / I_{\text {in }}$ versus the master laser detuning $\Delta \ldots \ldots \ldots \ldots \ldots \ldots \ldots$

Figure 39 - (color online) (a) MOT fluorescence recorded while switching the light operating the Zeeman slower: At the time $1.1 \mathrm{~s}$ the master (black) or the slave (red) laser is suddenly irradiated into the Zeeman slower, thus loading the MOT with Sr atoms. In both cases, the master and the slave laser had the same power of $45 \mathrm{~mW}$. (b) Fluorescence measured after the MOT has been fully loaded for various lasers powers irradiated into the Zeeman slower using the master (black) or the slave (red) laser. . .

Figure 40 - Four processes exist in the single scattering regime, hereafter called (iiv), depending on whether the incident light hits first the mirror or the atom, and whether it hits the mirror or not after being scattered. . . .

Figure 41 - Experimental setup: A laser beam passes through a polarizer (Pol) and a wedged beamsplitter (BS) before passing through the atomic cloud a first time. It is then reflected on a virtual mirror (VM) created through two further lenses and a physical mirror at a small angle of $\theta_{0} \sim 1^{\circ}$ with the normal of the mirror. After being reflected, it crosses the cloud again, before being sent by the beamsplitter, together with the light scattered from the atoms, to a CCD camera that images the angular distribution of the light. The top right picture stands for the theoretical fringe pattern for the mCBS set-up, see Suppl. Mat. . . . .

Figure 42 - (a) Averaged image collected on the CCD, after substraction of the background, showing the interference pattern due to the mCBS effect. Inset: fringes' pattern obtained from azimuthal averaging. (b) Mirror position expected from mCBS theory (i.e., $h_{t h r}=p i / k \theta_{0} \Theta_{f}$ ) as a function of the experimental mirror position. This measurement allowed to detect an initial experimental mispositioning of $x_{0}=2.2(1) \mathrm{mm}$, which corresponds to the shifted minimum of the fit $h_{t h r}=A h+x_{0}$ (dashed line); we obtained $A=0.988(12) \ldots \ldots \ldots$. . . . . . . .

Figure 43 - Experimental mCBS contrast (blue triangles), compared to the CBS one from a Strontium cloud (1) (green circles) and from a Rubidium cloud (2) (red stars). The gray areas for the theoretical predictions (for the elastic and inelastic theories (see main text and Suppl. Mat.)) account for the precision within which the experimental parameters are known: $h=8 \pm 0.05 \mathrm{~mm}$ and $\sigma_{z}=0.85 \pm 0.1 \mathrm{~mm}$. The data are a function of the saturation parameter $s$ at the center of the probe beam. The Rb contrast was renormalized so it is 1 in the linear regime, despite the multilevel structure of the atoms. . . . . . . . . . . . . . . . 
Figure 44 - (a) Angular intensity profile in presence (middle black curve) and in absence (top red curve) of the atomic cloud. The deduced atomic fluorescence after the stray light weighted subtraction (see text) $I_{f}(\theta)$ (lower green curve, right vertical axis) presents a locally isotropic background, plus an interference pattern at the center. (b) Transmission coefficient $T$ and (c) background intensity $I_{b g}$ deduced from the fit (see Eq.(6.3) and main text), as a function of the on-axis saturation parameter $s$. The dashed curves are calculated with a simple model for the interaction between our saturated Gaussian laser beam and our Gaussian atomic cloud. . . . . . . . . . . . . . .

Figure 45 - (a) Intensity pattern in the elastic regime (6.13). (b) Azimuthal profile of Fig.(a), showing the fringes profile. The figures can be compared to the measurement presented in Fig. 42(b) . . . . . . . . . . . . . . . .

Figure 46 - Experimental setup for lighthouse effect experiment. The quadrant detector is used because it can measure any vertical and horizontal small displacements. . . . . . . . . . . . . . . . . . 88

Figure 47 - New dodecagon-shaped chamber for the next generation of the experiment. . . . . . . . . . . . . . . . . . .

Figure 48 - Showing the user interface with reasonable settings, and displaying a usable derivative signal. The Scan tab allows control over the SCC110 module. The top middle section is used to set up the PID controllers and Autolock heirarchies. On the top right is the Lock-In control when the settings used for generating a derivative signal can be controlled. The lower portion is a dedicated oscilloscope. The Scope tab shows the direct values of the chosen inputs (Main In and LI out in the image). The Autolock tab shows the inputs over the Autolock range, and allows direct manipulation of the SCC110 (i.e. zooming and panning changes the scan range and scan offset respectively). The dotted line on the oscilloscope is the Autolock cursor - to lock you should position this at the locking point, then right click and select lock. Two useful points: 1) if the oscilloscope stops refreshing make sure Sampling is ticked, and: 2 ) in the lower left there are three rows which have the channel names in them. They are followed by three boxes - clicking on these boxes turns on and off auto-scaling. Source: By the author. . . . . . . . . . . 104

Figure 49 - Showing frequency versus time for the locked laser, free running laser and free running with the Digilock module switched off. One minute corresponds to 6000 measurements. Measured using High-finesse WSU wavelength meter. Source: By the author. . . . . . . . . . . . 106 



\section{List of Tables}

Table 1 - Natural abundance, mass, nuclear spin and scattering length of the four stable strontium isotopes. . . . . . . . . . . . . . . . . . 29

Table 2 - Isotope shifts of the Sr isotopes, given in MHz. All values are referenced to the most abundant isotope ${ }^{88} \mathrm{Sr}$. The hyperfine structure of the fermionic isotope ${ }^{87} \mathrm{Sr}$ is much larger than the isotope shift, which is stated here for an assumed $J=0$ state. . . . . . . . . . . . . 30

Table 3 - Summary of coils characteristics. . . . . . . . . . . . . . . . 42

Table 4 - Measured specifications of our Fabry-Pérot cavities. . . . . . . . . . . 54

Table 5 - Summary of settings for the Digilock interface. . . . . . . . . . . 105 



\section{Contents}

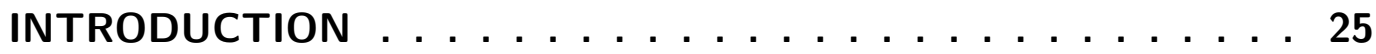

1.1

Cold atoms

Cooperative effects in light scattering

COOLING AND TRAPPING STRONTIUM ATOMS . . . . . . 27

BLUE LASER SYSTEM . . . . . . . . . . . . . . . 33

New coils' project . . . . . . . . . . . . . . . . . . . . 36 lock . 
INJECTION LOCKING OF A LOW COST HIGH POWER LASER

DIODE AT 461 NM . . . . . . . . . . . . . . . . . . 67

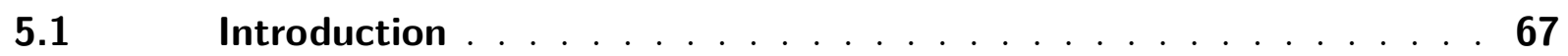

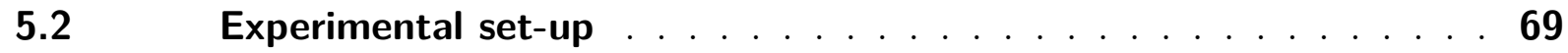

$5.3 \quad$ Characterization of injection locking . . . . . . . . . . . . . 70

$5.4 \quad$ Measurements . . . . . . . . . . . . . . . . 71

$5.5 \quad$ Conclusion . . . . . . . . . . . . . . . . . . . 74

6 COHERENT BACKSCATTERING OF INELASTIC PHOTONS FROM

SINGLE ATOMS AND THEIR MIRROR IMAGES (MCBS) . . . . 75

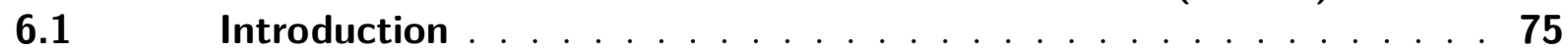

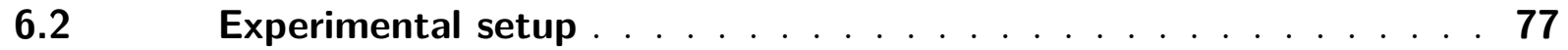

$6.3 \quad$ Linear regime . . . . . . . . . . . . . . . . . . . . . . . . . . . 78

$6.4 \quad$ Saturated regime . . . . . . . . . . . . . . . 78

6.5 Conclusions . . . . . . . . . . . . . . . . . . . 80

6.6 Supplementary material . . . . . . . . . . . . . . 81

6.6.1 Preparation of the Strontium cold cloud . . . . . . . . . . . . . . . 81

$6.6 .2 \quad$ Data analysis: subtraction of the stray light . . . . . . . . . . . . . 82

$6.6 .3 \quad$ Single-scattering theory . . . . . . . . . . . . 83

7 OUTLOOK ON THE NEXT EXPERIMENTS AND THE FUTURE

OF THE EXPERIMENT . . . . . . . . . . . . . . 87

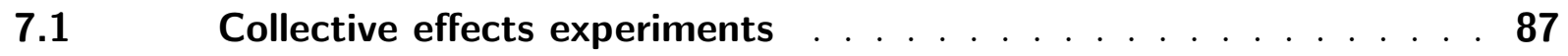

$7.2 \quad$ Experiment next generation . . . . . . . . . . . . . . 88

7.3 Optical dipole trap, Bose-Einstein condesates and Optical lattices . 89

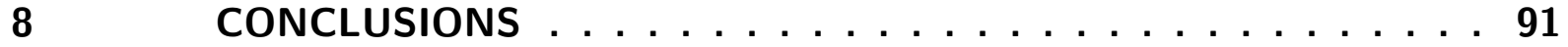

REFERENCES . . . . . . . . . . . . . . . . . 93

$\begin{array}{ll}\text { APPENDIX } & 101\end{array}$

APPENDIX A - TOPTICA DIGILOCK MODULE . . . . . . . . 103

A.1 Overview of software and functionality . . . . . . . . . . 103 


\section{Introduction}

\subsection{Cold atoms}

A cold atomic cloud is a versatile object, because it offers many handles to control and tune its properties. This facilitates studies of its behavior in various circumstances, such as sample temperature, size and density, composition, dimensionality and coherence time. The range of possible experiments is constrained by the specifications of the atomic species used. The most commom experiments are performed using alkali metals such as $\mathrm{Na}(3), \mathrm{K}(4)$, and $\mathrm{Rb}(5)$, which possess a strong cooling transitions with a typical linewidth on the order of $10 \mathrm{MHz}$. More recently, alkaline earth species started to be used, such as $\mathrm{Mg}(6), \mathrm{Ca}(7)$ and $\mathrm{Sr}(8)$, because it offers a set of advantages over the alkali metals. The bosonic isotopes of alkaline earth species have zero hyperfine structure in the ground state, allowing for simpler studies of cold atomic collisions. Additionally, the valence band of these alkaline earth metals, with two electrons, have both spin singlet and triplet energy level. The singlet transition is excellent to be used as a first stage of cooling transition to a thermal atomic beam. The narrow transitions offers interesting opportunities for spectroscopic, precision measurements and the intercombination line transition serves as an excellent cooling transition to $\mu \mathrm{K}$ temperatures without requiring sub-Doppler cooling methods.

\subsection{Cooperative effects in light scattering}

Since Dicke(9) described the phenomenon of superradiance, the cooperative behaviour of particles in scattering of light have attracted huge interest. The development of laser cooling techniques, which created the cold atomic physics, have increased the interest the interest in this kind of phenomena because low temperatures provide almost motionless particles, which decrease decoherence effects and facilitate cooperation between particles.

Among the collective effects, superradiance by an ensemble of atoms is outstanding. It can occours even for one photon where, for example, a cloud of atoms prepared in the timed Dicke state *, has a probability of absorbing the only photon present in the system and create an collective effect that makes the atoms emit a superradiant light pulse in a narrow forward cone(10). This kind of effect give rise to numerous effects such as collective Lamb shift(11) and collective frequency shifts(12).

Interference effects in the propagation of a wave in a disordered medium is also interesting and coherent backscattering is one of the most prominent results obtained when this effect is taken in account. It results in an enhancement of the scattered wave in the retro-direction. This effect was predicted in (13) and observed in the 1980's with $\operatorname{light}(14,15)$.

\footnotetext{
*States initially prepared by absorption of a single photon.
} 


\subsection{Thesis' overview}

The aim of this thesis is the investigation of collective effects in the light scattering. This is done by using cold atomic gases of strontium as a tool due to its unique level structure and the co-existence of broad and narrow cooling transitions will allow us to create samples with different density, volume, shape and temperature in ordered and disordered structures. In order to achieve all the goals, a stable generation of our experimental apparatus is under construction/development and will help us to establish the necessary foundations for the realization of experiments which will study collective effects in the light-matter interaction, specially in what concerns scattering of light.

Chapter 2 reviews some basics on cold atoms, strontium electronic structure and its cooling cycle. Three different wavelengths are needed to cool strontium, one at $461 \mathrm{~nm}$ pumped by a "blue" laser, one at $497 \mathrm{~nm}$ pumped by a "green" laser, and one at $689 \mathrm{~nm}$ pumped by a "red" laser.

The first two lasers, as well as the vacuum system, are discussed in Chapter 3, while the red laser system, which is more complex, is presented in a separate dedicated Chapter 4. In these chapters, I will point out all the work done in order to improve the apparatus and the experiment in general. The two most important results of my Ph.D., are, on one hand an innovation and on the other hand the observation of novel phenomenon in cooperative scattering in a cold atomic sample.

The innovation, i.e. the demonstration of dramatic linewidth narrowing by the injection lock of an low cost $461 \mathrm{~nm}$ diode laser and its application to our strontium experiment, in shown in chapter 5.

The new scattering effect, which is the observation and characterization of mCBS (mirror assisted coherent backscattering) is discussed in chapter 6. Finally, an outlook on the future of the experiment, in chapter 7 and conclusions, in chapter 8, are presented. 


\section{Cooling and trapping strontium atoms}

\subsection{Basic concepts}

In this section, I intend to give a brief conceptual introduction, after which I discuss how these techniques applied to strontium, which needs first a traditional Doppler cooling to pre-cool and collect an atomic sample at $4 \mathrm{mK}$ from a thermal beam source and second transfer them to a narrow line Doppler cooling using the ${ }^{1} S_{0}-{ }^{3} P_{1}$ transition to reach photon-recoil-limited temperatures below $1 \mu \mathrm{K}$. More details can be found in numerous reviews and research articles (16-22).

Momentum conservation is the main characteristic that should be considered. Absorption and emission of a photon by an atom leads to a change of $\mathbf{p}_{p h}=\hbar \mathbf{k i n}$ the atomic momentum, where $\hbar$ is Planck's constant divided by $2 \pi$ and $\mathbf{k}$ is the light wave vector (here $|\mathbf{k}|=2 \pi / \lambda$ and $\lambda$ is the wavelength of the light).

If one consider a two level atom, with mass $m$, with velocity $\mathbf{v}$ propagating in the opposite direction of the light, the atomic momentum is changed from $\mathbf{p}_{\text {atom }}=m \mathbf{v}$ to $\mathbf{p}_{\text {atom }}=m \mathbf{v}+\hbar \mathbf{k}$. After some time, the atom will experience an spontaneous decay process (or the emission of a photon), resulting in another momentum change. Due to spontaneous emission isotropic behavior the atom need a repeated photon absorption-emission process to reduce the atomic velocity $\mathbf{v}$ and cool itself.

If one considers the atomic levels separated by $\hbar \omega_{0}$, where $\omega_{0}$ is the angular frequency of the transition, the frequency of the absorbed light is:

$$
\omega_{\text {absorp }}=\omega_{0}+\mathbf{k} \cdot \mathbf{v}+\frac{\hbar \mathbf{k}^{2}}{2 m}
$$

where the Doppler shift (second term), due to the moviment of the atoms, and the the recoil frequency (third term) are taken in account. In the same way, the frequency of a emitted photon by spontaneous emission is given by:

$$
\omega_{e m i t}=\omega_{0}+\mathbf{k}^{\prime} \cdot \mathbf{v}^{\prime}-\frac{\hbar \mathbf{k}^{2}}{2 m}
$$

So, the average change in kinetic energy of the atom, for multiple events, is:

$$
\Delta E=-\hbar\left(\omega_{\text {abs }}-\omega_{\text {emit }}\right)=\mathbf{k} \cdot \mathbf{v}+2 \frac{\hbar \mathbf{k}^{2}}{2 m} .
$$

The Eq. 2.3 has two solutions for reduction in the kinetic energy: $\mathbf{k} \cdot \mathbf{v}<0$ (antiparallel) and $\mathbf{k} \cdot \mathbf{v}>2 \frac{\hbar \mathbf{k}^{2}}{2 m}$.

In the real life, when one wants to laser cool a thermal atomic beam it is important to take in account the linewidth of the transition. For strontium, for example, the main transitions used are $30.5 \mathrm{MHz}$ and $7.5 \mathrm{kHz}$, corresponding to speeds of $\mathrm{m} / \mathrm{s}$ to $\mathrm{mm} / \mathrm{s}$. But the Sr atoms in the thermal beam (around $600{ }^{\circ} \mathrm{C}$ ) have initial velocities of hundreds of $\mathrm{m} / \mathrm{s}$. At such velocities, the photons are absorbed in a non-resonant process (Doppler 
shift). Among all the approaches, the most popular is a position dependent magnetic field which generates a spatially dependent Zeeman shift that compensates the change in Doppler shift as the atoms are slowed.

Once cooled, the atoms can have its velocities even more decreased and be trapped in a combination of counter-propagating laser beams and a gradient of magnetic field, created by a pair of coils in anti-Helmholtz configuration. This tool is named MagnetoOptical Trap (MOT), and what the light and magnetic fields creates is an restorative force which brings the atomic velocity towards zero in the center of the geometry. This process is not perfect because light keep being absorbed and the resulting spontaneous emission creates velocity fluctuations which heat the components of the trap. This competition between processes of cooling and heating limits the final temperature of the atom (18). This limit is named Doppler-cooling temperature and is given by:

$$
k_{B} T_{\min }=\frac{h \gamma}{2}
$$

where $k_{B}$ is Boltzmann's constant. The laser cooling described above can be applied to multiple atoms, which generate samples of millions or billions of cold atoms.

Only magnetic fields can be used as well to trap atoms. By using the gradient of magnetic field generated by a pair of anti-Helmholtz coils the spatially dependent Zeeman shift pushes atoms toward the trap minimum (23).

\subsection{Strontium atoms}

Strontium is an alkaline earth metal with 38 electrons and is Helium-like, i.e. it has two valence electrons that significantly interact with each other. The electronic structure of ${ }^{88} \mathrm{Sr}$ is shown in Fig.1.

The scheme only shows the excitations of one of the two electrons in the fifth shell ${ }^{1} S_{0}$, while the other remains on the $5 s$-level. All other excitations, including two electrons or lower electrons are of higher energy and are not relevant for this experiment. The electronic valence eigenstates can be divided into singlet-states, $S=0$, and triplet states, $S=1$, due to the three different orientations of the spin relative to the total orbital angular momentum. The ${ }^{1} P_{1}$-state appears as we are already populating the fifth shell, where there is no degeneracy of orbital angular momentum. The transition from the ground state is strongly coupled with a linewidth of $30.8 \mathrm{MHz}$ to a light field with a wavelength of $460.73 \mathrm{~nm}$. The corresponding triplet ${ }^{3} P_{J}$ with one excited electron, and thus antisymmetric spacial wave function and with less electron repulsion, forms the metastable ground state of the triplet system.

There are four stable Sr isotopes, of which three are bosonic $\left({ }^{88} \mathrm{Sr},{ }^{87} \mathrm{Sr},{ }^{84} \mathrm{Sr}\right)$ and one fermionic $\left({ }^{87} \mathrm{Sr}\right)$. Their natural abundance, mass, nuclear spin and scattering length, are summed up in Table 1. The frequency shifts between the transitions of the isotopes are listed in Table 2. 


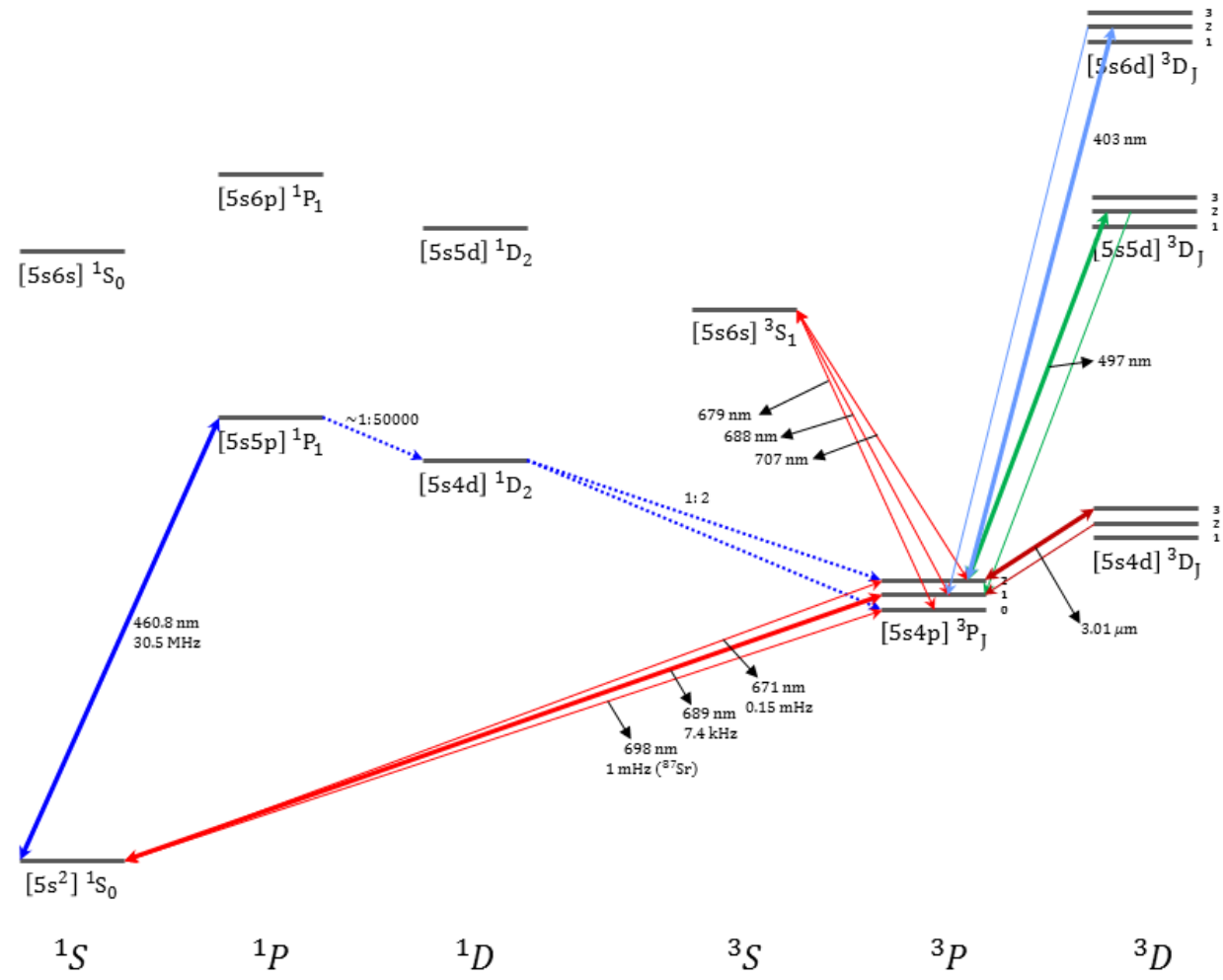

Figure 1 - Simplified strontium electronic structure with all the relevant cooling transitions and the repumping transitions that are typically used.

Source: By the author.

Table 1 - Natural abundance, mass, nuclear spin and scattering length of the four stable strontium isotopes.

\begin{tabular}{ccccc}
\hline Isotope & Abundance & Mass [in u] & Nuclear spin & Magnetic moment \\
\hline \hline${ }^{88} \mathrm{Sr}$ & $82.58 \%$ & 87.905619 & 0 & \\
${ }^{87} \mathrm{Sr}$ & $7.00 \%$ & 86.908884 & $9 / 2$ & -1.093 \\
${ }^{86} \mathrm{Sr}$ & $9.86 \%$ & 85.909267 & 0 & \\
${ }^{84} \mathrm{Sr}$ & $0.56 \%$ & 83.913430 & 0 & \\
\hline
\end{tabular}

Source: By the author.

\subsection{Repumping in strontium}

As pointed in the previous section, strontium broad blue transition is not closed. The atoms decay from the ${ }^{1} P_{1}$ state, with a branching ration of $1: 50000$, to the ${ }^{1} D_{2}$ state. It further decay to the metastable ${ }^{3} P_{2}$ (branching ratio $2: 1$ ), which has a long lifetime, or to the ${ }^{3} P_{1}$, where it decay quickly to the ground state. Atoms in this state can be trapped into the magnetic trap and to be recovered they need to be pumped in higher states to reach the short lived ${ }^{3} P_{1}$ state. A list of possible states that can be used is presented below. 
Table 2 - Isotope shifts of the Sr isotopes, given in MHz. All values are referenced to the most abundant isotope ${ }^{88} \mathrm{Sr}$. The hyperfine structure of the fermionic isotope ${ }^{87} \mathrm{Sr}$ is much larger than the isotope shift, which is stated here for an assumed $J=0$ state.

\begin{tabular}{cccccc}
\hline & ${ }^{1} S_{0} \rightarrow{ }^{1} P_{1}$ & ${ }^{1} S_{0} \rightarrow{ }^{3} P_{1}$ & ${ }^{3} P_{1} \rightarrow{ }^{3} S_{1}$ & ${ }^{3} P_{2} \rightarrow(5 s 5 d)^{3} D_{2}$ & ${ }^{3} P_{2} \rightarrow(5 s 5 d)^{3} D_{3}$ \\
\hline \hline${ }^{88} \mathrm{Sr}$ & 0 & 0 & 0 & 0 & 0 \\
${ }^{87} \mathrm{Sr}$ & -46.5 & 62.15 & 54 & & \\
${ }^{86} \mathrm{Sr}$ & -124.8 & 163.81 & $\approx 100$ & -47.5 & -46.6 \\
${ }^{84} \mathrm{Sr}$ & -270.8 & -351.49 & $\approx 200$ & -91.8 & -91.6 \\
\hline
\end{tabular}

Source: By the author.

1. $5 s 6 s^{3} S_{1}$ : atoms pumped in this state, by using a $679 \mathrm{~nm}$ laser, can also decay into the ${ }^{3} P_{0}$ clock state, which has a long lifetime (to recover this atoms one should use a $707 \mathrm{~nm}$ laser;

2. 5 snd ${ }^{3} D_{2}$ : In these transition, atoms can decay back down into the ${ }^{3} P_{2}$ state to be repumped again or decay into the desired ${ }^{3} P_{1}$ state. For $n=4,5$ and 6 the lasers to be used are $3.0 \mu \mathrm{m}, 497 \mathrm{~nm}$ and $403 \mathrm{~nm}$, respectively;

\subsection{Cooling cycle}

Strontium has a very advantageous electronic structure for laser cooling. It offers a $30.8 \mathrm{MHz}$ broad transition that is almost closed and a closed narrow $7.6 \mathrm{kHz}$ transition, which is thin enough to cool the atoms to the recoil limit, but strong enough to sustain the atoms against gravity. There are different ways how to take advantage of the variety of transitions. A possible sequence (the one employed in this work) for cooling strontium from a hot vapor to quantum degeneracy can be divided in the following steps (2 shows our experimental sequence):

1. Producing strontium vapor in an oven at around $550{ }^{\circ} \mathrm{C}$ and collimate it via microtubes;

2. Slowing the thermal atomic beam from the oven using a spin-flip Zeeman slower at the $30.8 \mathrm{MHz}{ }^{1} S_{0} \rightarrow{ }^{1} P_{1}$ transition;

3. Loading the first stage magneto optical trap (MOT) using the same transition. There is only a small loss channel via the ${ }^{1} D_{2}$ level that eventually decays into the triplet ${ }^{3} P_{2}$. Even though these states are not cooled further at this point they are trapped within the magnetic field gradient due to their magnetic moment. This means that while operating this first stage MOT the metastable ${ }^{3} P_{2}$ accumulates a population of cooled atoms;

4. Repumping: A laser beam at $497 \mathrm{~nm}$ excites atoms in the ${ }^{3} P_{2}$ to the ${ }^{3} D_{2}$ state, from where they decay into the ${ }^{3} P_{1}$ state and back into the ${ }^{3} P_{2}$. Within a short time the population from the ${ }^{3} P_{2}$ state is completely transferred to the ${ }^{3} P_{1}$ state;

5. The second stage MOT using the $7.6 \mathrm{kHz}{ }^{1} S_{0} \rightarrow{ }^{3} P_{1}$ transition; 
6. Transfer to a $1064 \mathrm{~nm}$ crossed beam optical trap and evaporation of hot atoms by trap depth;

7. Observation of Bose-Einstein condensation and eventually transfer to other traps, e.g. an optical lattice.

The various steps of the sequence are detailed in the following sections.

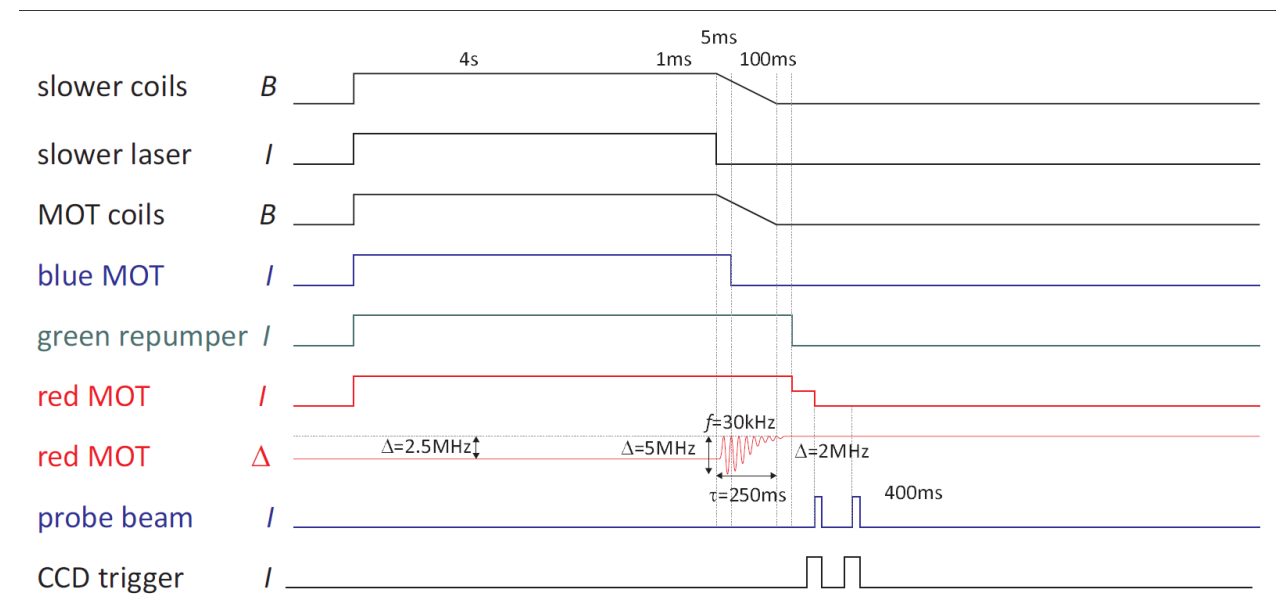

Figure 2 - Experimental sequence used in our strontium experiments. The various steps will be explained in the course of the thesis.

Source: By the author. 



\section{Blue laser system}

A stable experimental setup is required in order to produce cold, ultracold and Bose-Einstein condensates of Strontium. The experimental setup is divided two parts: A low vacuum section, where an oven produces a collimated atomic beam; and a high vacuum section including the Zeeman slower and the science chamber, where the atoms are trapped and experiments can be performed. In the following sections the cooling cycle and current apparatus will be described.

\subsection{The vacuum system}

The layout of the vacuum system follows traditional cold atoms experiments. The system, shown in Figs.3, is divided into two sections, separated by a gate valve: the oven section contains the actual oven and differential pumping stages to provide a high pressure gradient, and the UHV chamber is where the experiments take place. This concept allows to quickly refill or change the strontium oven without breaking the ultra-high vacuum of the UHV chamber.
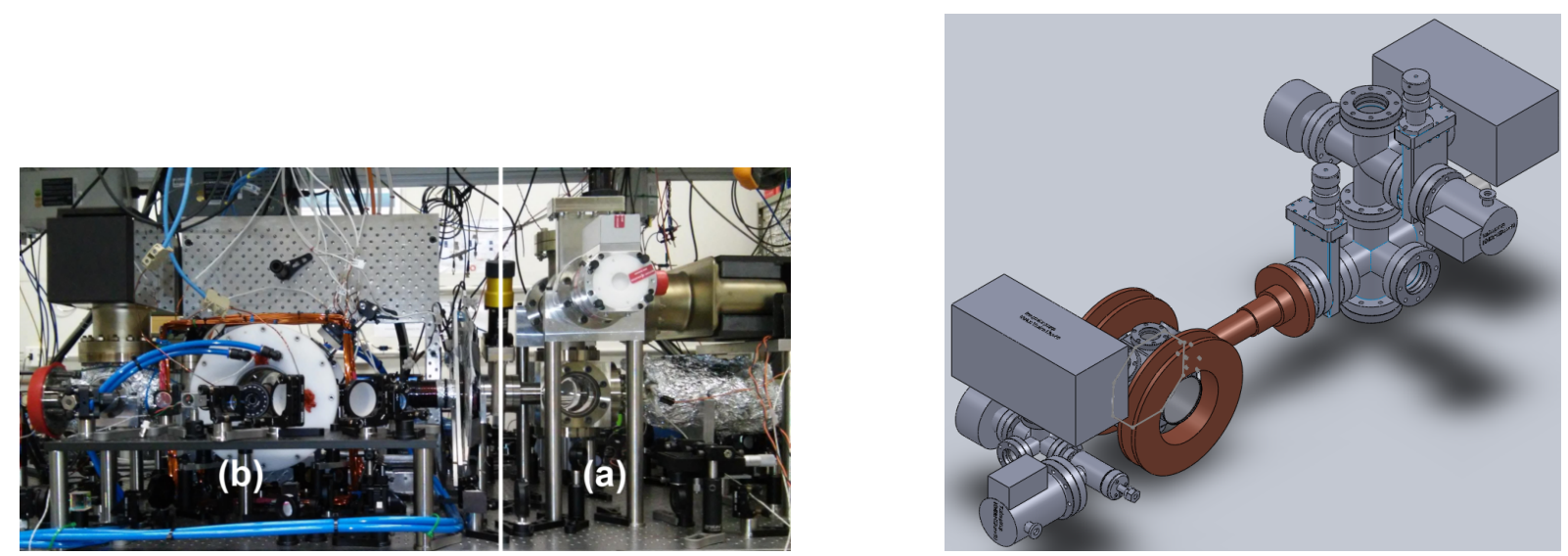

Figure 3 - The current vacuum system which can be divided in. Right: (a) the oven section and (b) the science chamber. Left: The current vacuum system. Top right: the oven section and Bottom left: the science chamber.

Source: By the author.

\subsubsection{Oven section}

The oven is a $132 \mathrm{~mm}$ stainless steel tube with a built-in CF40 flange and it is divided in strontium cell chamber and microtubes. When the first is heated to $550{ }^{\circ} \mathrm{C}$, it produces a strontium vapor, that will produce a collimated atomic beam, after the passage through the microtubes. This beam travels down the main axis of the vacuum chamber. The microtubes, as in Fig. 4, are made from 30G commercial injection needles 
(internal and external diameters are equal to $130 \mu \mathrm{m}$ and $300 \mu \mathrm{m}$, respectively) cut in 8 $\mathrm{mm}$ pieces by a pulsed high power laser and they are all stacked into a circular holder. It is maintained at $600{ }^{\circ} \mathrm{C}$ to prevent clogging by strontium atoms. The oven produces a atomic beam whose flux is $10^{14}$ atoms per second and divergence of $70 \mathrm{mrad}$. In this stage, the average velocity of the atoms is around $400 \mathrm{~m} / \mathrm{s}$.

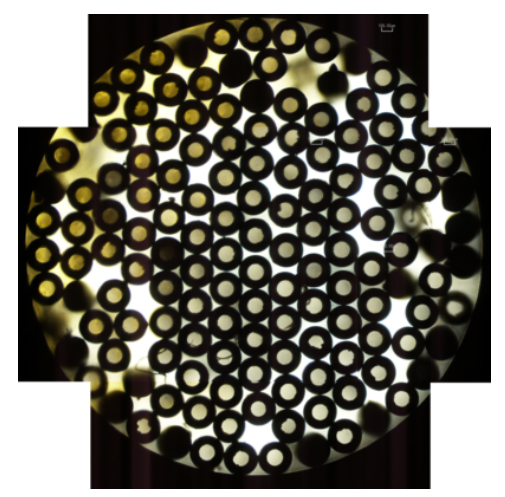

Figure 4 - Microtubes located in the oven output. Picture taken by using a microscope (Zoom $=200 \mathrm{x})$.

Source: By the author.

The oven is attached to a CF63 cube which contains windows for transverse cooling and atomic beam spectroscopy. Through the other connections we attach a $40 \mathrm{l} / \mathrm{s}$ ion pump and a valve for initial pumping. The Sr emanating from the oven and deposited on the walls of the vacuum chamber acts as a supreme getter material and carries a large share in establishing a good vacuum already in the oven section. Counter-intuitively, the vacuum quality in the oven section improves when the oven is turned to higher temperatures. A gate valve allows for a separation between the oven section and the UHV section, which is important for filling or exchanging the oven. This entire oven section is mounted on a number of adjustable posts and connected to the UHV chamber by a bellow, and can thus be moved and rotated in all six degrees of freedom. In this way, the oven flux can be directed precisely into the MOT region.

\subsubsection{Ultra-high vacuum (UHV) section}

The UHV section begins with the Zeeman slower (ZS), which is about $280 \mathrm{~mm}$ long with the inner diameter which changes along the Zeeman slower (it starts with an 12 mm diameter section, followed by 10, 8, 6 and $4 \mathrm{~mm}$ ones) and acts as another differential pumping stage. The back part is connected, via the gate valve, with the low vacuum section, and the front part is connected to the science chamber. One $40 \mathrm{l} / \mathrm{s}$ ion-getter pump and a valve for initial pumping are connected in the opposite side of the UHV chamber where the Zeeman slower tube is connected. The atomic beam impinges on a CF40 sapphire window through which the Zeeman slower beam enters to cool down the atomic beam moving towards the science chamber. Precaution has been taken to avoid deposition of a reflective layer of $\mathrm{Sr}$ on this window. First, sapphire is used instead of standard glass as its surface is less sensitive to etching of aggressive materials. Second, the window is heated to $150{ }^{\circ} \mathrm{C}$ to increase the chances of atoms to detach from the surface again. Absence of heating create a reflective coating with a diameter of about the Zeeman 
Slower laser beam waist which cannot be removed at reasonable temperatures of up to around $300^{\circ} \mathrm{C}$.

\subsection{The magnetic fields}

Magnetic fields are needed to slow down the hot atomic beam, in the Zeeman Slower, and to trap them at the science chamber. The Zeeman slower is a common method for slowing neutral atom beams $(24,25)$ which provides a spatially varying magnetic field that compensates the Doppler shift caused by the moving atoms in order to optimize the deceleration. It provides at each point along the path a magnetic field-induced Zeeman shift to cancel the corresponding Doppler shift. The current Zeeman Slower has a magnetic field profile based in a spin-flip configuration, i.e., the field flips its orientation along the symmetry axis.
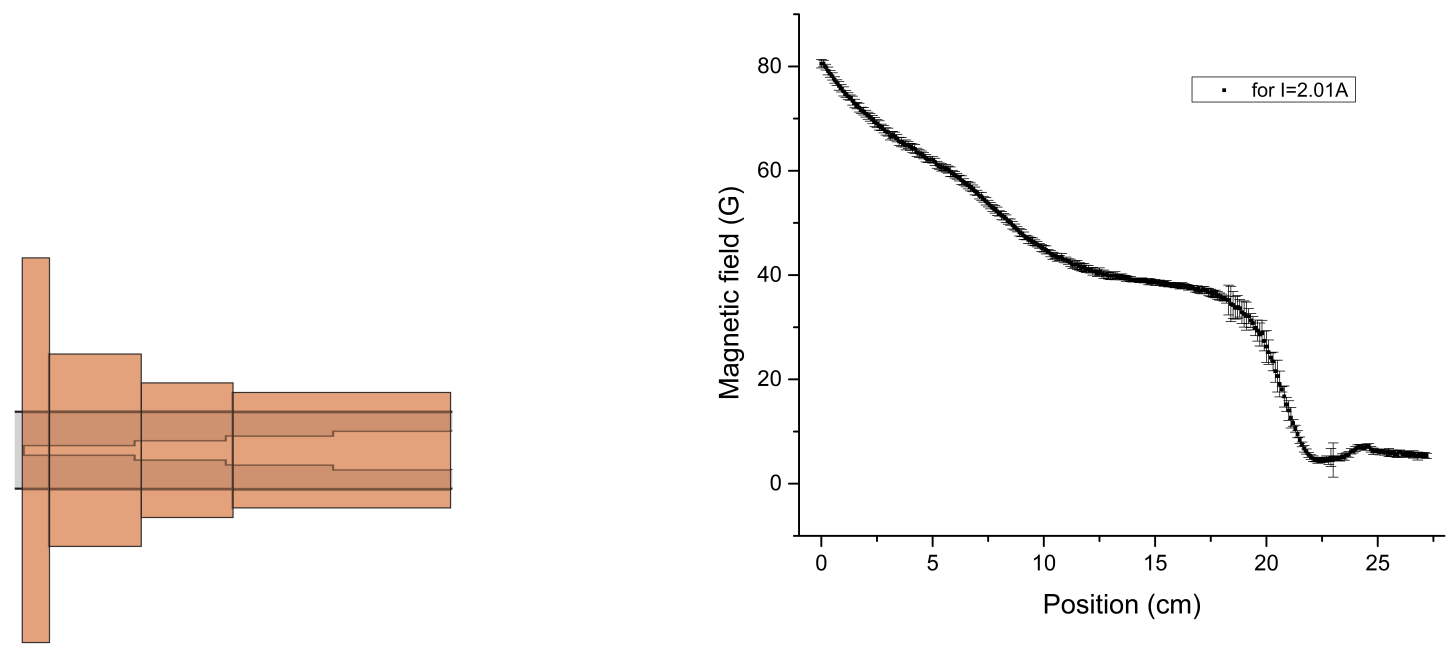

Figure 5 - Zeeman slower characteristics. Left: Schematic representation of the Zeeman slower coil and its position dependent number of turns. Right: Measured magnetic field profile of the Zeeman slower coil for a current of $2.01 \mathrm{~A}$.

Source: By the author.

In the current generation of our experiment, this profile, determined by a simulation of the coils' magnetic field, is achieved by a compact design which starts with a single coil whose number of turns is position dependent, with sucessive layers of $95,35,12,11$, 10 and 8 turns, as in Fig. 5. It is then superimposed with the quadrupole field generated in the science chamber by a pair of anti-Helmholtz coils to achieve the zero-crossing and the spin-flip configuration. For fabricating this coil, a $5 \mathrm{~mm} \times 1 \mathrm{~mm}$ rectangular cross-section shaped copper wire was used to facilitate the stacking of wires. The magnetic field profile of the Zeeman slower (see Fig. 5) was measured for $I=2.01$ A.

The magnetic field gradient necessary to complete the ZS profile at the end of the deceleration process and trap atoms in the science chamber is obtained by a pair of coils in anti-Helmholtz configuration. The magnetic field is stabilized using a feedback loop control and further details can be found in the next section 3.3. 


\subsection{New coils for quadrupole magnetic field}

\subsubsection{Old coils}

The quadrupole magnetic field is generated by a pair of coils in anti-Helmholtz configuration. It is responsible for the magnetic trap and, together with the light fields, it cools and traps atoms in magneto-optical traps (MOT). In our experiments, these coils should be able to generate maximum magnetic field gradients of $70 \mathrm{G} / \mathrm{cm}$, around the center of the trap, when 30 A flows in each of them. Such high current generates $600 \mathrm{~W}$ in each coil, which requires an active dissipation to water in order to avoid overheating. Futhermore, we should be able to switch off quickly magnetic field when the atoms are transfered to the narrow red transition.

In the old version, one coil was composed by 5 independent layers of $40-45$ turns connected in series. The coils rested on a aluminum housing and most of the cooling were by air. This first versions of the quadrupole magnetic field presented some drawbacks:

1. Poor active cooling for the power generated by the coils;

2. Coils housing made of aluminum, which generates Eddy currents;

3. Short circuits between the layers of the coils reduced the number of turns - mainly caused by overheating in the coils;

4. High switch off time of tens of miliseconds - mainly caused by Eddy currents (see Fig. 6).
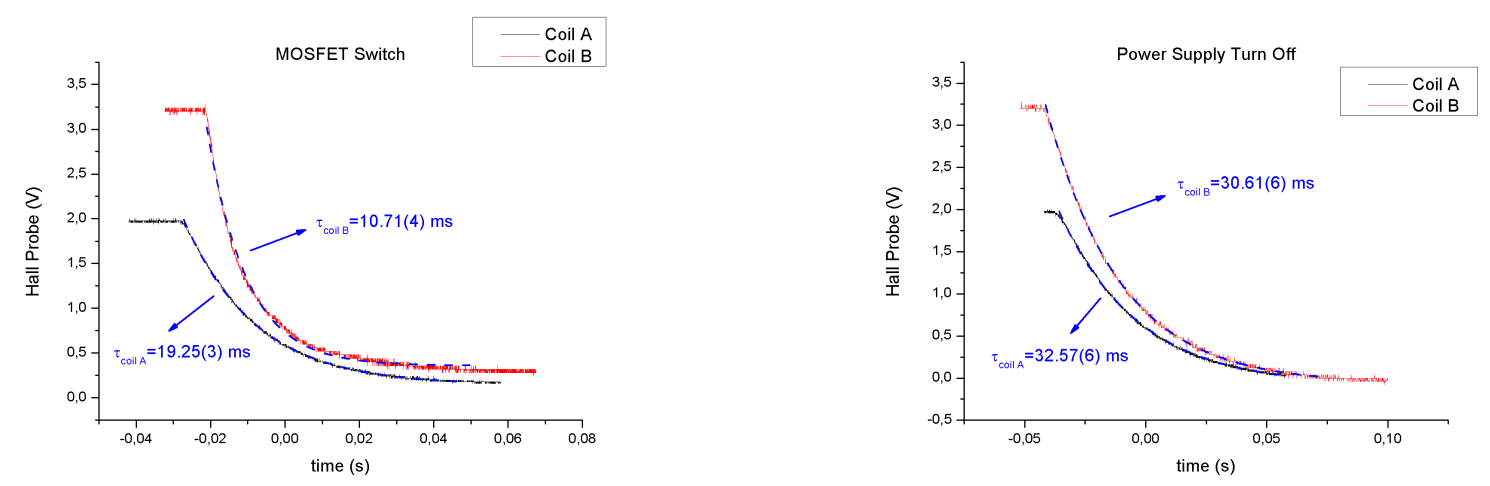

Figure 6 - Turning off the old MOT coils. Left: by using two MOSFET switches in reverse logic.

Right: by directly turning the power supply off.

Source: By the author.

By facing the problems listed above, we decided to design and construct a new generation of coils.

\subsubsection{New coils' project}

The new coils where designed using Mathematica package Radià. We only were constrained to the available power supplies. Different cooper wire diameters and profiles, 
position around the main chamber and sizes got considered. In the end, we choose a 2 $\mathrm{mm}$ thick wire wound in 11 lines x 18 columns resulting in 198 turns, resistance of $0.68 \Omega$ at $80^{\circ} \mathrm{C}$ and inductance of $7.3 \mathrm{mH}$.

In order to solve the overheat problem, we bought a chiller and decided to place the coil inside a closed structure made of non-metallic material (more precisely, polyacetal). This structure allows a direct contact between cooled water and the whole coil (as shown in Fig. 7). Moreover, the coils' turns are held together by a high thermal conductivity epoxi Duralco 132-2, to ensure that all the heat generated in its inner part could flow in the direction of the chilled water.

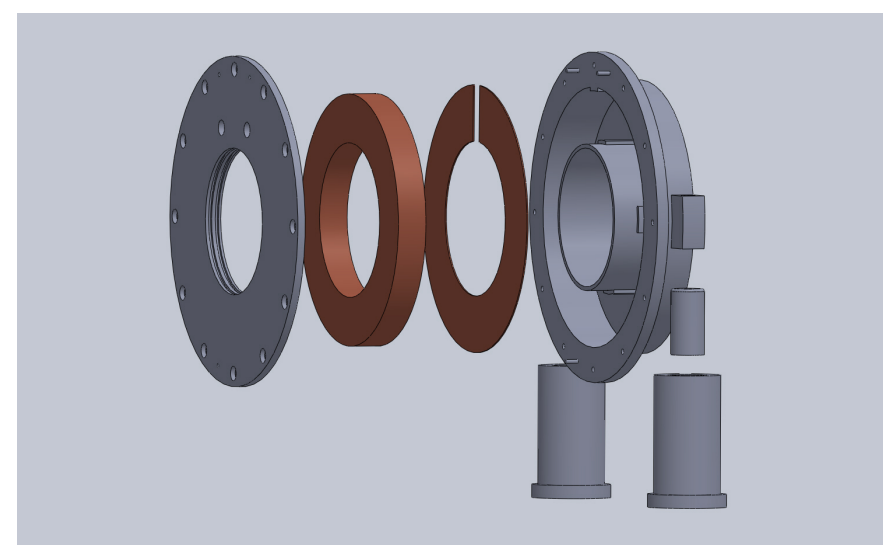

Figure 7 - Whole CAD project for the coil cooling housing.

Source: By the author.

\subsubsection{Construction and Characterization}

Both coils were wound in a designed support to have the right size and number of turns. As described above, we used a high thermal conductivity epoxi Duralco 132-2 to hold all the turns in place and a milling machine to help in the process of winding. Due to the wire packing, some deviations in the depth (smaller) and length (bigger) were observed.

After the epoxi got cured, we did the following characterizations: Resistance, Inductance, Magnetic field vs. applied current, Magnetic field along its axis, switch off time and Heat transfer to chilled water.

\section{Resistance}

The resistance were measured by applying a DC voltage in the coils terminals. The results for coil A and $\mathrm{B}$ are shown in Fig. 8, respectively. For coil A, the resistance is $R \approx 0.5 \Omega$, which corresponds to $N=203(3)$ turns. In coil $\mathrm{B}, R \approx 0.5 \Omega$, which corresponds to $N=201(2)$ turns. Both number of turns were calculated for a resistivity of $\rho_{C u}=1.70(2) 10^{-8} \Omega \mathrm{m}$, for a estimated temperature of $20-30{ }^{\circ} \mathrm{C}$. 

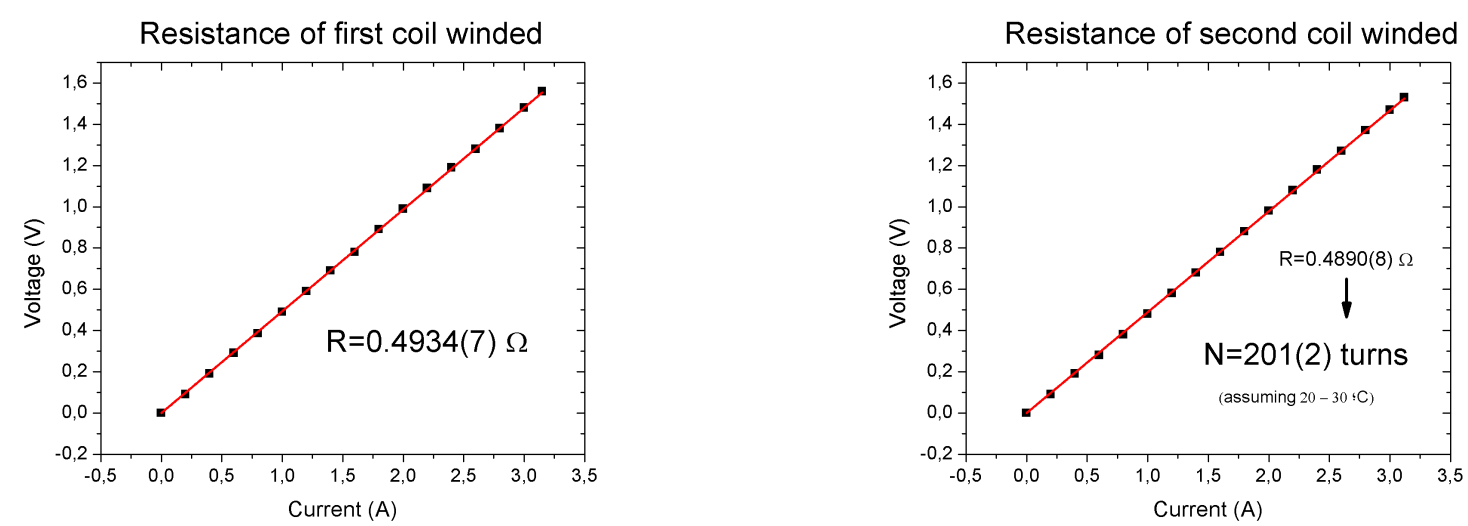

Figure 8 - Measurement of the resistance of the coils winded. Left: coil A. Right: coil B.

Source: By the author.

\section{Inductance}

In order to get the inductance, a standard RLC circuit (see Fig. 9) were used. This circuit is a series configuration of capacitance $C=2.2 \mu \mathrm{F}$, a resistor $R_{\text {probe }}=100 \Omega$ and the coil and it is supplied with an AC voltage (sinus waveform and $2 V_{p p}$ ). As the frequency, $f$, of the sinusoidal is changed, the voltage in the terminals of the resistor is measured resulting in a resonant curve $V_{\text {probe }}$ versus $f$. From the graph, it is possible to get the desired inductance.

The graphs obtained for coil A and B are shown in Figs. 10 and the inductance measured is $6.19(6) \mathrm{mH}$ and $6.30(7) \mathrm{mH}$, respectively. Both values are lower than the expected and a possible explanation to this deviation lies in the different packing of the wires.

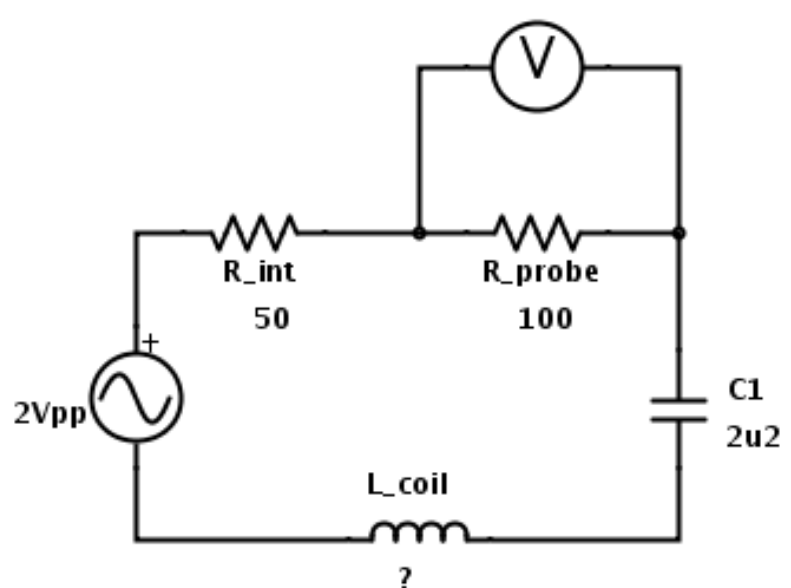

Figure 9 - Schematic RLC circuit used to measure the coil inductance. Source: By the author. 

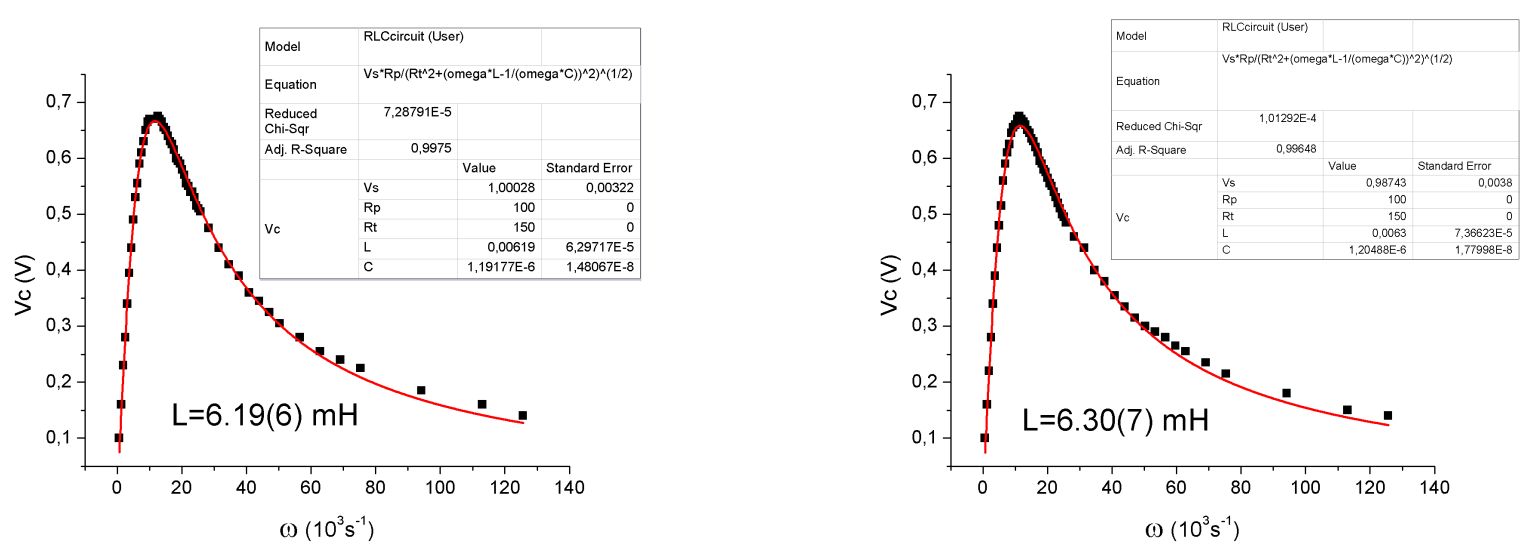

Figure 10 - Measurement of the inductance. Left: Coil A. Right: Coil B.

Source: By the author.

Magnetic field at the center of the coil

The expected magnetic field in the axis for a multi-layer solenoid is given by

$$
\begin{aligned}
B(z) & =\frac{\mu_{0}}{2} \frac{N}{L} \frac{I}{r_{1}+r_{2}}\left((z+L / 2) \ln \frac{\sqrt{r_{2}^{2}+(z+L / 2)^{2}}+r_{2}}{\sqrt{r_{1}^{2}+(z+L / 2)^{2}}+r_{1}}\right. \\
& \left.-(z-L / 2) \ln \frac{\sqrt{r_{2}^{2}+(z-L / 2)^{2}}+r_{2}}{\sqrt{r_{1}^{2}+(z-L / 2)^{2}}+r_{1}}\right),
\end{aligned}
$$

where $L$ is its length, $r_{1}$ and $r_{2}$ are the inner and outer radius, respectively.

The magnetic field in the center of the coil was measured scanning the applied current. The results are shown in Fig. 11. By using Eq. 3.1, the number of turns can be obtained: for coil A $N=203(1)$ turns and for coil B $N=203.5(1.1)$ turns.
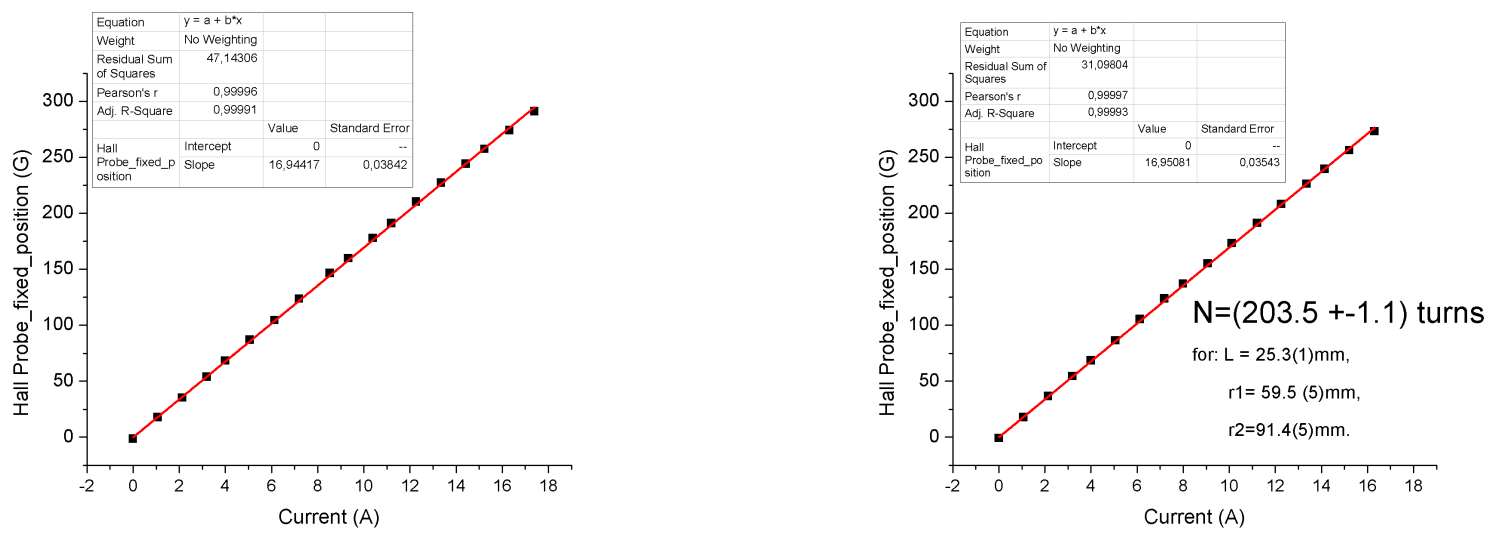

Figure 11 - Measurement of the magnetic field at the center of the coils. Left: Coil A. Right: Coil B.

Source: By the author. 


\section{Magnetic field along the axis of the coil}

A measurement along the axis of the coils were performed as well and are shown if Fig. 12. By using Eq. 3.1 a fit can be done around the center to obtain the number of turns, as the cited equation did not take in account the finite size of the coil. For coil A, $N=201.4(2)$ turns. For coil B, the fit did not converge.
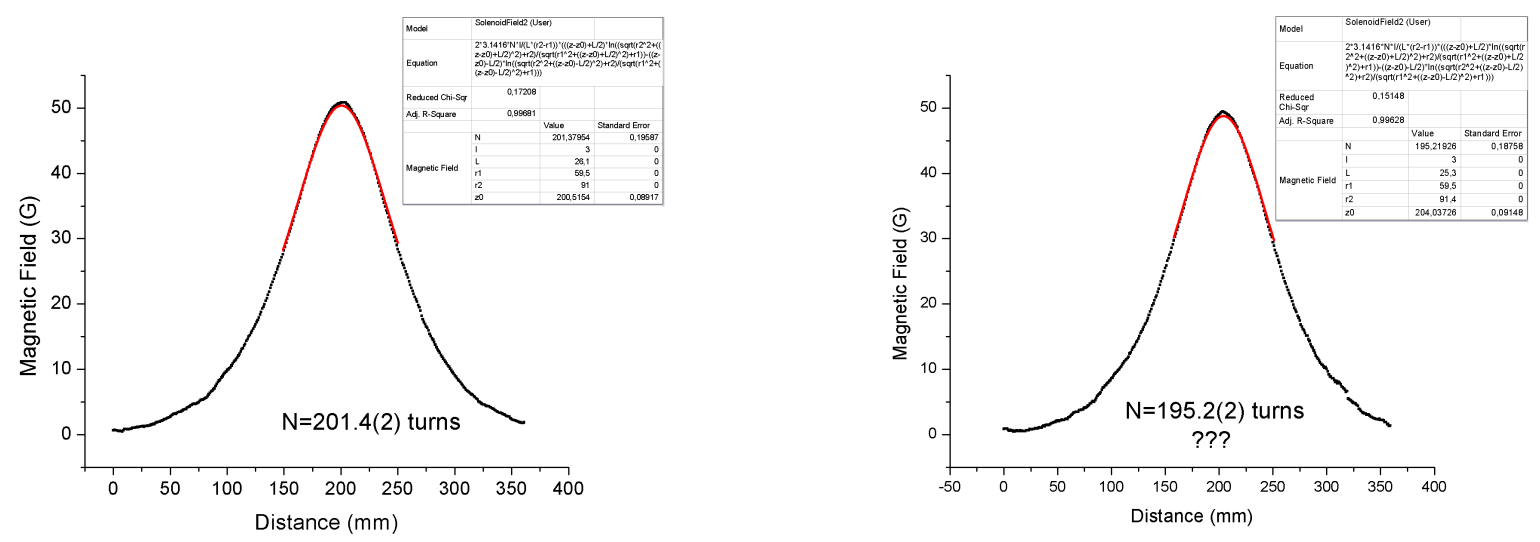

Figure 12 - Measurement of the magnetic field along the main axis of the coils. Left: Coil A. Right: Coil B.

Source: By the author.

\section{Magnetic field fall time}

Fig. 13) represents the magnetic field fall time for both coils. It was measured by using a Hall probe when a current of $30 \mathrm{~A}$ were applied to the coil. To control the current in the coil and switch it off quickly, an digital signal was sent to an mosfet. The time obtained is $\approx \mathbf{1 . 9 m s}$, which is at least 10 times better than the previous configuration. Unfortunately, as it will be pointed in the next chapter, this switch off time is still long. A good value should be around $100 \mu$ s. Further investigations are being performed but no positive answer have been reached.

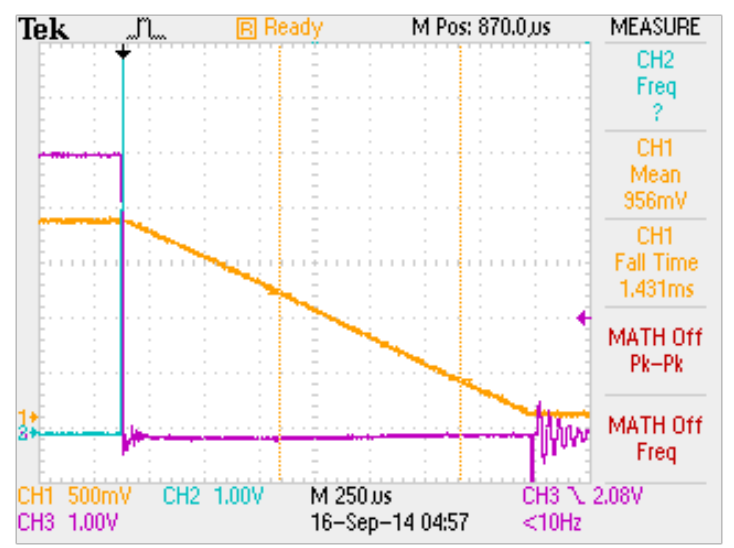

Figure 13 - Turning off response of first coil. Ch1: Hall probe signal; Ch2 and Ch3: controls for MOSFET switch.

Source: By the author. 


\section{Coils temperature and active dissipation}

In order the heat dissipation efficiency, a measurement of the temperature evolution of the coil were performed for currents between 5 and $40 \mathrm{~A}$ (maximum value allowed by the Agilent N5744A power supply). Thermocouples were installed inside the housing but it could only measure the chilled water temperature. However, the temperature can be calculated using the material resistance variation, due to Joule Effect, when a current is applied to it. This formula is

$$
R(T)=R_{T_{\text {room }}}\left[1+\alpha\left(T-T_{\text {room }}\right)\right]=R_{20^{\circ} \mathrm{C}}\left[1+0.00386\left(T-20^{\circ} C\right)\right],
$$

where $\alpha=0.00386$ is the temperature coefficient for copper and $T_{\text {room }}=10{ }^{\circ} \mathrm{C}$ is the room temperature.

Fig. 14 shows the results for this measurement. For them it is possible to notice that the coils reach a maximum temperature of $50^{\circ} \mathrm{C}$, which represents a better dissipation (the previous coils could reach $100^{\circ} \mathrm{C}$ before a steady state).
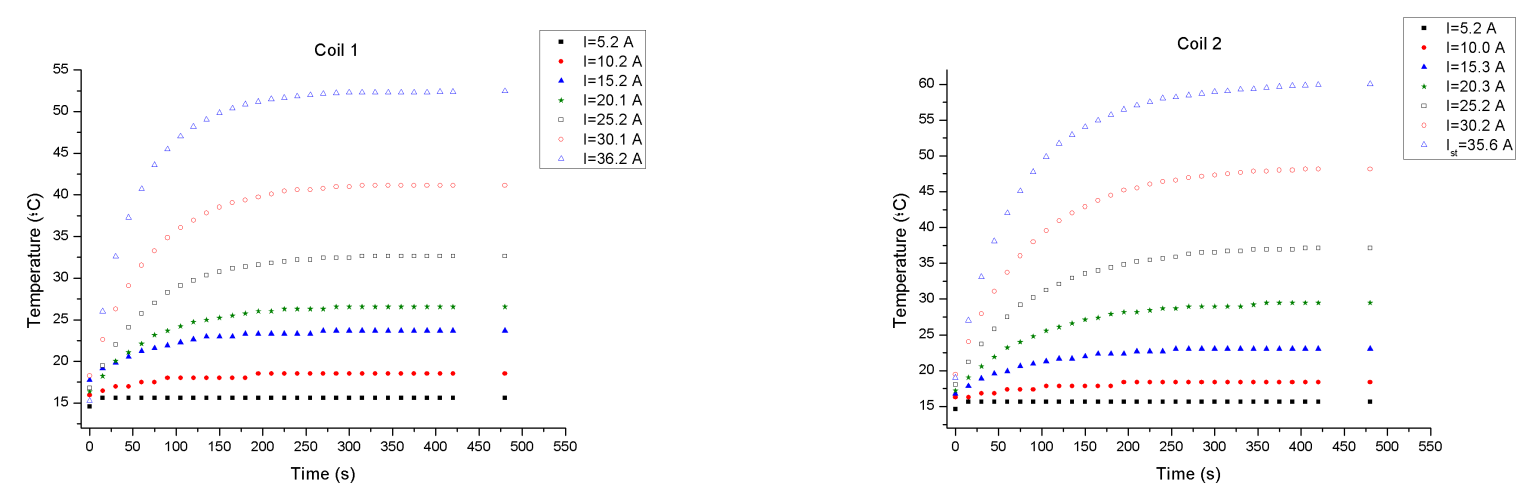

Figure 14 - Temperature evolution of the coils subjected to different currents (chiller flow on). Left: Coil A. Right: Coil B.

Source: By the author.

\section{Quadrupole magnetic field profile}

A measurement of the magnetic field profile of the coils when connected in antiHelmholtz and their distance was similar to what would be used around the main chamber was performed. The current applied to the coils was $3 \mathrm{~A}$ and the results is shown in Fig. 15. The gradient in the center of the configuration is $7.13(3) \mathrm{G} / \mathrm{cm}$, which means it is possible to reach the desirable value of $70 \mathrm{G} / \mathrm{cm}$ by using a current equal to $30 \mathrm{~A}$.

Then, it is possible to conclude that we have designed and constructed an appropriate pair of coils for our experiment, once we corrected the drawbacks presented in the beginning of this section. In Table 3, we present a summary of the coils characterization. 


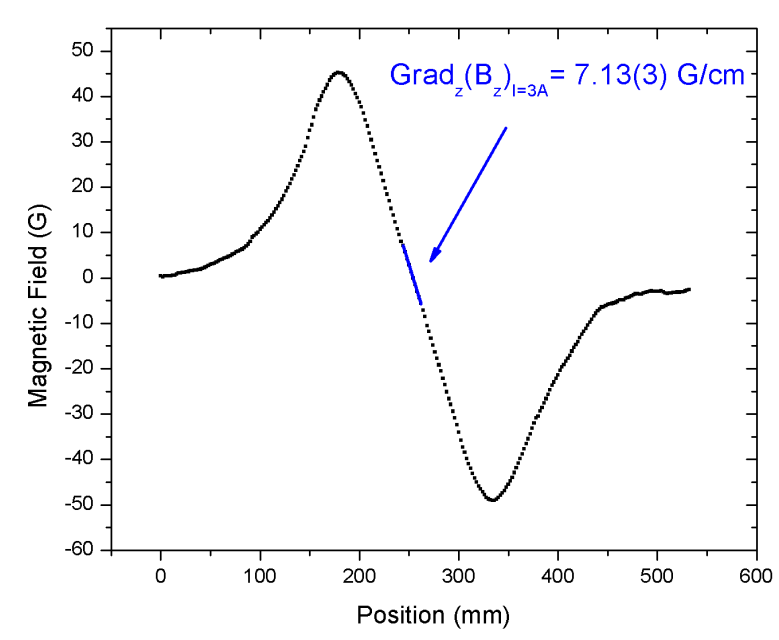

Figure 15 - Measurement of the quadrupole magnetic field along the main axis with $I=3 \mathrm{~A}$.

Source: By the author.

Table 3 - Summary of coils characteristics.

\begin{tabular}{l|c|c|c|}
\hline & Coil 1 & Coil 2 & Quadrupole \\
\hline \hline Length (mm) & $26.1(1)$ & $25.3(1)$ & \\
Depth (mm) & $33.5(5)$ & $33.9(5)$ & \\
Internal radius(mm) & $58.5(5)$ & $58.5(5)$ & \\
Resistance $(\Omega)$ & $0.4934(7)$ & $0.489(1)$ & \\
Inductance (mH) & $6.19(6)$ & $6.30(7)$ & \\
$N_{\text {turns }}$ - resistance & $203(3)$ & $201(2)$ & \\
$N_{\text {turns }}$ - Field vs Current & $203(1)$ & $203.5(1.1)$ & \\
$N_{\text {turns }}$ - Field vs Position & $201.4(2)$ & $195.2(2)$ & \\
Turning off time (ms) & $1.9(1)$ & $1.9(1)$ & \multirow{2}{*}{$7.13(3)$} \\
Gradient $(\mathrm{G} / \mathrm{cm}$ for 3 A) & & & \\
\hline
\end{tabular}

Source: By the author.

\subsection{Current and magnetic field control}

\subsubsection{Field effect transistor (FET)}

One of the most commom method used to control current flowing in coils is done by using Field Effect Transistor (FET), which is composed by semiconductor materials. A voltage applied in the gate controls the how much current is able to flow between the drain and source. We use an n-channel MOSFET (Semikron SKM-111AR) with a robust current rating for this experiment.

Fig. 16 shows the analog servo circuit where the FET is used. This servo circuit is an feedback loop and it is supposed to control and stabilize both current and magnetic field, avoiding any oscillations or spikes in order to protect the coil, its components and the power supply. Another important point is that any fluctuation in the magnetic field is transferred to the magneto-optical trap, which could heat up our samples. The servo 


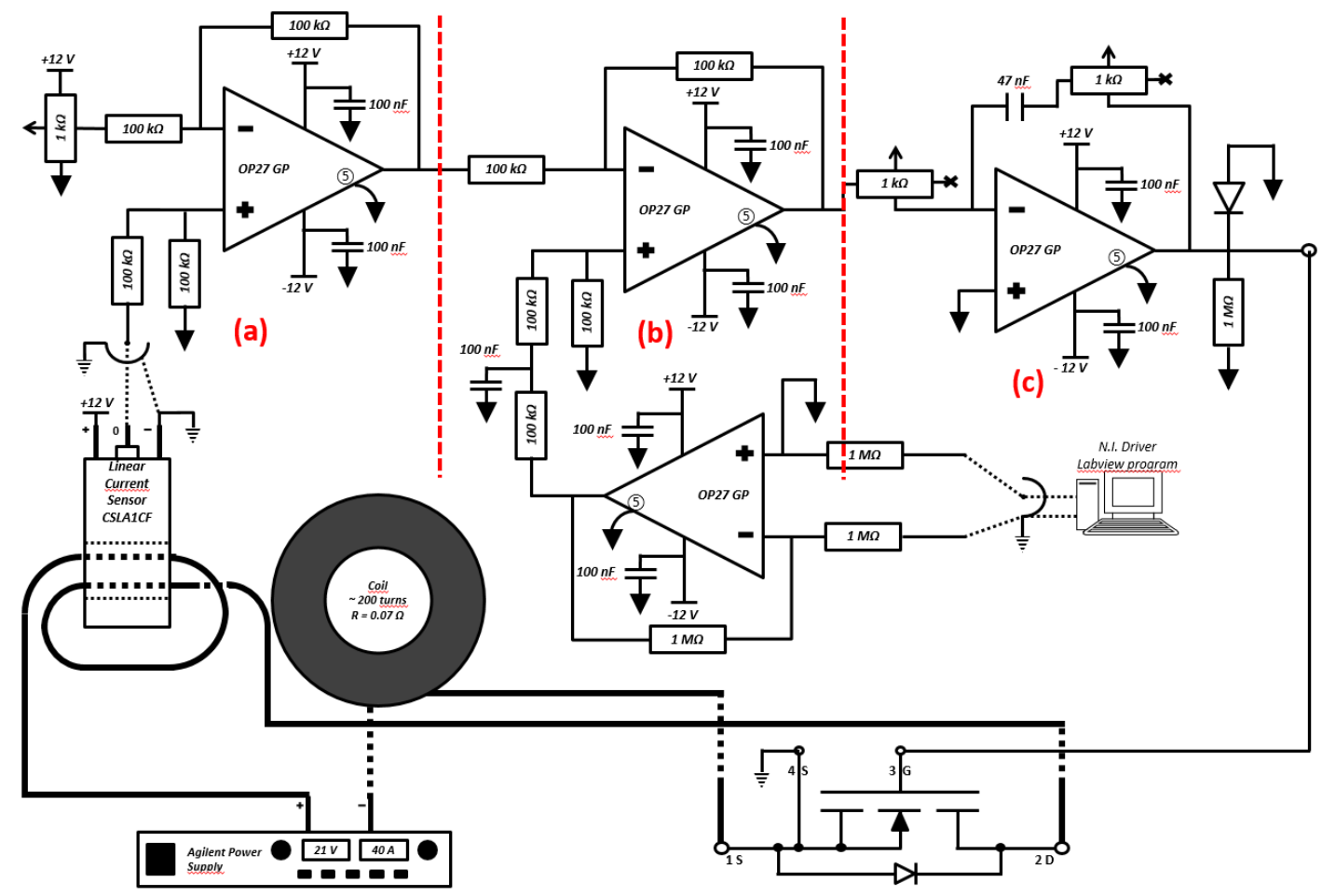

Figure 16 - Schematic diagram of the feedback loop. (a) Linear current sensor offset voltage removal part; (b) Operational amplifier comparator stage: computer control signal get compared to control fluctuations and allow current changes;and (c) PID circuit.

Source: By the author.

compensates any deviation in the desired magnetic field by comparing it with what is being measured by an transducer. This comparison creates an error signal which is sent to a proportional integral derivative (PID) controller which adjusts the current via a variable analog signal. The coil current is supplied by an Agilent N5744A power supply, connected between the drain and the source of the FET.

The circuit works as follows: a voltage analog signal (created by our experimental control software) controls the gradient of the magnetic field in the magneto optical trap. The current flowing through the coil is measured by an linear current sensor (Honeywell CSLA1CE). Both signals are compared and the result voltage is sent to an PID controller which is connected between gate and source of the FET, resulting in a signal which correct any fluctuation in the magnetic field. Any sudden variations will be smoothed out without influences in the characteristic switch on an off times of the coil.

\subsection{Blue Laser systems}

The light that drives the transition ${ }^{1} S_{0} \rightarrow{ }^{1} P_{1}$ is provided by a frequency-doubled diode laser bought from Toptica Photonics. It consists of a diode laser in Littrow configuration running at $\lambda=922 \mathrm{~nm}$ that emits $50 \mathrm{~mW}$ at a typical current of $130 \mathrm{~mA}$. This light is used to seed a tapered amplifier (TA) to roughly $0.95 \mathrm{~W}$ behind the isolator. The infrared light is put inside a resonant cavity where it is sent through a crystal for frequency doubling, resulting in a output power of $360 \mathrm{~mW}$ at $\lambda=460 \mathrm{~nm}$. 


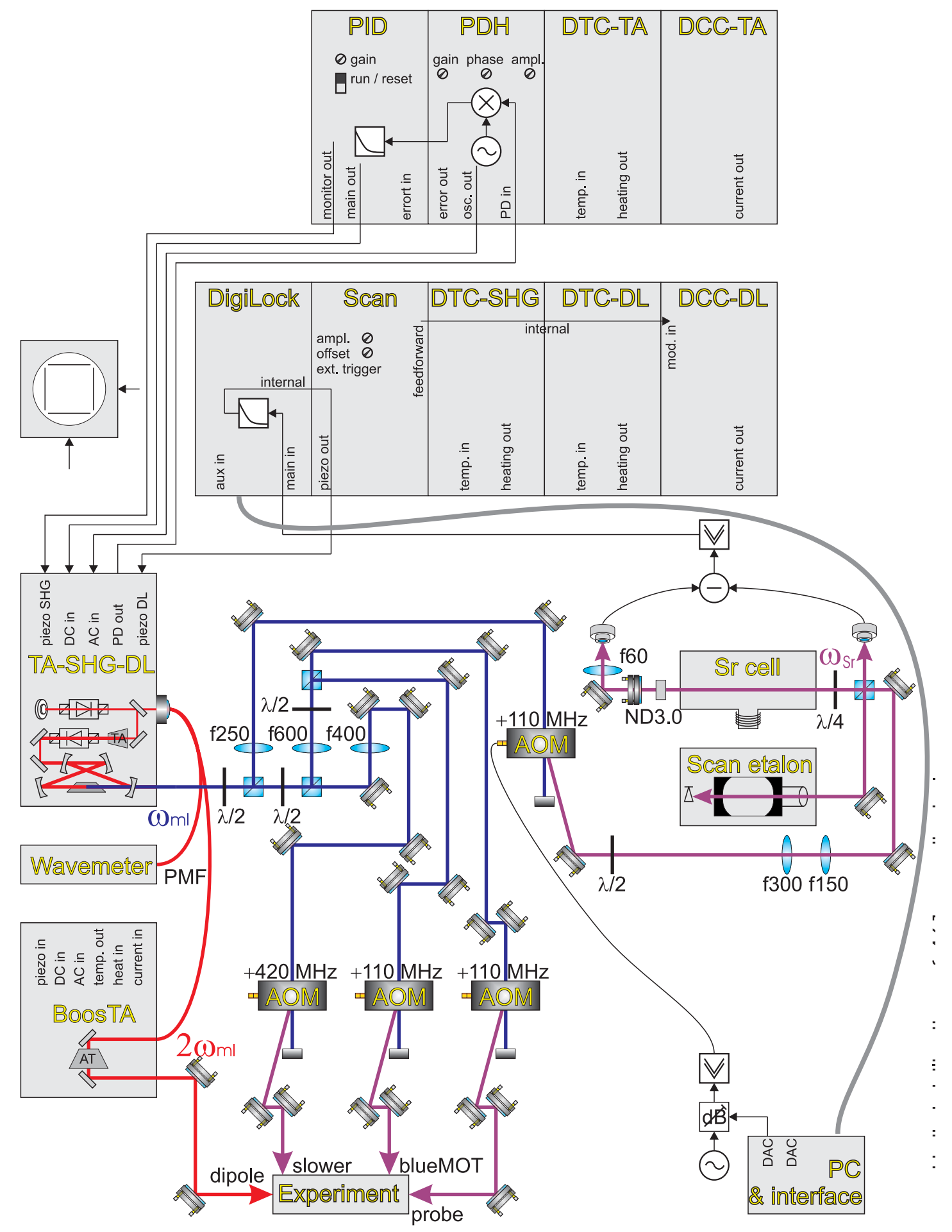

Figure 17 - Schematic representation of the blue laser system, more details in the text.

Source: By the author. 
The laser beam is then divided, by polarization optics, to be used in all the different parts of the experiment. Fig.18 shows our current blue laser system. Each of these beams passes once through an acousto-optical modulator (AOM) to shift the light frequency to the desired value and for fast power-switching of the beam. The laser runs at $\nu_{\text {laser }}=$ $\nu_{0}-130 \mathrm{MHz}$, where $\nu_{0}=650.5037 \mathrm{THz}^{*}$ is the transition frequency ${ }^{1} S_{0} \rightarrow{ }^{1} P_{1}$. In the following subsections all the different parts of the blue laser system will be described (a schematic version of the blue system is represented at Fig. 17.).

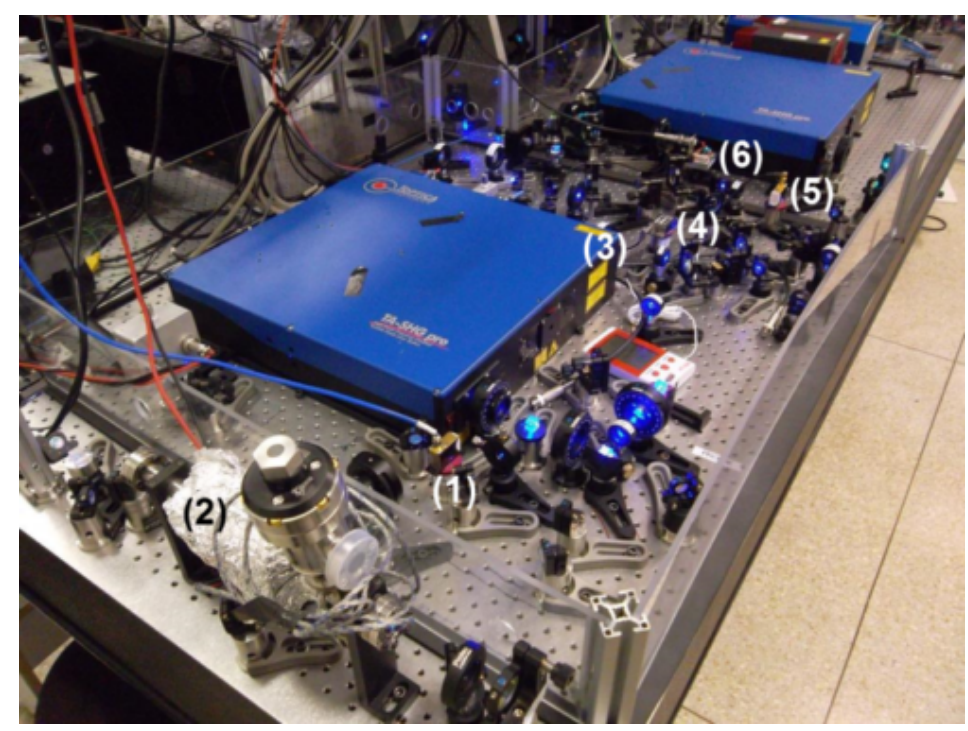

Figure 18 - The blue (center) and green (top right side) laser system. For the blue laser: (1) represents the spectroscopy AOM, (2) the spectroscopy cell, (3) the Zeeman slower beam AOM, (4) the MOT beams AOM, (5) the absorption imaging beam AOM and (6) the green beam AOM.

Source: By the author.

\section{Doppler-free saturation spectroscopy}

In order to stabilize the laser at the desired frequency, it needs to be locked to an external frequency reference via an electronic feedback. In our experiment, the feedback is provided by the commercial Toptica DigiLock system. The frequency reference is obtained via standard Doppler-free saturation spectroscopy by shining a $10 \mathrm{~mW}$ of blue light, whose frequency is shifted up by $130 \mathrm{MHz}$ by an AOM onto a spectroscopy cell which contains strontium at a temperature of $300^{\circ} \mathrm{C}$. Fig. 20 represents a typical spectroscopy signal (more details in 3.6).

\section{Zeeman slower beam}

A part of the light is used to decelerate the fast atomic beam leaving the oven to velocities that can be captured inside the MOT. The current Zeeman slower uses light at a frequency of $\nu_{\text {Zeeman }}=\nu_{0}-560 \mathrm{MHz}$ and a power of $70 \mathrm{~mW}$. After passing the AOM the beam is widened to an initial $1 / e^{2}$ radius of $w_{0}=5 \mathrm{~mm}$ and them slightly focused after

\footnotetext{
*This value was measured by using a High Finesse WSU 30 wavemeter.
} 
$2 \mathrm{~m}$ in order to fit the microtubes holder aperture $(2.1 \mathrm{~mm}$ diameter $)$ and compensate for the absorption of photons in the slowing process, increasing the intensity on the ZS beam. The beam is adjusted counter propagating to the atomic beam leaving the oven.

\section{MOT beams}

The frequency in the MOT beams is $\nu_{M O T}=\nu_{0}-40 \mathrm{MHz}$. After the AOM the MOT beam passes a telescope to form a collimated beam with a waist of $w_{0}=11 \mathrm{~mm}$. The light is separated in three further beams that are shined perpendicularly to each other in the axial and radial directions of the chamber, with an optical power of $4.5 \mathrm{~mW}$ and 8 $\mathrm{mW}$, respectively. Then, they pass through $\lambda / 4$ waveplates, to create circularly polarized light, and them to the science chamber. The beams are retro-reflected by a mirror in order to provide the three missing beams required to operate the MOT. Due to the absence of coating in the viewports of the chamber, there is a small loss of power in all retroreflected beams compared to the incoming ones.

\section{Absorption imaging beam}

In order to get information about the atomic clouds density distribution, atom number and temperature, we make use of the standard absorption imaging technique. A $40 \mathrm{~mm}$ focal length lens images the atoms to a CCD-Camera chip ${ }^{\dagger}$, while the atoms are uniformly illuminated by a $200 \mu \mathrm{W}$ collimated resonant laser beam with waist of 12 $\mathrm{mm}$. The CCD camera is placed after a system of lenses composed by one lens with focus $f=125 \mathrm{~mm}$ followed by another one with focus $f=100 \mathrm{~mm}$, which results in a collimated absorption imaging beam and a magnification of the MOT image equal to 0.8. Fig. 22 shows an example of the absorption imaging of our samples.

\subsubsection{Green laser}

We use a Toptica SHG system, an schematic version of this laser system can be found in Fig. 19, to generate the $497 \mathrm{~nm}$ light, Fig.18 shows our current green laser system. An IR diode at $994 \mathrm{~nm}$ generates about $300 \mathrm{~mW}$ of power and is monitored on a FPI and a wavemeter. The light is coupled into a SHGpro doubling cavity, which generates up to $45 \mathrm{~mW}$ of green light if optimized. This light is coupled into a fiber for mode-cleaning and ease of operation. One AOM with nominal frequencies of $80 \mathrm{MHz}$ in single-pass configuration is used for fast switching.

We use a green laser that is resonant to the $5 s 5 d^{3} D_{2}$ state, which can decay to ${ }^{3} P_{1}$, which in turn decays back to the ground state. It is difficult to set up a spectroscopy for this transition. It turned out however that once the frequency is set, the laser stays stable. The first frequency set is done by optimizing optically the brightness of the MOT.

\footnotetext{
${ }^{\dagger}$ Point of Grey Chamaleon USB whose pixel size is $3.75 \mu \mathrm{m} \times 3.75 \mu \mathrm{m}$
} 


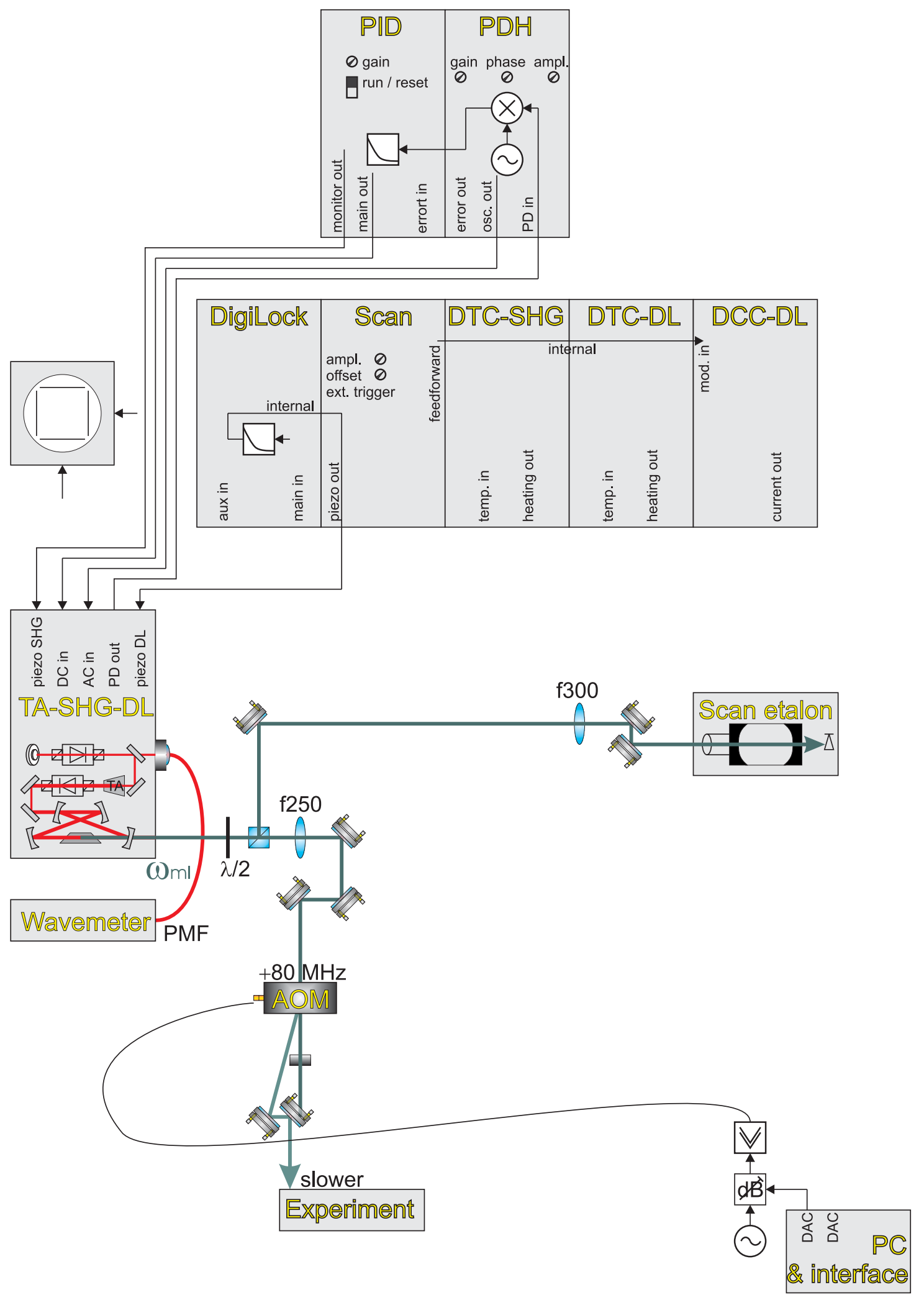

Figure 19 - Schematic representation of the green laser system.

Source: By the author. 


\subsection{Blue laser frequency stabilization}

The output frequency of a semi-conductor diode laser is in general not stable when free running. Comparing the frequency to an external reference allows for the creation of a feedback loop to stabilize the laser frequency. First an external reference is created by performing saturated absorption spectroscopy on the $\left(5 s^{2}\right)^{1} S_{0}-(5 s 5 p)^{1} P_{1}$ transition. The absorption spectrum is passed to a Toptica Digilock module. The frequency is then modulated to provide a lock-in derivative signal to which the frequency can be locked.

\subsubsection{Saturated Absorption Spectroscopy}

The optical system for the saturated absorption spectroscopy is part of the blue optical system shown in Fig. 17 is used to obtain a saturated absorption signal from a strontium gas cell, heated to $300{ }^{\circ} \mathrm{C}$ to obtain a high $\mathrm{Sr}$ partial pressure. The cell is filled up with buffer gas (Argon) until a pressure of 1 mbar is reached to avoid strontium atoms to impinge the windows. The input beam is first put through an AOM, with the first order being used, giving a frequency shift of $130 \mathrm{MHz}$. The beam is divided into three components using two polarizing beam splitters: the strong, probe and balance beams. The strong and probe beams are directed through the spectroscopy cell such that they are counter-propagating and nearly superimposed. After traveling through the cell the probe beam is directed onto a photodiode, where a simple subtraction is performed with the balance beam, thus removing variations in the master laser intensity. The balance beam is also split to provide an output for a scanning etalon for analyzing the mode structure of the laser.

The Doppler broadened absorption spectrum can then be observed as a function of frequency by scanning the piezo attached to the grating of the DL pro, i.e. giving some amplitude to the piezo scan on the SCC110 controller. The strong beam, having a much higher intensity than the probe, causes spectral hole burning by saturating the transition near resonance. Fig. 20 shows the transmitted intensity of the probe beam, after subtraction with the balance beam, with and without the presence of the strong beam and the zoom in the Lamb dip. The probe transmission shows an increased intensity near resonance, with a local maximum on resonance, when the strong beam is saturating the transition, a feature known as the Lamb dip. For the $\left(5 s^{2}\right)^{1} S_{0}-(5 s 5 p)^{1} P_{1}$ transition this occurs at a central frequency of $650.5032 \mathrm{THz}(650.5037 \mathrm{THz}$ as measured on High-finesse wavemeter WSU) and has a width of $30.8 \mathrm{MHz}$. The balanced probe signal is passed to the Digilock module to allow locking. In order to achieve good contrast, the strong beam should be between 10 and 50 times stronger than the probe beam.

In the Fig. 20, the measurement of the dip profile reveals an FWHM of 44(1) MHz. Since the natural linewidth of the blue transition is $30.8 \mathrm{MHz}$, this broadening can be only explained by collisional broadening due to interactions between strontium and the buffer gas, and other strontium atoms. The broadening for both events cited are estimated to be $8.8 \mathrm{MHz}$ and $2.8 \mathrm{MHz}$, respectively. Then, the broadened linewidth is equal to $42(2)$ $\mathrm{MHz}$, confirming the value obtained by our measurements.

Steps to align the system:

1. Align beam into first order of the AOM, check that the AOM frequency is at 130 

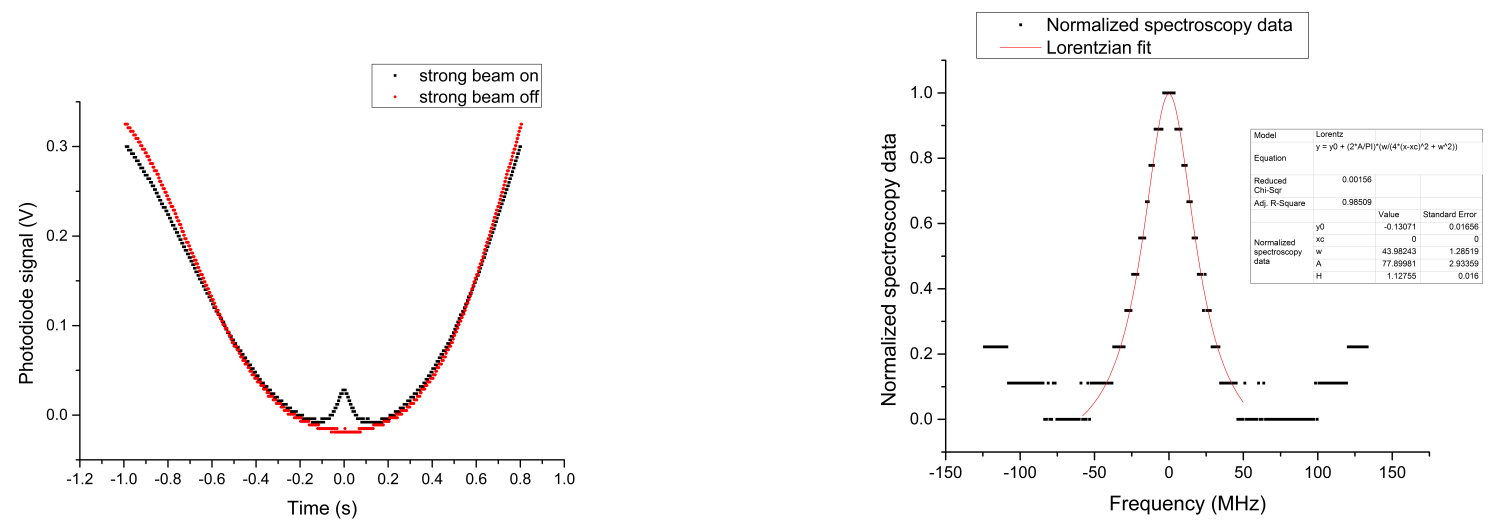

Figure 20 - Absorption spectrum. Left: with the strong saturating beam on (black), where a Lamb dip is exhibited, and off (red); by optimizing the overlap and power ratio of the strong-probe beams allows Lamb dips of order $100 \mathrm{mV}$. Right: Zoom on the Lamb dip obtained in our saturated absorption imaging apparatus. After fitting, the half width at half maximum is equal to 44(1) MHz.

Source: By the author.

$\mathrm{MHz}$ before continuing. Ensure that the beam is at correct beam height and travels through center of first lens;

2. Align the beam in such a way that it passes through center of second lens. Align both cubes, strong-weak and probe-balance, in such a way that the beam is well centered and their reflected ports are perpendicular to input beam;

3. Align the balance beam onto photodiode, the probe beam onto the entrance port such that it travels towards photodiode and strong beam onto the opposite port;

4. Align the weak beam through cell and onto photodiode. Final cube is needed to separate probe beam from counter-propagating strong beam;

5. Adjust laser frequency (check it with a wavelength meter), scan amplitude and the beam powers until absorption line can be observed on the output from photodiodes;

6. Superimpose the strong beam with the probe while looking for the Lamb dip on the photodiode signal. Ensure the probe beam remains on the photodiode using the last cube and the mirror if using mirrors which influence both beams;

7. Loosen the clamp holding the cell to the table and slightly move the cell until both beams have good shape after travelling through chamber.

Once you have a Lamb dip and a good beam shape, it is easier to optimize by beam-walking the probe over the strong beam. Also optimize power balance between strong-probe and probe-balance. Try to ensure no strong beam intensity is impinging on the probe photodiode - this will reduce the observed dip. For this reason the best dip may not be when the beams are perfectly superimposed, but rather slightly misaligned. 


\section{Comparison between locked and free running laser}

Fig. 49 shows the frequency versus time for the locked laser, free running and free running with Digilock module switched off measured using the High Finesse accurate wavelength meter, while Fig. 21 shows the same image expanded in the frequency axis. This shows that when free running the laser drifts by approximately $5.2 \mathrm{MHz}$ per minute compared with no clear drift for the locked laser. Analysis of the frequency over short terms is not relevant without changing the input fiber to single mode. Also it is worth noting that while the spectroscopy is stabilized against intensity fluctuations, the wavelength meter is not - meaning intensity noise is influencing the frequency accuracy.

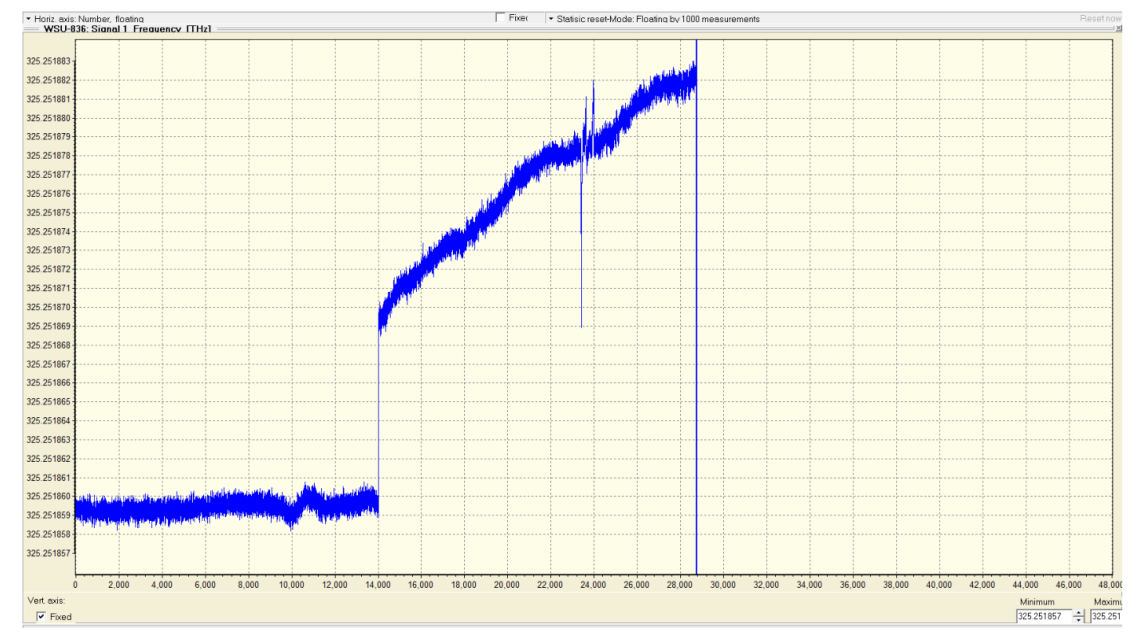

Figure 21 - Close up of Fig. 49 showing frequency versus time for the locked and free running laser. One minute corresponds to 6000 measurements. Measured using High-finesse WSU wavelength meter.

Source: By the author.

\subsection{Blue MOT}

After aligning and optimizing all parameters of the blue and green laser systems, we performed the experimental sequence to trap the atoms in a MOT via ${ }^{1} S_{0} \rightarrow{ }^{1} P_{1}$. Fig.2 shows a temporal evolution of the experimental sequence in this stage and for the recapture in the narrow transition. All the digital and analog signals used in our experiment for fast switching of AOMs, control of magnetic fields and control of the CCD camera are generated by National Instruments (NI) PCI cards. These signals can be controlled by a Labview interface, where an pre-defined experimental sequence written in Python can be choosen. The output of this source code is read by Labview and send all the data from the experimental sequence to the NI PCI cards.

In the current generation of our experiment, we are able to obtain a maximum of $3 \times 10^{8}$ atoms trapped in the blue transition at a temperature of few $\mathrm{mK}$, when the repumper is used (see Fig. 22 for an example). The MOT lifetime is $2 \mathrm{~ms}$. After this stage, the atoms in this trap can be used in experiments but even more cold samples can be obtained. In the next chapter, the transfer of atoms in the blue MOT to the narrow red transition will be explained. 


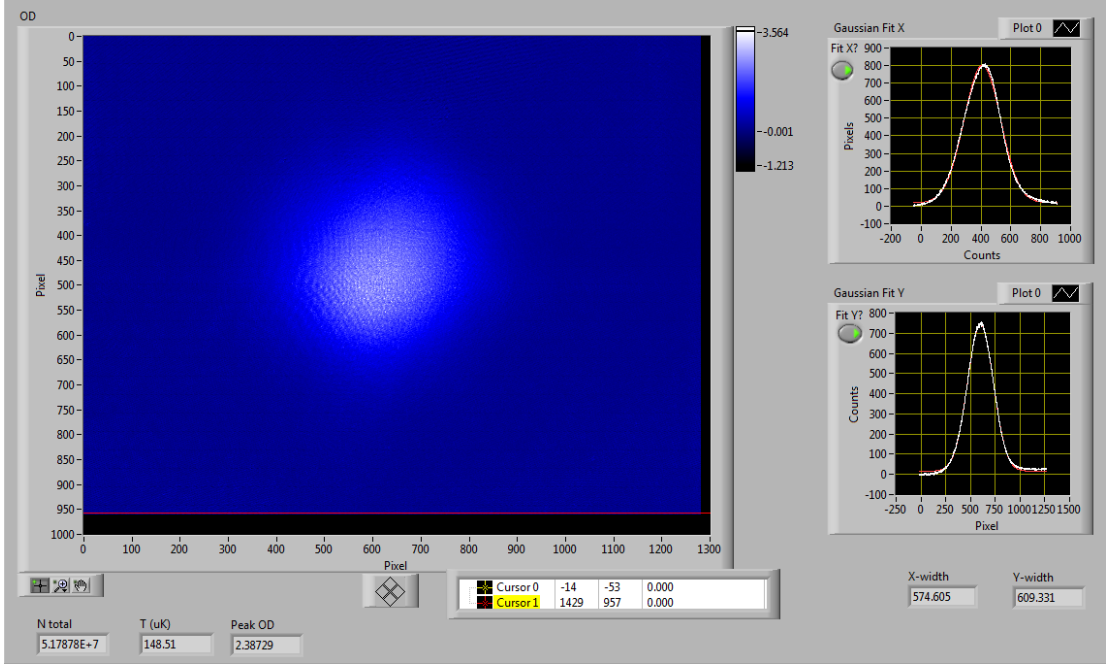

Figure 22 - Absorption imaging picture and analysis of our cold Sr samples. Here $N_{\text {atoms }}=$ $5 \times 10^{7}$ atoms and a temperature $T \cong 5 \mathrm{mK}$.

Source: By the author. 



\section{Red laser system}

\subsection{Ultra-stable Fabry-Pérot cavities}

Fabry-Pérot optical cavities has a huge number of applications in science. In atomic physics, they are used to stabilize lasers and reduce their linewidth so it can be used to probe narrow atomic transitions and as monitor of the modes of a laser. In this section, some concepts of this important device will be presented.

\subsubsection{Fabry-Pérot Optical Cavity Theory}

A conventional Fabry-Pérot etalon is an optical cavity and it consists of two parallel reflective mirrors set a certain distance apart from each other. This distance is critical and usually a spacer, that can be made out of different materials (e.g., low thermal expansion material and aluminum), is used to maintain it fixed. A study in different configurations of FP cavities and its stability can be found in (26).

An optical cavity can be understood as an interferometer. In this scenario, light enters the apparatus by one of the mirror (incoupling mirror) and it is reflected back from the second (outcoupling) mirror, process which is repeated indefinitely. Inside the cavity, constructive and destructive interference patterns are created, depending on the modematch between wavelength and cavity length, which controls the phase between the incoming and reflected light.

The first important parameter for a FP cavity is the free spectral range (FSR), which is defined as the frequency separation between two consecutive modes that will be transmitted through the optical cavity; an example is shown in Fig. 23. This frequency separation between resonant modes is also defined as $\nu_{F S R}=c / 2 L$, where $\mathrm{c}$ is the speed of light.

The linewidth of the resonances is also important and is determined by the reflectivity of the mirrors. For a low reflectivity each photon will bounce back and forth a smaller number of times, thus reducing the quantity of optical paths to interfere constructively, which results in a wide range of wavelengths will be transmitted. On the other hand, for a high reflectivity of the mirrors, the constructive interference will be more selective which results in narrower resonant modes. In the cavity spectrum, the linewidth $\Delta \nu$ is defined as the full width half-maximum (FWHM) of the resonant peak.

From the above definitions, one can define the finesse $F$ as the ratio between the free spectral range and the linewidth. The higher the finesse, the narrower the resonant modes and the higher resolution frequency discrimination we have for light passing through the optical cavity. For mirrors with a high reflectivity $R$, where absorption or losses can be negligible, the finesse is mathematically defined as

$$
\mathcal{F}=\frac{\nu_{F S R}}{\Delta \nu} \approx \frac{\pi \sqrt{R}}{1-R}
$$




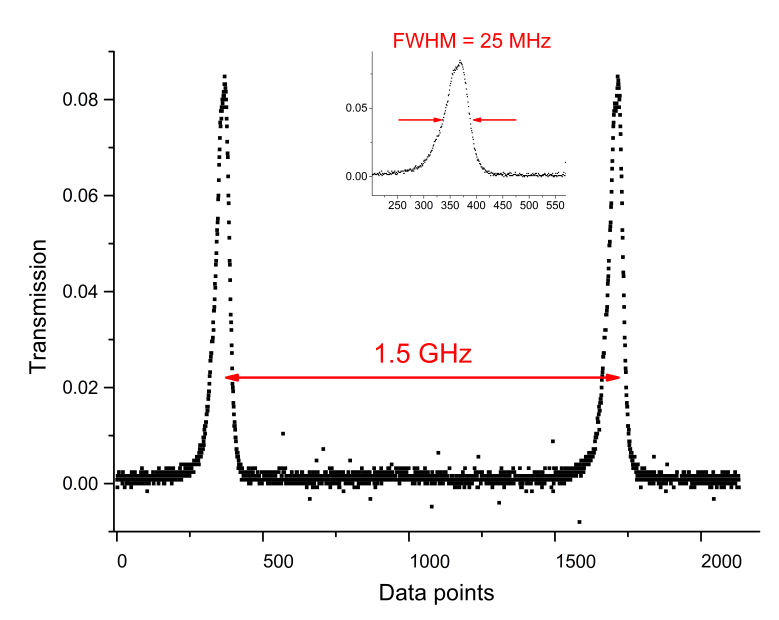

Figure 23 - The transmission spectrum of a Fabry Pérot Cavity with a finesse of 60 . The measurement was done by changing the length of the homemade optical cavity described in Table 4. The cavity length was changed by having one of the mirrors mounted on a piezoelectric transducer and changing the voltage supplied. The data points are an arbitrary measure of the linear change in length of the cavity. The insert is a magnified image of the transmission peak. The asymmetry observed in the shape of the linewidth is believed to be due to higher order modes being transmitted by the optical cavity.

Source: By the author.

In our experiment, an optical cavity is used to reduce the frequency variations and therefore reduce the linewidth of our laser frequency by electronic feedback to the laser current and grating angle control.

Table 4 - Measured specifications of our Fabry-Pérot cavities.

\begin{tabular}{ccc}
\hline & Homemade cavity & UltraStable Systems cavity \\
\hline \hline Arrangement (Curvatures) & Confocal & Non-confocal \\
Optical Cavity Length & $5 \mathrm{~cm}$ & $50 \mathrm{~cm}$ \\
Free spectral range, $\nu_{F S R}$ & $1.5 \mathrm{GHz}$ & $1.5 \mathrm{GHz}$ \\
Finesse, $\mathcal{F}$ & 60 & 7000 \\
Linewidth, $\Delta \nu$ & $26 \mathrm{MHz}$ & $215 \mathrm{kHz}$ \\
\hline
\end{tabular}

Source: By the author.

\subsection{Homemade scanning confocal etalon}

The theory of a Fabry-Pérot theory is described in sections 4.1 and 4.1.1. Among all the different kinds of FP cavities, the confocal is one of the most used configuration due to its potential uses in spectroscopy, frequency markers and characterization of the laser spectrum. This device is composed, as the name says, by two identical spherical mirrors separeted by a distance equals to two times their focal length, or one time its radius of curvature. 
In our experiment, a confocal FP is widely used to spectrally characterize our lasers, in order to maintain them in a single-mode regime (for all wavelengths), and as a checkpoint for the injection lock performed in the red laser system. The device is modular and can be constructed by any group with a good mechanical workshop, with different finesses and purposes.

The whole assembly (see Fig. 24) is made of aluminum and it is composed by a central hollow cylinder shape body (see Fig. 24(g)), which is used only as the spacer, and disks which are used to hold the mirrors (see Fig. 24(f) and (h)), hollow cylinder shaped piezo (see Fig. 24(e)) and photo-diode (see Fig. 24(b)) in place.These disks are connected to the main body and to each other by screws. Furthermore, a laser beam can be aligned through its axis and all the other parts can be assembled later. The components we use to build up our etalons are: piezo-electric is HPSt 150/14-10/12 (American Piezo Ceramics), photodiode is the Thorlabs FDS-100 and the mirrors are made by our Optics Workshop.
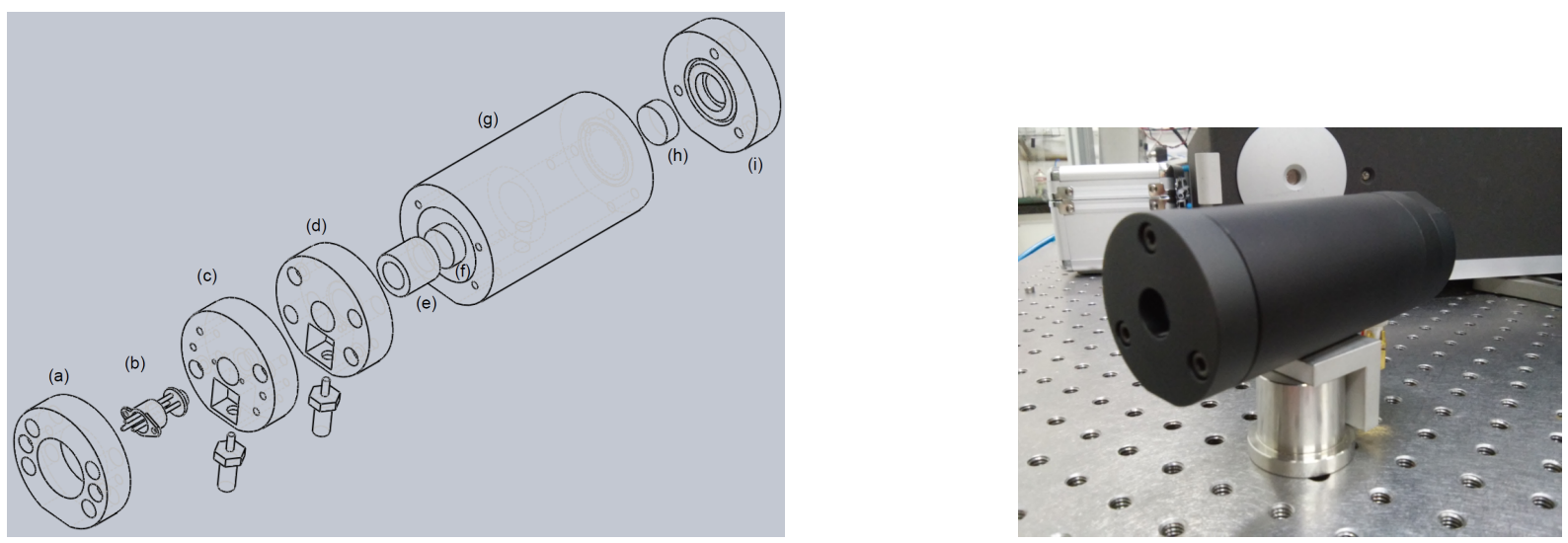

Figure 24 - Homemade scanning etalon. Left: CAD representation of the homemade scanning etalon (a)End part disk; (b) photodiode and its holder; (c) Photodiode disk; (d) Outcoupling piezo/mirror disk; (e) Hollow cylinder shaped piezo; (f) Outcoupling spherical mirror; (g) Main body/spacer; (h) Incoupling mirror; and (i) Incoupling mirror disk. Right: Picture of the final product.

Source: By the author.

The finesse of this homemade finesse will depend only on the reflectivity of the mirrors used and/or the ability of the supplier hired. In our experiment, he use a $5 \mathrm{~cm}$ long confocal FP cavity and we are able to get finesses between 100 and 400 (which means $R=96.9 \%$ and $R=99.3 \%$, respectively), but there is no limit for its number (Fig. 25).

\subsection{Laser Locking to an Optical Cavity}

Our aim is to improve the stability of our laser by locking it to an optical cavity resonance. In this case, these resonances are used to give electronic feedback to the laser frequency control to them electronically reduce the linewidth using a closed-loop servo system. The bandwidth of our locking electronics should be controlled because it is linked to the range of frequencies the system is able to correct for.

To lock the laser to the optical cavity, it is necessary to maintain a maximum transmitted intensity of the light through the optical cavity, by setting the laser fre- 


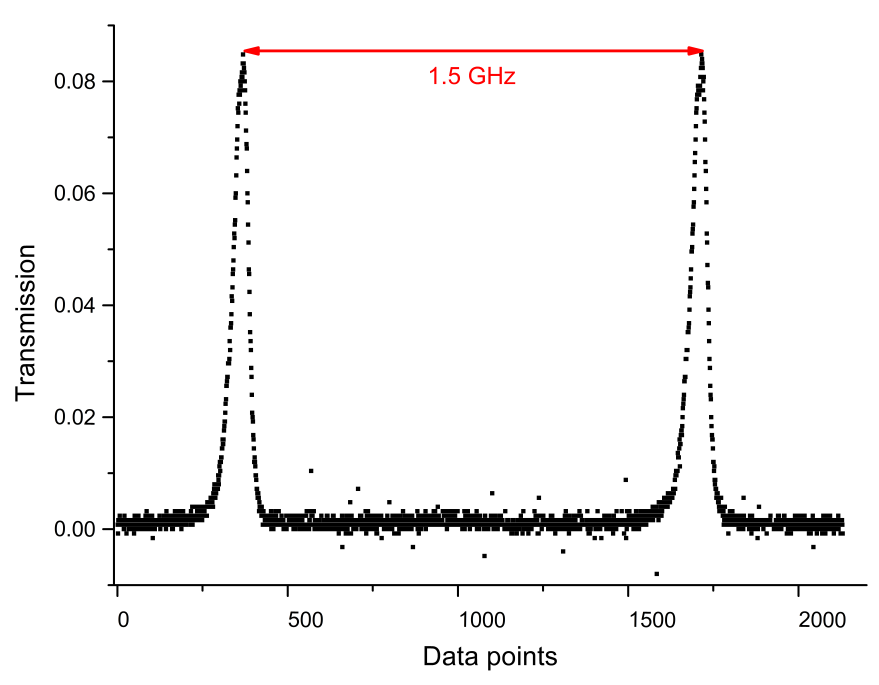

Figure 25 - One free spectral range a confocal FP coupled to the red laser system.

Source: By the author.

quency. However, when it moves off resonance the reduction in transmitted intensity can be measured but there will be an uncertainty in the direction in which the laser frequency should be corrected. Other possibilities in locking to a maximum of intensity can be exploited but they will result in the same problem.

\subsection{Pound-Drever-Hall (PDH) Locking}

To overcome any problems faced when the maximum transmitted intensity in a optical cavity is used, the Pound-Drever-Hall locking (PDH) (27) can be used. To employ this technique, sidebands needs to be created in the laser light, either by frequency or phase modulation, in order to create an error signal.

In more details, when the laser beam with its carrier and sidebands is incident on an optical cavity the reflected sidebands and the carrier will experience a phase change, depending on their relative frequency distance from the closest resonance. By detecting the reflected light from the cavity with a photodiode and mixing it with the original modulation frequency the phase change of the reflected light is obtained, which is known as the error signal. In this case, the reflected light is used because it has the response time is faster than the transmission, because before light can be transmitted a field must build up inside the cavity, which depends on the finesse of the cavity.

In our experiment, the sidebands are created by a modulation applied in the current supplied to the laser diode. The set up used in our experiment for PDH is shown in Fig. 26. From this figure, it is possible to see that the modulated light passes first through a half-wave plate and a polarizing beam splitter which will divide the light to the ultrastable cavity and the injection lock system. In the cavity path, the laser beam shines a quarter wave plate $(\lambda / 4)$ that is used to rotate the polarization of the reflected beam by $90 \circ$ in order to maximize its recapture onto a photodiode. The signal from the photodiode 


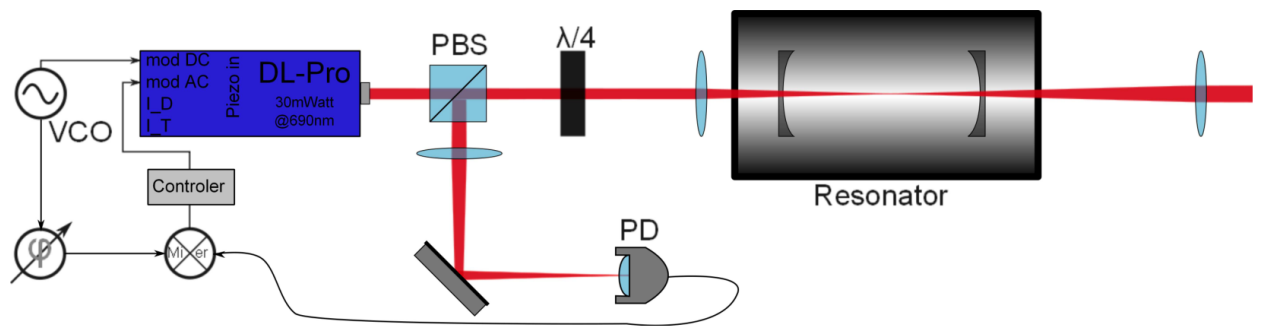

Figure 26 - The optical setup for a PDH stabilization. A VCO modulates sidebands onto the laser, which propagates to the cavity, where it is reflected. The reflected beam is separated using a $\lambda / 4$-plate and a polarizing beam splitter (PBS). The intensity is registered by a photodiode (PD).

Source: By the author.

is mixed with the modulation signal in order to make possible the detection of the phase differences, which is the error signal. The error signal is then fed into a PID controller that creates a correction signal, which is sent to the laser current controller that modifies the current to bring the laser back to the resonant frequency for the cavity.

A modulation of the laser frequency $\omega=2 \pi \nu$ by an RF frequency $\Omega$ causes sidebands that will appear in $\omega-\Omega$ and $\omega+\Omega$. The electric field of the modulated light has a modulation strength of $\beta$ will be

$$
E=E_{0}=\left[J_{0}(\beta) e^{i \omega t}+J_{1}(\beta) e^{i(\omega+\Omega) t}-J_{1}(\beta) e^{i(\omega-\Omega) t}\right] .
$$

In this case, the size of the sidebands are given by Bessel functions denoted by $J_{\alpha}$, where $\alpha$ is an integer denoting which order of Bessel function. $J_{0}(\beta)$ is the proportion of the power in the carrier frequency and $J_{1}(\beta)$ is the power in the first order sidebands. The power in the carrier is $P_{c}=J_{0}^{2} P_{0}$ and the power in the side band is $P_{c}=J_{1}^{2} P_{0}$, where $P_{0}$ is the total power of the laser beam. In this equation higher order Bessel functions have been disregarded as they are usually small because the modulation amplitude should not be chosen too strong otherwise they will produce undesired offsets.

The measurement of the phase of the reflected light is not direct. It is done by the interference processes of the carrier and sidebands in the reflected light which will create beat pattern whose amplitude is the error signal. This error signal is given by

$$
E_{r e f}=E_{0}\left[F(\omega) J_{0}(\beta) e^{i \omega t}+F(\omega+\Omega) J_{1}(\beta) e^{i(\omega+\Omega) t}-F(\omega-\Omega) J_{1}(\beta) e^{i(\omega-\Omega) t}\right],
$$

where the reflection coefficient is the ratio of the incident and reflected light $F(\omega)=$ $E_{\text {ref }} / E_{\text {inc }}$, which is dependent on the frequency of the light.

The processes of correction in the laser frequency can be understood in the following way: if the frequency of incident light and the reflected light are on resonance there will be no reflection and no phase shift of the carrier which results in a zero error signal. But when the frequency is slightly off resonance there will be a non-zero signal and the sign of the error signal will indicate in which direction a correction to the laser frequency should be done. The transmitted intensity and the error signal from the experiment when the laser frequency is scanned over a cavity resonance is shown in Fig. 27. 


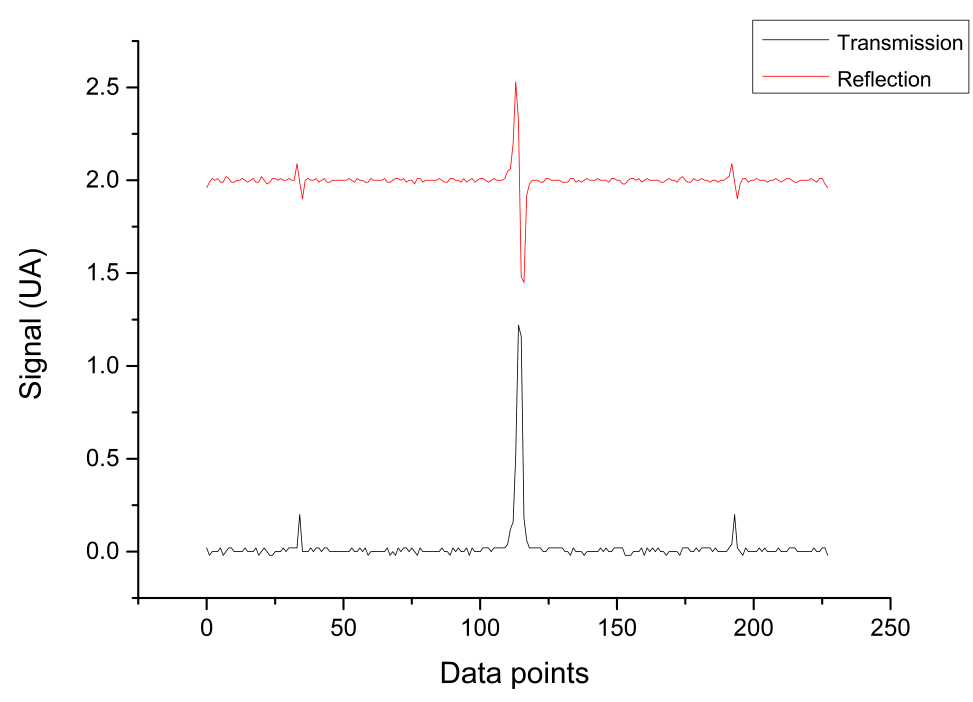

Figure 27 - Ultra-stable cavity transmitted (black) and reflected (red) signals.

Source: By the author.

\subsection{Ultra-stable High Finesse Cavities}

Trap atoms in narrow transitions, such as the red one in strontium, demands not only narrow linewidth lasers but long term stability as well. To fulfill these requirements, cavities are frequently used but they need to have well-defined frequency that will not drift with time. Named ultrastable cavities, its spacers are usually made of ultra-low expansion material and they are maintained under vacuum for temperature acoustic stabilization. As much optical frequency differentiation is required, more finesse optical cavity will increase, which implies in mirrors higher reflectances. This reflectances can reach $99.9999 \%$ can be produced, this gives a finesse of 250000 or more.

$$
\frac{\Delta \nu}{\nu}=\frac{\Delta L}{L} .
$$

To trap atoms in the ${ }^{1} S_{0^{-}}{ }^{3} P_{1}$ a finesse around few thousands is enough. This configuration provides lasers with a linewidth of few $\mathrm{kHz}$ and stability in the order of magnitude of few Hz. For clock transitions, such as the strontium ${ }^{1} S_{0^{-}}{ }^{3} P_{0}$, the required linedwith of the laser should be in the sub- $\mathrm{mHz}$ regime, which demands a cavity with at least 200000 as its finesse.

\subsection{Red laser system}

After catching and pre-cooling a sufficient amount of Sr-atoms in the blue MOT, the gas can be cooled further by making use of a narrower MOT-transition. It requires a stable and fast switch from $70 \mathrm{G} / \mathrm{cm}$ to a gradient of $5 \mathrm{G} / \mathrm{cm}$ or a storage of atoms in the quadrupole trap(28). Because the optical transition is only $7.6 \mathrm{kHz}$ wide, the laser needs to have a stability in this range. The experimental setup for the red laser is shown in Fig.28. 


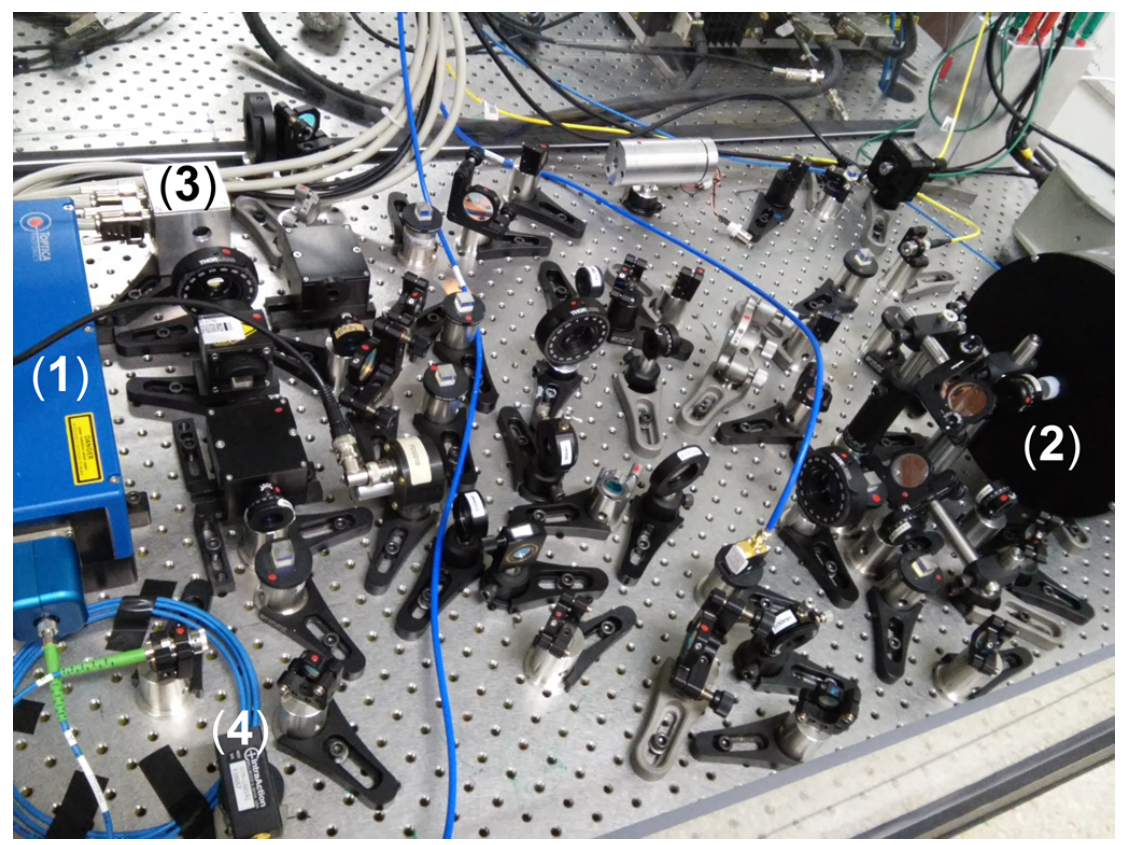

Figure 28 - Red laser system. (1) Toptica's DL pro diode laser (master laser), (2) Ultra-stable cavity, (3) Temperature and current stabilized laser diode (slave laser) and (4) 110 $\mathrm{MHz} \mathrm{AOM}$ that shifts the light frequency towards resonance.

Source: By the author.

Fig. 29 represents the red laser system. The commercial DL pro Diode laser from Toptica Photonics offers a stability of a few hundred $\mathrm{kHz}$. We improve its stability to the desired value by locking the laser electronically to an external cavity using the PoundDrever-Hall (PDH) Method. As a cavity we used a commercial Advanced Thin Films (ATF) cavity of Finesse 6000. It consists of two mirrors that are optically connected to a $10 \mathrm{~cm}$ long space tube made of ultra-low expansion (ULE) material manufactured by Corning. This material was chosen as it has a vanishing thermal expansion coefficient for a specific temperature. In our case this temperature has been measured by the manufacturer to be $35.9^{\circ} \mathrm{C}$.

The cavity is mounted within a special housing produced by Stable Laser Systems. The housing ensures that the exact temperature of the zero crossing of the thermal expansion coefficient is maintained by thermal isolation/shielding and a heating resistor, controlled with a temperature control that was build by our electronic workshop. The input for this control is an integrated type $\mathrm{F}$ thermo-resistor. Furthermore the housing provides vibrational isolation and can be evacuated in order to avoid frequency fluctuations caused by atmospheric pressure changes, which change the optical density of the air between the mirrors. In our case, the cavity is kept at a vacuum of $10^{-7}$ mbar using an ion getter pump.

After optimizing the PID-parameters of the regulation-electronics, we achieved a Allan Variance of less than the natural linewidth, which is sufficient for operating the MOT. As the resonance frequency of the cavity is no-tunable, we use AOMs in order to shift the laser that is stabilized to a cavity mode close to the atomic resonance. Using an ultra-precise wavelength-meter in combination with a Doppler free spectroscopy setup, we found a cavity mode $965 \mathrm{MHz}$ below the atomic transition. Two AOMs, one running at $840 \mathrm{MHz}$, the other at $110 \mathrm{MHz}$ shift the frequency of the light to the atomic resonance. 


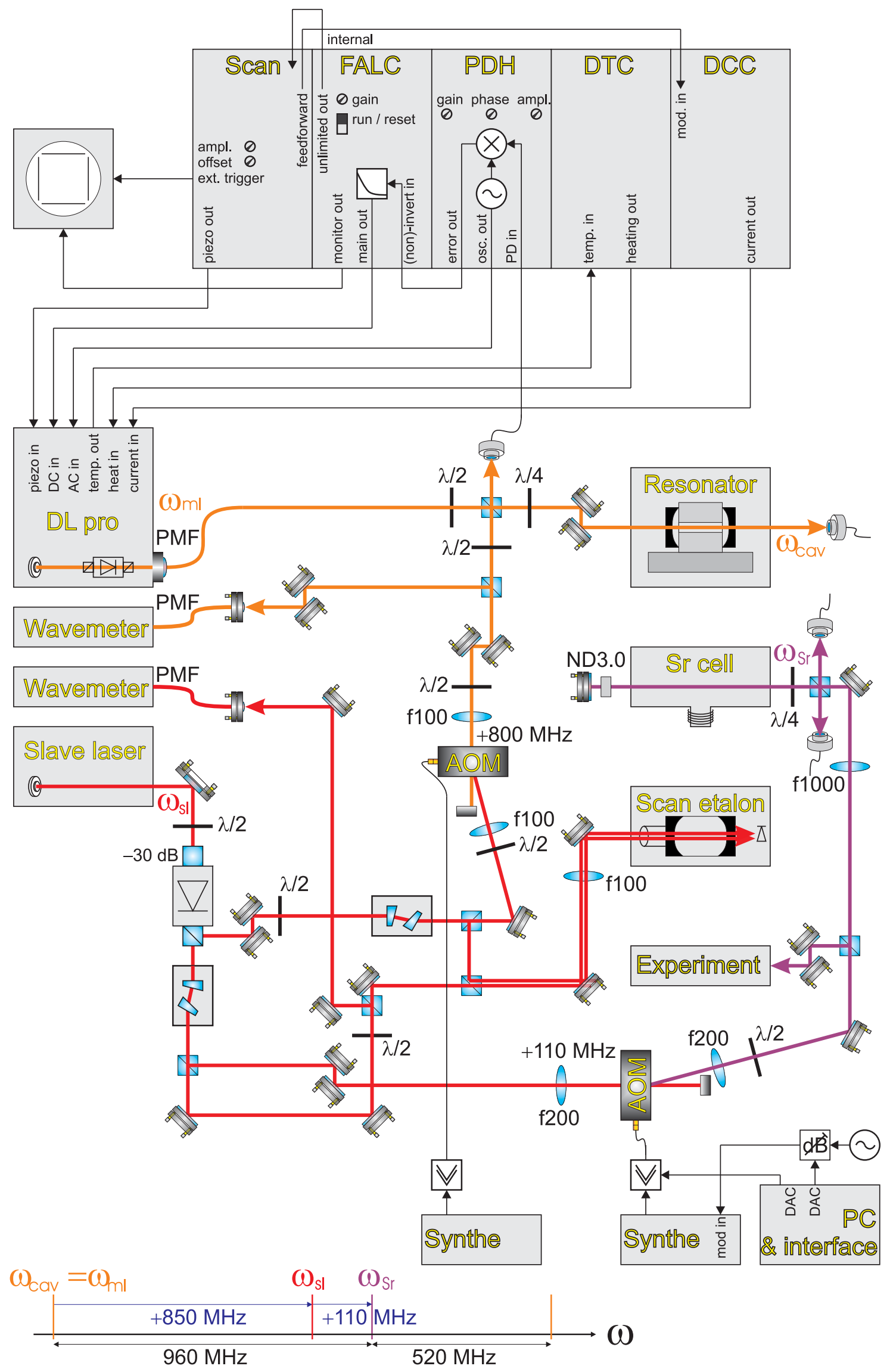

Figure 29 - Schematic representation of the red laser system.

Source: By the author. 
The output power of the diode laser is only about $8 \mathrm{~mW}$, which is used for locking it to the cavity resonance and to perform injection locking on another red laser diode. Thus, part of the light from the commercial Toptica laser, named master, is injected in a slave laser diode sitting inside a self-designed temperature-stabilized housing running at constant current. In order to eliminate unwanted optical feedback to the master, a polarizing beam splitter (PBS) cube and an optical isolator are between the last steering mirror and the slave laser. Alignment is crucial, making it necessary to optimize this alignment every few weeks. To check if the technique is working, we look at the transmission modes of the diagnostic confocal Fabry-Perot cavity. Both the slave and the master have diagnostic beams aligned to this cavity. As the length of the cavity is changed via a PZT actuator, transmission modes appear. In the absence of injection-locking, there are two sets of cavity modes: one corresponding to the master and one corresponding to the slave. If the injection-locking is working well, there will be one set of modes. Sometimes it is necessary to adjust the current of the slave laser for the injection locking to work well.

\subsection{Improved temperature stabilized laser diode housing for injec- tion lock}

As pointed in the previous section, the laser light to be used to trap atoms in the red narrow transitions is obtained by using injection-lock technique. One of the main requirements is the development of laser diode housing which provides good temperature stability and acoustic noise reduction. Ease of access to its inner parts for adjustments is also desirable. Our homemade housing (see Fig. 30) is composed two aluminum parts: the basis (Fig. Fig. 30(a)) and the collimation tube/laser diode holder (Fig. 30(b)); and an protective Plexiglas box (Fig. 30(c)). All these parts were produced and machined at IFSC's mechanical workshop. The Plexiglas box was designed to thermally isolate the
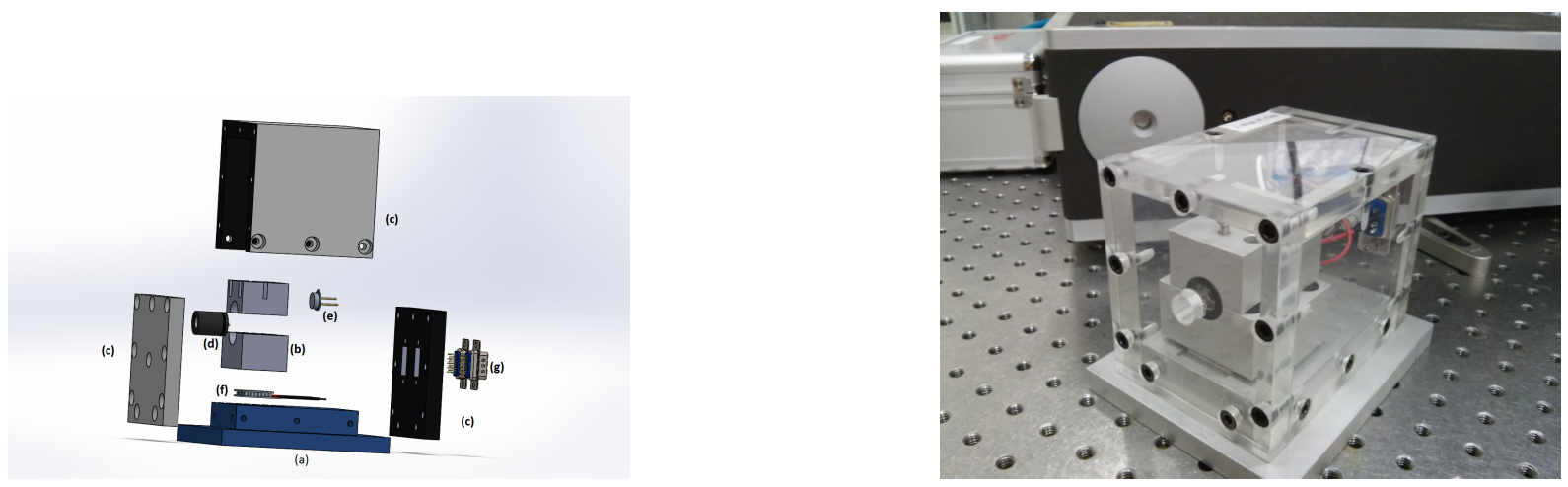

Figure 30 - Homemade laser diode housing. Left: (a) The aluminum base, (b) collimation tube holder (aluminum), (c) plexiglas protection, (d) Tholabs LTN330 collimation tube, (e) thermistor used as a temperature monitor, (f) Thorlabs TEC 3-6 and (g) D-Sub 9 connectors. Right: Picture.

Source: By the author.

lasers from the outside environment and keep dust and other unwanted particles from settling on the optics inside the boxes. By thermally insulating the lasers with protective boxes, we improve the laser temperature control even further and reduced the amount of power needed to supply it. 
An TEC element (Thorlabs TEC 3-6) is placed between the collimation tube holder and the basis to control the diode laser temperature. Both parts are held together by nylon screws to avoid thermal losses. A collimation tube (Thorlabs LTN330) holds the laser diode and with its adjustable aspheric lens collimates the output laser beam. The diode and TEC element are supplied by homemade circuit drivers (which stabilizes the current and temperature in the LD) via D-sub 9 connectors.

These homemade housings are useful and cheap. The housing price is of the housing is few hundred reais (including aluminum, plexiglass and the manufacturing process) but its overall expenses will depend on the laser diode and optics which will be used. From them, we could generate more red laser light for the second stage of our magneto-optical trap (see Chapter 4) and blue light from an low cost and high power $461 \mathrm{~nm}$ (see Chapter $5)$.

\subsection{Red MOT}

All the red laser beams have a waist of $3 \mathrm{~mm}$ at the location of the MOT and maximum power of $4.5 \mathrm{~mW}$ in each beam. It is overlapped with the blue laser beam on dichroic mirrors. In order to transfer a high percentage of atoms from the blue MOT to the red MOT, the frequency spectrum of the light has to be artificially broadened (value between 5 and $10 \mathrm{MHz}$ ), because the atomic velocities arising from the blue MOT are still too high. We do this by modulating the frequency of the AOM, generating 15 Sidebands of $30 \mathrm{kHz}$ spacing.

The transfer process is divided in three different parts: the broadband red MOT, the reduction of the artificial broadening and the single frequency red MOT. The first stage have lasts for $50 \mathrm{~ms}$ when the blue MOT atoms are transferred to the narrow transition and the gradient of magnetic field should be fast switch from $70 \mathrm{G} / \mathrm{cm}$ to 5 $\mathrm{G} / \mathrm{cm}$. An alternative method to the fast switch off of the coils is to transfer the atoms to the magnetic trap. In this stage, the saturation parameter in the MOT beams is equal to few thousands to improve this recapture process. In the next $200 \mathrm{~ms}$ the broadening is ramped down (from $5 \mathrm{MHz}$ to $1 \mathrm{MHz}$ ) and the intensity reduced (the $s$ parameter used is in the order of magnitude of hundreds) to cool down futhermore the atoms. Finally, the red MOT reaches a single frequency regime by removing the modulation and bring the detuning of the red light to few linewidths. This last stage lasts $50 \mathrm{~ms}$ followed by $50 \mathrm{~ms}$ in the final values to stabilize the trap.

With the procedure described above we were able produced once $10^{6}$ atoms at an estimated temperature of $20 \mu \mathrm{K}$, once Doppler and recoil temperature are $179 \mathrm{nK}$ and $460 \mathrm{nK}$, respectively. The number of atoms and the temperature can be improved by playing with the laser beam parameters(29-31). Unfortunately, the capture of the precooled atoms in the narrow red transition revealed to be dramatically unstable, in the Section 4.9 I will present all the characterizations done in order to approach a stable reproducibility. 


\subsection{Red MOT attempts}

In this section, I will present all the measurements we did to characterize our red laser system and to find what is the reason to do not produce stable and reproducible ultracold samples of strontium. The measurements performed include check the stability and the mode spectrum of the injection locked light, check the free spectral range the ultra stable cavity and an attempt to override the slow switch off time of the quadrupole coils.

In which concern about the red laser system stability, an heterodyne beat measurement were performed to check if the slave laser light is has the same spectrum is equal to the master laser and to check if the modulation is transferred in the injection lock process (see Fig. 31). From these measurements, it is possible to conclude that all the slave light is composed only by master laser light and that the modulation is also completely transferred.

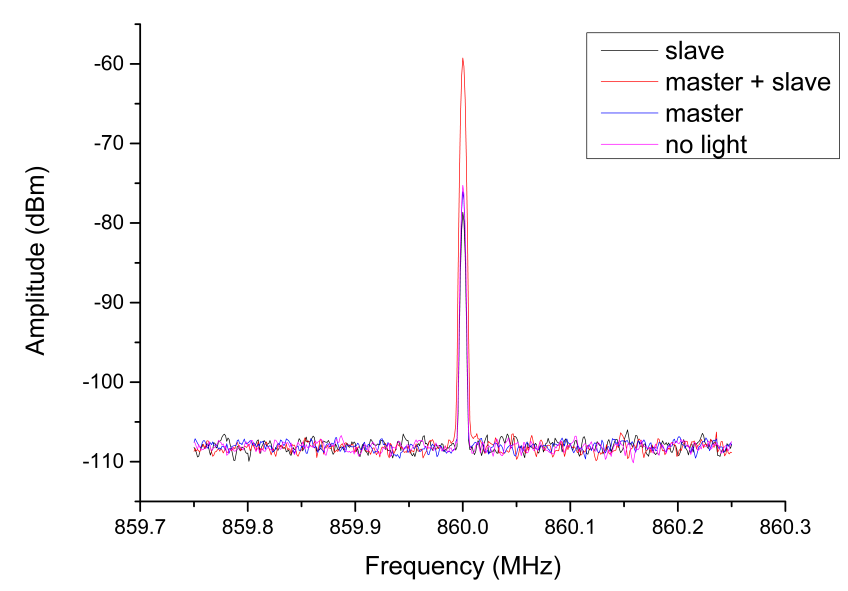

Figure 31 - Heterodyne beat signal between red master and slave laser.

Source: By the author.

\subsubsection{Ultra-stable cavity free spectral range measurement}

In order to check the stability of our ultra-stable cavity, which is used as a reference for all the red laser system, a measurement of its free spectral range was performed. To this purpose, a optical frequency comb, FC1500-250-WG from Menlo systems, equipped with 689 and $698 \mathrm{~nm}$ modules (for more details about frequency combs and its application, see (32)), was used. All the steps are described below.

The red laser was locked at two different modes of the reference cavity, whose frequencies measured with the High Finesse WS6 wavelength meter were found to be close to

$$
\nu_{w m, a}=434.7848 \mathrm{THz}
$$

and

$$
\nu_{w m, a}=434.7788 \mathrm{THz} .
$$


The two modes were 4 free spectral ranges (FSR) apart from each other. From this we derive a first estimation of the fsr, $\delta_{f s r}=\nu_{w m, 2}-\nu_{w m, 1}=1.5 \mathrm{GHz}$.

Simultaneously, comparison of the laser frequency with the frequency comb yielded $\nu_{f c, a}=434.784786973 \mathrm{THz}$ and $\nu_{f c, a}=434.778803764 \mathrm{THz}$ giving a better estimation of the FSR, $\delta_{f s r}=\nu_{w m, 2}-\nu_{w m, 1}=1.495802 \mathrm{GHz}$.

Knowing that $\nu_{f c} / \delta_{f s r}$ must give an integer number, we obtain

$$
N_{a}=\frac{\nu_{f c, a}}{\delta_{f s r}}=290670
$$

and

$$
N_{b}=\frac{\nu_{f c, b}}{\delta_{f s r}}=290666
$$

which, in turn, allows us to fix the fsr with higher precision

$$
\delta_{F S R}=\frac{\nu_{f c, a}}{\delta_{f s r}}=1.49580206754 G H z=\frac{\nu_{f c, b}}{\delta_{f s r}},
$$

Finally, we locked the laser to a third mode closer to the strontium line. With $N_{c}=290699$ we expect the cavity resonance at $\nu_{C a v}=434.82816523 \mathrm{THz}$.

The strontium line for the ${ }^{1} S_{0^{-}}{ }^{3} P_{1}$ transition is equal to $\nu_{S r}=434.829121311$ THz. We observe this line shining the red laser into a blue MOT increasing the master laser frequency resonant to its cavity by $+840 \mathrm{MHz}$ and $+150 \mathrm{MHz}$. Hence, the cavity resonance is around $\nu_{\text {Cav }}=434.828134 \mathrm{THz}$. It did not change more than a few $\mathrm{MHz}$ in years!

Recalibrating the FSR using this information:

$$
\delta_{F S R}=\frac{\nu_{S r}}{N_{C}}=1.495801960(3) G H z,
$$

; assuming a $1 \mathrm{MHz}$ uncertainty in hitting the resonance with the red laser coming from the Doppler width of the blue MOT.

\subsubsection{Magnetic trap lifetime measurements}

Instead of a fast switch of the magnetic fields in the experiment, atoms in blue MOT can be accumulated in a magnetic trap, using a continuous loading scheme based on optical pumping into a metastable state. Then, they are pumped back into electronic ground state and laser cooled using the red narrow intercombination line.

The magnetic trap is analyzed by performing a MOT recapture after a holding time. For comparison the MOT timescales are also shown in Fig. 32. The steady state atom number is determined by the balance between loading and loss rates. The magnetic trap should only be limited by vacuum, while the MOT has additional losses due to the incident light. However, magnetic traps are extremely sensitive to any background resonant light. So, in order to achieve the vacuum-limited lifetime one should block all light sources from the experiment during magnetic trapping

All measurements are taken using the MOT fluorescence focused onto a photodiode; the green is controlled with a mechanical shutter and measured on a photodiode. 


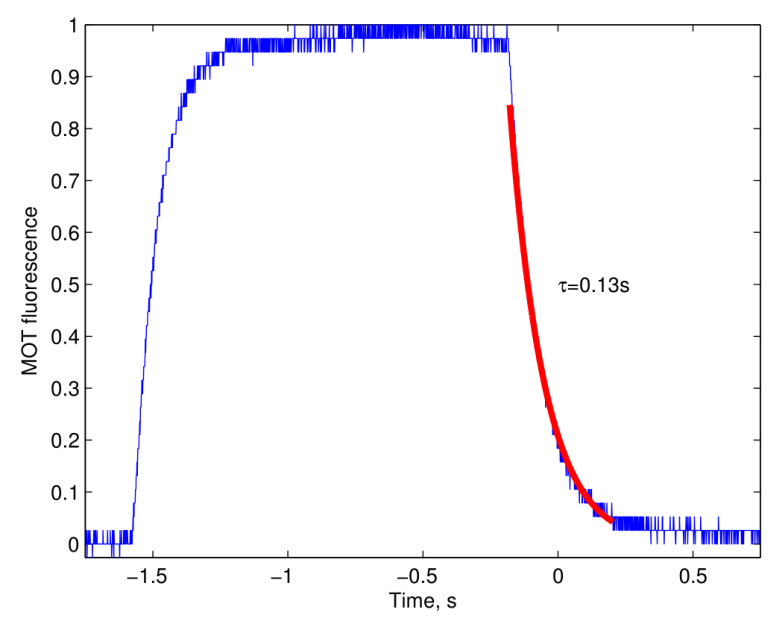

Figure 32 - MOT loading and decay times. Initially all beams are on, then the Zeeman slowing beam is blocked and an exponential decay begins. Fit gives a decay time of $0.13 \mathrm{~s}$.

Source: By the author.

The recapture peak is normalized to the MOT fluorescence in order to account for intensity/frequency variations. From the results we can see that the magnetic trap lifetime is certainly sufficient. Also, the magnetic trap takes a significantly longer time to load than the MOT. Thus it either contains a higher steady state atom number, or has a slower loading rate. As the number of atoms in the magnetic trap is most likely equal or greater than the number of atoms in the blue MOT (due to lower loss rates), we may be able to expect that the recapture peak could be brought above the initial MOT fluorescence. Fig.33(a) shows the highest performance that we could achieve, showing that $75 \%$ of the atoms can be transferred. Fig.33(b) shows that the lifetime of the magnetic trap is $2.4 \mathrm{~s}$, which we assume to be limited by the background vapor pressure.

In addition to these results, the background (i.e., any spurious light originated in the laboratory or the laser systems) after equal time was measured. No background fluorescence could be measured. It was also checked that a flash of green in the hold time destroyed the magnetic trap.

\subsubsection{Conclusions}

Unfortunately, we got no operating red MOT. Further investigations will be performed and soon we hope to solve all the problems and then create ultra-cold samples of strontium. 

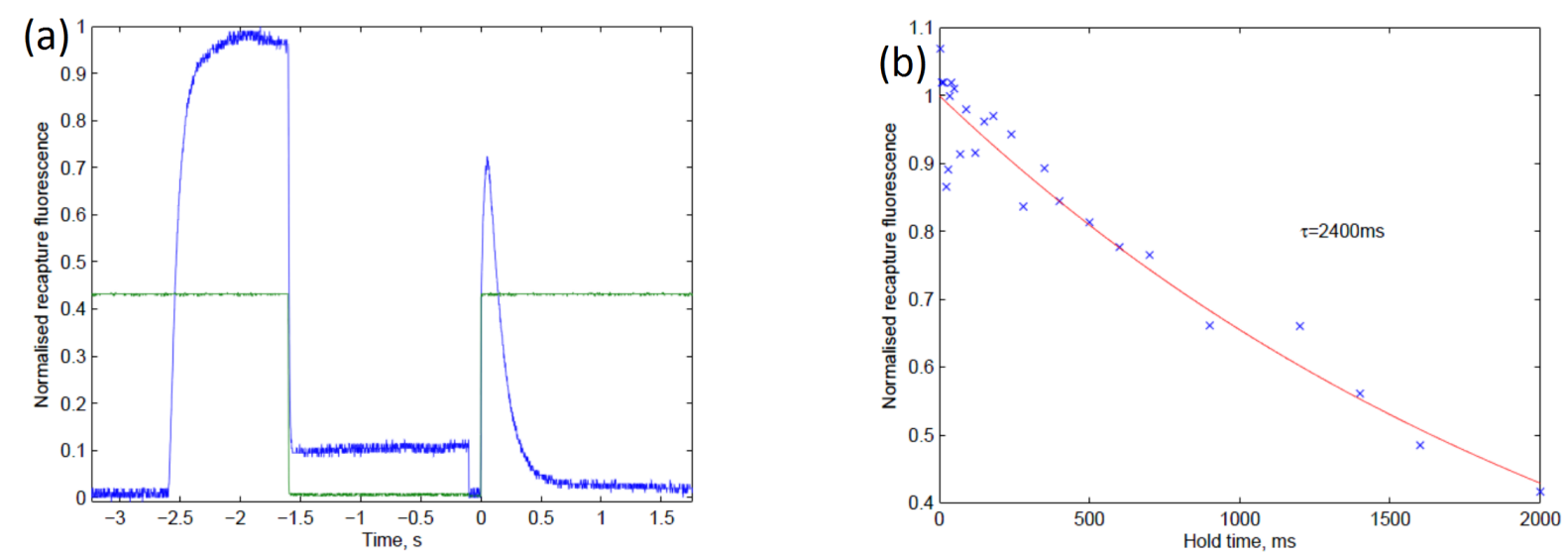

Figure 33 - Transfer of atoms into the magnetic trap for a MOT number of $2 \times 10^{8}$ atoms. The atoms are first cooled in a MOT, and then the repumper blocked. This pumps cold atoms into the magnetically trapable dark state. The light is then switched off for a hold time of at least $100 \mathrm{~ms}$ to allow untrapped atoms to vacate the MOT region. A recapture measurement is then performed by pulsing on the repumper and counting the number of atoms that radiate in the MOT. The lifetime of the magnetic trap were found by varying the hold time. (a) Recapture efficiency; and (b) Lifetime measurements.

Source: By the author. 


\title{
5 Injection locking of a low cost high power laser diode at $461 \mathrm{~nm}$
}

\author{
Authors: C.H.J. Pagget*, P.H. Moriya*, R.C. Teixeira*, R.F. Shiozaki*, \\ M. Hemmerling* ${ }^{*}$ Ph.W. Courteille*
}

\author{
Same text of the paper submitted to arXiv: \\ http://arxiv.org/abs/1601.03553
}

Stable laser sources at $461 \mathrm{~nm}$ are important for optical cooling of strontium atoms. In most existing experiments this wavelength is obtained by frequency doubling infrared lasers, since blue laser diodes either have low power or large emission bandwidths. Here, we show that injecting less than $10 \mathrm{~mW}$ of monomode laser radiation into a blue multimode $500 \mathrm{~mW}$ high power laser diode is capable of slaving at least $50 \%$ of the power to the desired frequency. We verify the emission bandwidth reduction by saturation spectroscopy on a strontium gas cell and by direct beating of the slave with the master laser. We also demonstrate that the laser can efficiently be used within the Zeeman slower for optical cooling of a strontium atomic beam.

\subsection{Introduction}

Motivated by the existence of narrow and ultranarrow transitions in strontium atoms the demonstration of optical cooling and trapping $(8,33)$ and recently the BoseEinstein condensation $(29,34)$ of strontium gases propelled this species among the hottest candidates for optical frequency standards $(35,36)$. The strongest atomic resonance, $\left(5 s^{2}\right)^{1} S_{0}-(5 s 5 p)^{1} P_{1}$, which has a transition wavelength of $460.86237 \mathrm{~nm}$ and a linewidth of $30.5 \mathrm{MHz}$, serves as a cooling transition in magneto-optical traps down to a few $\mathrm{mK}$. As the saturation intensity of this transition is quite large, $I_{\text {sat }}=40 \mathrm{~mW} / \mathrm{cm}^{2}$, relatively large laser power on the order of several $100 \mathrm{~mW}$ is required for efficient cooling in magneto-optical trap arrangements. Most laboratories working with strontium obtain the necessary power by frequency doubling an infrared laser operated at $922 \mathrm{~nm}$. Although efficient, this option is rather cumbersome and expensive.

High gain and stable operation frequently are antagonistic requirements in laser diodes, especially in the wavelength range near $461 \mathrm{~nm}$, where the panoply of blue laser diodes available on the market is sparse. A possible remedy is the use of the injection locking technique. Here, a high-power laser, called the slave laser, sees its noise level strongly reduced by injection of light from a low-noise low-power master laser into the slave laser's active zone. Provided that the frequencies of the master laser and the free-running slave laser are sufficiently close, the injection forces the slave laser to operate exactly on the injected frequency with little noise. The higher the injected power, the larger is the

\footnotetext{
*Instituto de Física de São Carlos, Universidade de São Paulo, São Carlos, Brasil
} 
allowable frequency offset between the seed laser and the slave laser's resonance (37). Close to quantum-limited intensity and phase noise can be achieved with this technique $(38-42)^{\dagger}$.

Recently, Y. Shimada et al. used expensive $100 \mathrm{~mW}$ single-mode laser diodes from Nichia (Nichia, NDB4216E-E), specified for a wavelength between $450 \mathrm{~nm}$ and $460 \mathrm{~nm}$, to perform spectroscopy on a strontium hollow cathode (43). Two identical laser diodes were used in this experiment: A first laser diode was forced to operate near the Sr transition wavelength by optical feedback within an extended cavity setup (ECDL). When more than $20 \mathrm{~mW}$ of its power were injected into a second, free-running laser diode of the same type, the authors reached up to $110 \mathrm{~mW}$ of useful single-mode laser emission from the injected diode.

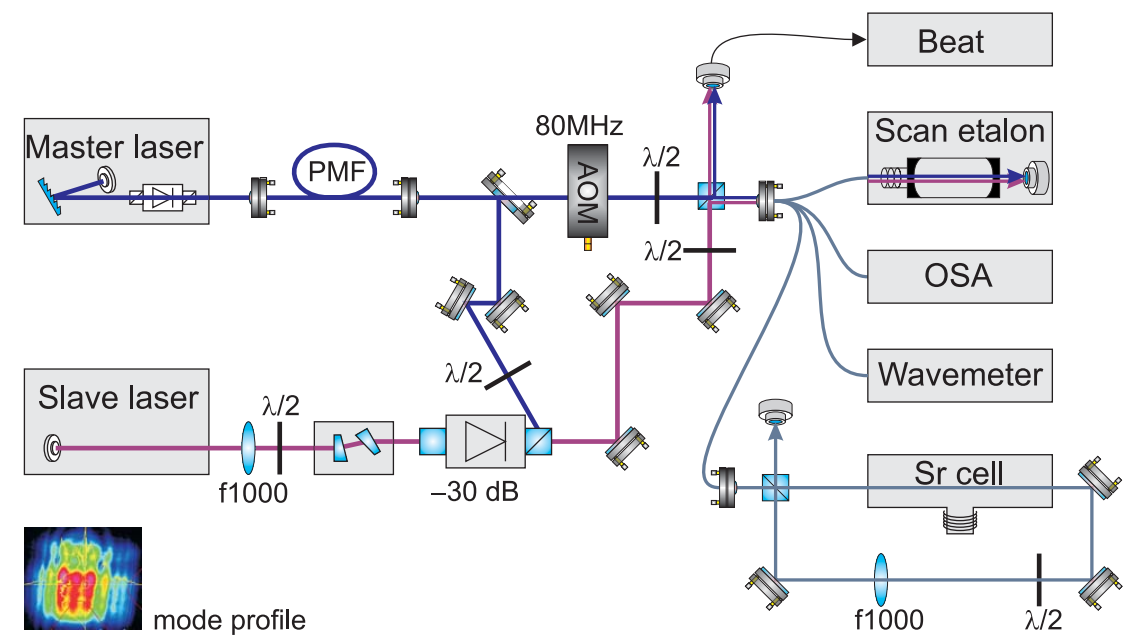

Figure 34 - (color online) Optical setup for injection locking. A single mode tunable master laser injects, through an optical isolator, the slave laser diode. The emission spectra of both lasers are simultaneously monitored with a wavemeter, an optical spectrum analyzer (OSA), and a scanning etalon. Also shown is the setup for beat frequency measurement and for saturation spectroscopy on the strong cooling transition of strontium at $461 \mathrm{~nm}$. (ND3: 3dB neutral density filter, PMF: polarization maintaining fiber: AOM: acousto-optic modulator) Also shown is a typical spatial mode profile of the slave laser observed with $200 \mathrm{~mA}$ laser current.

Source: By the author.

Cheap high power multi transverse mode laser diodes near $462 \mathrm{~nm}$ are nowadays sold for laser pointer and SciFi games applications ${ }^{\ddagger}$. Here, we report on an injection locking experiment, where we harness the high power (nominally $1.4 \mathrm{~W}$ ) multimode laser diode NDB7675 from Nichia with up to $\sim 10 \mathrm{~mW}$ of single-mode laser light. We also tested a M462 laser diode with $2 \mathrm{~W}$ nominal power ${ }^{\S}$. The slave laser emits up to $430 \mathrm{~mW}$ of light power up to $50 \%$ of which are slaved to the injected frequency. The limitation in the slave laser power comes from our stable current supply, which can afford a maximum current of $500 \mathrm{~mA}$. The front window of the slave laser diode case is removed, in order to avoid

\footnotetext{
${ }^{\dagger}$ Technical note: http://assets.newport.com/webDocuments-EN/images/App-Note-InjectionLocking.pdf.

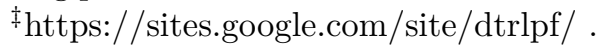

$\S$ According to https://sites.google.com/site/dtrlpf/ these laser diodes are extracted from Casio XJM140 projectors.
} 
competition between the injection locking and the optical feedback on the slave diode from the reflection on the front window. The main limitation arises from the multiple transverse modes emitted by the slave laser (see Fig. 34), which cannot perfectly be matched with the Gaussian mode profile of the master laser. Nevertheless, we find that the injected light efficiently narrows the slave laser emission bandwidth and even reduces the transverse mode spectrum.

We demonstrate the viability of the proposed scheme by a master-slave frequency beating measurement, by saturated absorption spectroscopy on a hot vapor strontium cell, and by laser-cooling of an atomic beam in a Zeeman slower. The master-slave locking scheme thus represents an efficient cheap and compact solution for a laser source driving the main strontium line and might reveal itself an asset in the future realization of transportable optical strontium clocks.

\subsection{Experimental set-up}

The experimental layout of the injection locking is shown in Fig. 34. The master laser, a Toptica TA-SHG pro, consists of an ECDL laser at $922 \mathrm{~nm}$, amplified by a tapered amplifier and frequency-doubled to $461 \mathrm{~nm}$. Part of its power is coupled to an optical fiber (PMF) delivering up to $10 \mathrm{~mW}$ power with a frequency bandwidth below $1 \mathrm{MHz}$. The main fraction of this light is injected through an optical isolator (Thorlabs, IO-3-460-HP) into the slave laser. The remaining power is frequency-shifted by $130 \mathrm{MHz}$ with an acoustooptic modulator (AOM) and superposed with the light from the slave laser. The slave laser is the Nichia laser diode NDB7675. Its temperature is actively controlled with $10 \mathrm{mK}$ precision. Both, the master and the slave laser frequencies are simultaneously monitored with a wavemeter (HighFinesse, WS6), an optical spectrum analyzer (HighFinesse, LSA), and a home-built scanning Fabry-Pérot etalon with a finesse of 50, as illustrated in Fig. 34 .
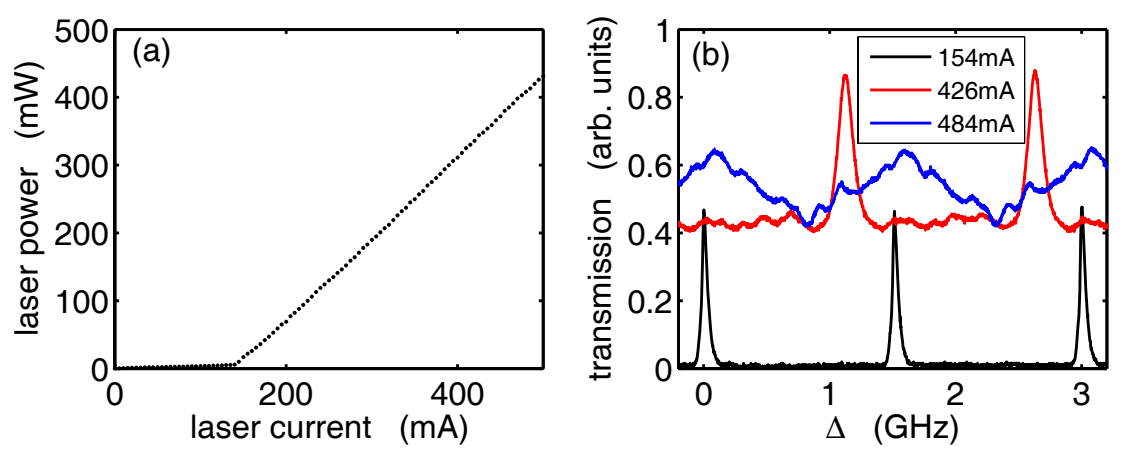

Figure 35 - (color online) (a) Measured total emission power of the free-running laser diode as a function of laser current. The lasing threshold is at a current of $140 \mathrm{~mA}$, the slope efficiency is $1.2 \mathrm{~W} / \mathrm{A}$. (b) Emission spectrum of the free-running laser diode measured with a scanning Fabry-Pérot interferometer at low (black) and high (red and blue) laser currents.

Source: By the author.

The Nichia laser diode has a nominal power of $1.4 \mathrm{~W}$ with a several nanometer large spectrum centered at $462 \mathrm{~nm}$ and multiple transverse modes. Fig. 35(a) shows the measured laser power emitted by the free-running laser diode as a function of current. 
Fig. 35(b) shows the emission spectrum recorded with a home-built scanning Fabry-Pérot interferometer with $1.5 \mathrm{GHz}$ free spectral range and a finesse of 50. While at low $(154 \mathrm{~mA})$ current the spectrum is single mode, at higher current the spectrum becomes very broad, which is the cause for the large offset appearing in the spectrum of Fig. 35(b) for $426 \mathrm{~mA}$ current and the complete loss of the mode structure for $484 \mathrm{~mA}$ current.
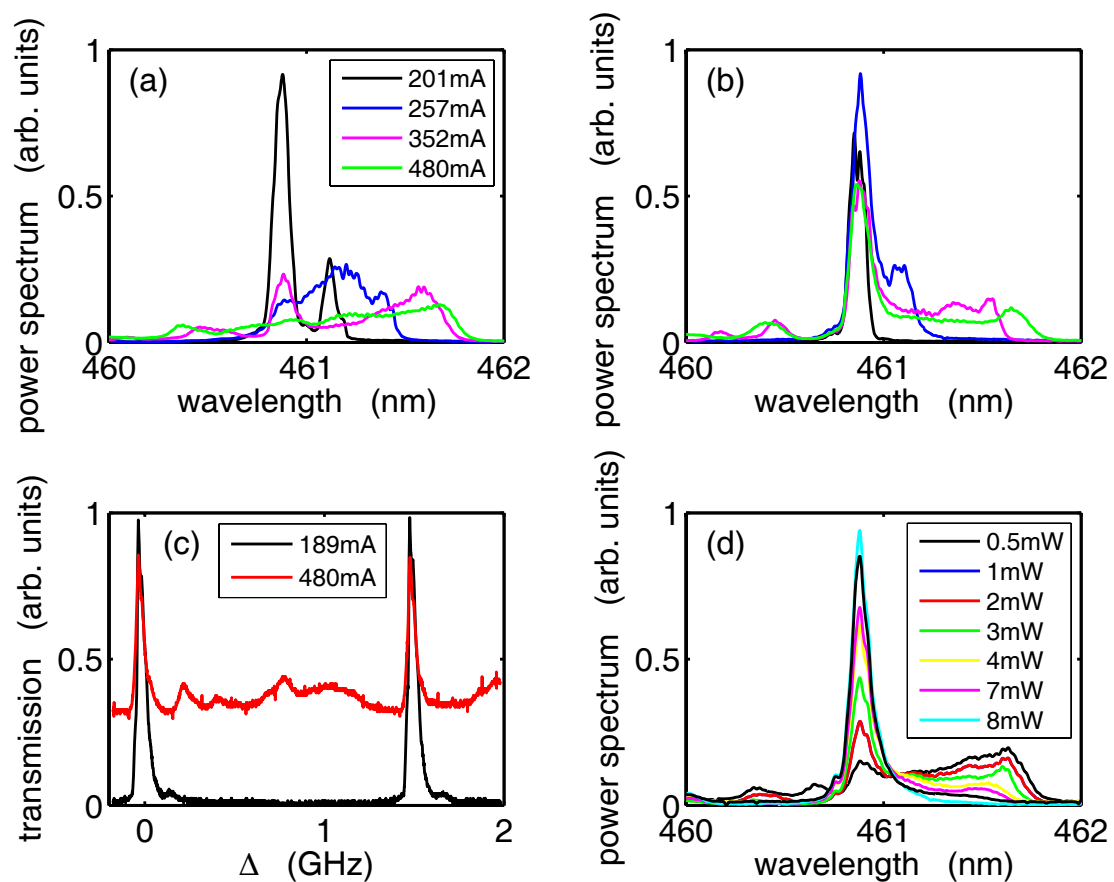

Figure 36 - (color online) (a) Emission spectrum of the free-running laser diode measured with an optical spectrum analyzer at various laser currents. The temperature of the laser diode was optimized for each current in order to maximize the power in the master frequency. (b) Same as (a) but with $0.5 \mathrm{~mW}$ injected light power from the master laser. (c) Emission spectrum of the slave laser injected with $0.5 \mathrm{~mW}$ measured with a scanning Fabry-Pérot interferometer at low (black) and high (red) laser current. (d) Same as (a) but for a fixed laser current of $477 \mathrm{~mA}$ and varying the injection power.

Source: By the author.

\subsection{Characterization of injection locking}

In order to narrow the emission spectrum, we inject into the laser diode between 0.5 and $10 \mathrm{~mW}$ of light from the master laser. The spectra are recorded with an optical spectrum analyzer (High Finesse, LSA) with $6 \mathrm{GHz}$ resolution and shown in Figs. 36(ac). The spectra shown in Fig. 36(a) are obtained for various slave laser currents without injection. While at low current the power concentrates around a relatively narrow spectral region, at higher currents the spectra become much broader and almost continuous. Figs. 36 (b) are recorded with injection of $0.5 \mathrm{~mW}$ single mode radiation at $460.86 \mathrm{~nm}$. The Doppler-free Lamb-dip is clearly visible is the enhanced purity due to the injection: At low currents the power spectrum appears to become single-mode near the master laser frequency. At higher currents, however, there is still a pedestal in the spectrum. This 
behavior of the slave laser is confirmed via the transmission spectrum of the scanning Fabry-Pérot etalon: Fig. 36(c) shows how, despite $0.5 \mathrm{~mW}$ injected power, the slave laser broadens its spectrum when its current is increased from $189 \mathrm{~mA}$ to $480 \mathrm{~mW}$. The spectrum can further be purified by increasing the power of the injected light. Fig. 36(d) shows the narrowing of the spectra as the injection power is increased. At $10 \mathrm{~mW}$ of injection power no frequency components are discernible beyond the master laser frequency within the resolution of the optical spectrum analyzer.

\subsection{Measurements}

We demonstrate the performance of the injection locking system via three different measurements, 1. a master-slave frequency beat, 2. spectroscopy on a Sr cell, and 3. application in laser-cooling.

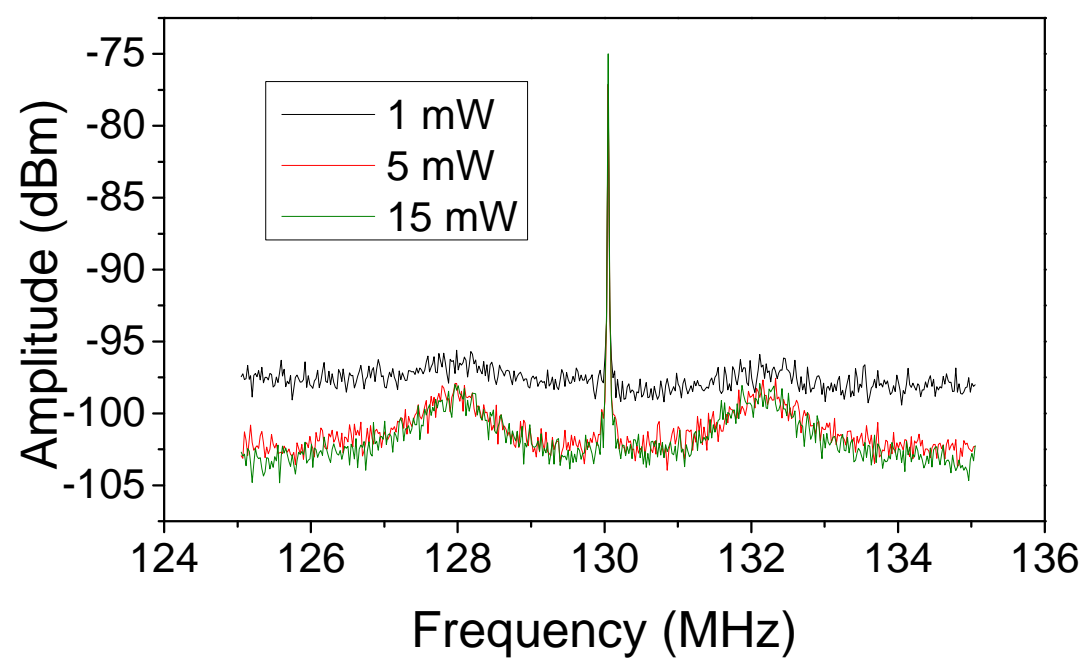

Figure 37 - (color online) Beat frequency spectrum between the master laser frequency-shifted by an $\mathrm{AOM}$ at $130 \mathrm{MHz}$ and the injected slave laser. The line is stable and its resolution only limited by the resolution bandwidth (here $100 \mathrm{~Hz}$ ) of the spectrum analyzer and the frequency-noise of the AOM. The noise level is reduced with higher injection power at the exception of two small $1 \mathrm{MHz}$ broad sidebands at $\pm 2 \mathrm{MHz}$ to the left and right of the beat frequency.

Source: By the author.

1. We record the beat frequency spectrum between the slave laser and a reference beam obtained by frequency shifting the master laser by $130 \mathrm{MHz}$, as shown in Fig. 34 . Within the $150 \mathrm{MHz}$ bandwidth of the photodetector (Thorlabs, PDA10A) the spectrum (shown in Fig. 37) exhibits a single narrow line, whose width is only limited by the resolution of the spectrum analyzer. Interestingly, the noise floor drops with more efficient injection locking, leaving only two $1 \mathrm{MHz}$ wide sidebands at $2 \mathrm{MHz}$ distance to the left and right of the beat frequency. This shows that the injection of spectrally pure light is able to deplete the frequency components of the slave laser within a bandwidth of at least $150 \mathrm{MHz}$. The spectral purity of the beat spectrum in a large frequency band is 
encouraging for applications of the slave laser in high-resolution laser spectroscopy and narrow atomic transitions and optical cooling of atomic gases.

2. These findings do, however, not exclude the possible presence of frequency components beyond the $150 \mathrm{MHz}$ bandwidth. Such components should, in principle, appear in the spectra taken with the Fabry-Pérot interferometer [see Fig. 2(b)] or the optical spectrum analyzer [see Fig. 3(a-c)]. However, the sensitivity of the interferometer is too low and the resolution of the optical spectrum analyzer too broad.

In order to obtain information on the slave laser emission spectrum beyond the $150 \mathrm{MHz}$ bandwidth, we performed saturation spectroscopy on a $15 \mathrm{~cm}$ long strontium gas cell heated to $530^{\circ} \mathrm{C}$. The temperature, and hence the strontium partial pressure, are adjusted such as to yield a measured optical density on the order of 1 . The cell also contained an argon buffer gas at pressure 1 mbar $\$$. We attribute that to the presence of impurities in our buffer gas, which contributed to the creation of $\mathrm{Sr}$ oxide, thus changing the vapour pressure equilibrium of the cell. As shown in Fig. 34, the saturated absorption signal is obtained by passing through the $\mathrm{Sr}$ cell a saturation beam ( $8 \mathrm{~mW}$ corresponding to $20 \%$ of the saturation intensity) and a counterpropagating probe beam obtained by retro-reflecting an attenuated $(6 \mathrm{~dB})$ fraction of the saturation beam.
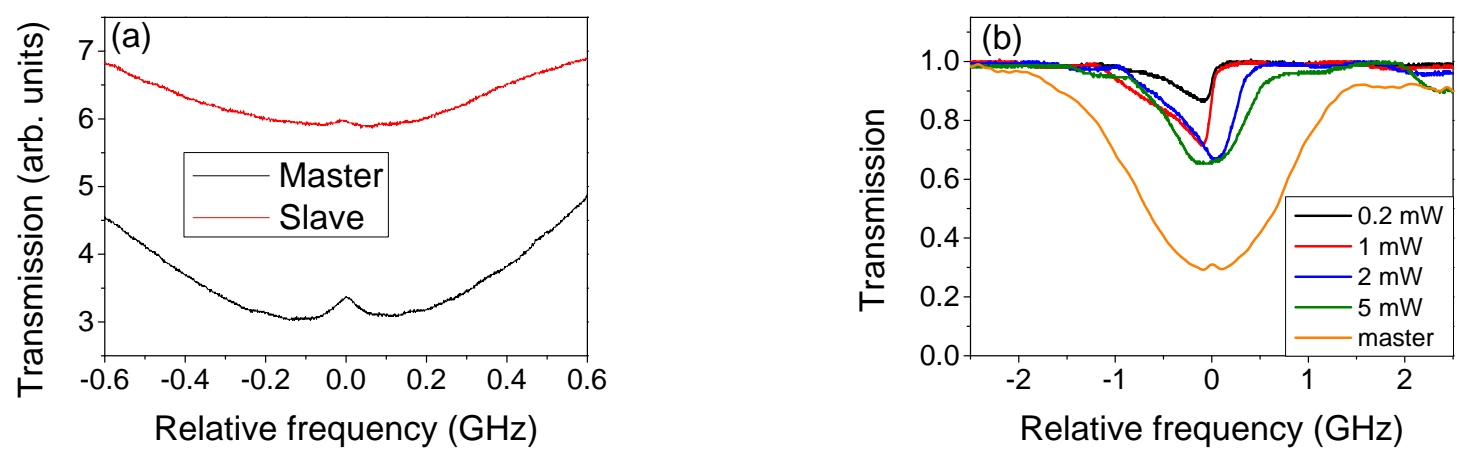

Figure 38 - (color online) (a) Saturated absorption spectra recorded with the master laser (lower black curve) and with the injection-locked slave laser (upper red curve). The injection power was $8.6 \mathrm{~mW}$ and the slave laser current $480 \mathrm{~mA}$. Both laser beams had 8 $\mathrm{mW}$ power (20\% of the saturation intensity) at the entrance of the cell. (b) Singlepass absorption spectrum of the master laser (orange) and the slave laser (other lines) for various injection powers, $1-I_{\text {trans }} / I_{\text {in }}$ versus the master laser detuning $\Delta$.

Source: By the author.

The ${ }^{1} S_{0}-{ }^{1} P_{1}$ transition of ${ }^{88} \mathrm{Sr}$ has a natural linewidth of $30.5 \mathrm{MHz}$. The master laser was ramped across the $2 \mathrm{GHz}$ large Doppler profile of this transition. Fig. 38(a) shows two spectra: The black (lower) curve is taken with the master laser itself, while the red (upper) curve is taken with the slave laser. For all spectra recorded with the slave laser, we always scan the master laser's frequency. Due to injection locking, the slave frequency follows the master. The injection locking is optimized at the center of the scan. Clearly visible is in both cases the Doppler-free Lamb-dip (FWHM 30.5 MHz). However,

\footnotetext{
I We note that the temperature of the Sr cell was higher than what should be expected from Sr partial pressure curves.
} 
the spectrum taken with the slave laser exhibits a large offset caused by a constant large background of transmitted light.

An explanation could be the existence of frequency components in the slave laser spectrum, which are not resonant with the Sr line and, hence, not absorbed. To study this further, we show in Fig. 38(b) absorption spectra of the slave laser recorded at various injection laser powers. As seen in Fig. 36(c), at low injection laser power the slave laser emission spectrum is so broad that most of the laser power is transmitted. As the injection power is increased, the emission concentrates more and more near the $\mathrm{Sr}$ resonance frequency, so that the more power is absorbed. Moreover, the spectral narrowing of the absorption spectrum points towards an efficient narrowing of the slave laser emission spectrum. However, the fact that not more than $50 \%$ of the power is absorbed shows the persistence of non-resonant frequency components beyond the range of the Dopplerbroadened resonance, which are not absorbed by the strontium gas and create the offset observed in Fig. 38(a). Note that the widths of the transmission profiles of Fig. 38(b) created by the slave laser are smaller than the width of the transmission profile for the master laser. This gives an estimation of the robustness of the injection locking when we scan the master frequency. Indeed, at about $1 \mathrm{GHz}$ from the optimized injection locking frequency, no more light is absorbed from the slave laser, while the master still shows some absorption, indicating a very poor locking of the frequency of the slave.

3. Up to this point we demonstrated spectral purity with a $150 \mathrm{MHz}$ bandwidth and the existence of frequency components beyond $2 \mathrm{GHz}$. When used for spectroscopy at atomic resonances, such as Sr, these frequency components are sufficiently far away not to disturb the atoms (the atoms are basically transparent), so that it is likely that the setup can be used, e.g. for laser-cooling purposes. Our lab disposes of an experimental setup for the preparation of cold atomic ${ }^{88} \mathrm{Sr}$ in a magneto-optical trap (MOT) loaded from a Zeeman slower: A chunk of strontium is heated to $580^{\circ} \mathrm{C}$ in an oven connected, via a two-dimensional array of microtubes, with a $35 \mathrm{~cm}$ long vacuum tube hosting a Zeeman slower in spin-flip configuration (44), where it generates a collimated atomic beam. The Zeeman slower is operated with laser light derived from the master laser tuned $530 \mathrm{MHz}$ below the atomic resonance frequency and injected in counterpropagating direction to the atomic beam. The Zeeman slower tube ends on an ultrahigh vacuum chamber, where the atoms are captured by the MOT and cooled to about $5 \mathrm{mK}$ temperature. The fluorescence recorded from the MOT is a measure for the number of trapped atoms and, hence, of the efficiency of the Zeeman slower.

In order to demonstrate that the injected slave laser light can be used for lasercooling purposes, instead of sending the Zeeman slower light beam to the main experimental setup, we use it as the master laser for the injection locking scheme, and then send the slave laser light to the Zeeman slower. Fig. 39(a) shows the resonance fluorescence of the magneto-optical trap when we turn on the light operating the Zeeman slower for both cases, i.e., the light is taken directly from the master (black curve) or indirectly from the injected slave laser. The MOT is operated by light directly derived from the master laser. The efficiency of the Zeeman slower is smaller for the slave as compared to the master for the same light intensity, because part of the slave power is in far away frequencies that do not contribute to the deceleration effect. However, as seen in Fig. 39(b), it is always possible to crank up the slave laser power to make up for the lower efficiency. 

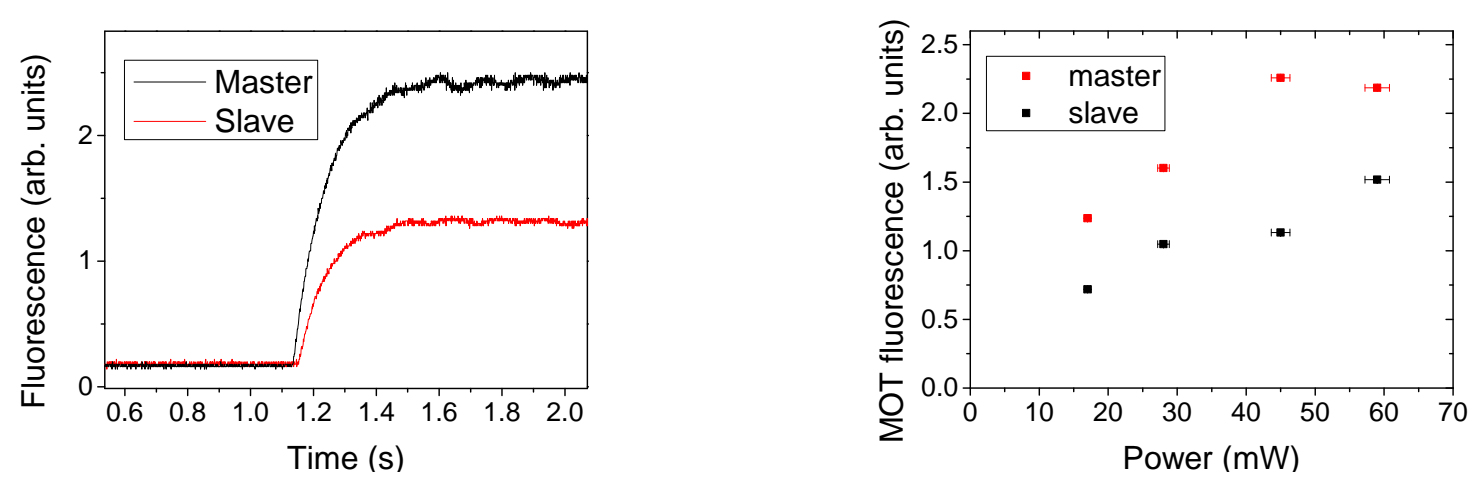

Figure 39 - (color online) (a) MOT fluorescence recorded while switching the light operating the Zeeman slower: At the time $1.1 \mathrm{~s}$ the master (black) or the slave (red) laser is suddenly irradiated into the Zeeman slower, thus loading the MOT with Sr atoms. In both cases, the master and the slave laser had the same power of $45 \mathrm{~mW}$. (b) Fluorescence measured after the MOT has been fully loaded for various lasers powers irradiated into the Zeeman slower using the master (black) or the slave (red) laser.

Source: By the author.

\subsection{Conclusion}

In conclusion, we found that injection-locked multi-transverse mode laser diodes can profitably be used in high-resolution spectroscopy. Operated at high laser powers up to $500 \mathrm{~mW}$ their transverse mode profile becomes increasingly broad, which hampers a perfect mode-matching of the injection beam. Nevertheless, at sufficiently high injection power of several $\mathrm{mW}$, a frequency band of at least $150 \mathrm{MHz}$ width is cleared from noise. As we have shown, this is sufficient for applying it to optical cooling of strontium atoms down to the mK level.

In our setup the master laser power available for injection was limited to $10 \mathrm{~mW}$, but we expect that even higher injection power will improve the slave laser stability, as has been shown by Y. Shimada et al. (43) using single-mode laser diodes. In future work, the TA SHG pro master laser will be replaced by a home-built ECDL laser in Littrow configuration operating with a Nichia NDB4216E-E laser diode delivering up to $40 \mathrm{~mW}$ of laser power, as it has been done in (43), resulting in a fully low cost system. 


\title{
6 Coherent backscattering of inelastic pho- tons from single atoms and their mirror im- ages ( $\mathrm{mCBS})$
}

\author{
Authors: P.H. Moriya ${ }^{\ddagger}$, R.F. Shiozaki ${ }^{\ddagger}$, R.C. Teixeira ${ }^{\ddagger}$, C.E. Máximo ${ }^{\ddagger}$, \\ N. Piovella* ${ }^{*}$ R. Bachelard ${ }^{\ddagger}$, R. Kaiser ${ }^{\dagger}$, Ph.W. Courteille
}

Same text of the paper that will be submitted to Scientific Journals.

Coherent backscattering is a coherence effect in the propagation of waves through disordered media involving two or more scatterers. Here, we report on the observation of coherent backscattering from individual atoms and their mirror images. This system displays two important advantages: First, the effect can be observed at low optical densities, which allows for example to work in very dilute clouds or far from resonance. Second, due to the fact that the radiation of an atom interferes constructively with that of its own image, the phenomenon is much more robust to dephasing induced by strong saturation. In particular, the contribution of inelastic photons to the interference process is demonstrated.

\subsection{Introduction}

Light propagating in an optically thick sample is subject to multiple scattering. Although part of the propagation can be explained by a diffusive model, interferences can alter the scattering. In particular, disorder in the sample may lead to an enhanced scattering into the backward direction $(14,45)$. The effect is known as coherent backscattering (CBS), and has first been studied with classical scatterers (46-49), and is interpreted as a precursor to the localization of light. The advent of laser-cooling techniques allowed to manipulate and control atomic gases, thus enabling their use as quantum scatterers. This triggered the study of coherent multiple scattering in a regime where the quantum internal structure, the wave-particle duality, and quantum statistical aspects play a role (50-53).

CBS is understood as resulting from the constructive interference between a scattering path involving two or more scatterers and the reciprocal (time-reversed) path. However, the quantum nature of the atoms leads to deviations in the behavior of CBS as compared to classical scatterers $(54,55)$. On one hand, the presence of a Zeeman structure can break the symmetry between the two reverse paths and reduces the contrast between the enhanced peak of radiation and the background (56). On the other hand, even a single

*Dipartimento de Fisica, Università degli Studi di Milano, Milano, Italy

${ }^{\dagger}$ Institut Non-Lineéaire de Nice, Valbonne, France

${ }^{\ddagger}$ Instituto de Física de São Carlos, Universidade de São Paulo, São Carlos, Brasil 
two-level atom will exhibit a non-classical behavior if strongly driven, and the scattering process becomes increasingly inelastic $(2,50,53,57)$. The corresponding phase fluctuations are potential sources for a loss of coherence and a reduction of the CBS contrast. Such saturation-induced loss of coherence in CBS was reported with a cold Strontium gas (1). Unfortunately, the theoretical treatment of saturation in multiple scattering is very challenging (58-65) and has not been solved in full generality.

Using a simple trick, coherence effects on the backscattered light can also be observed in optically thin samples, where multiple scattering is too weak to produce observable signatures: The introduction of a reflective interface - a dielectric mirror for example - allows for the radiation of the image scatterer to interfere constructively with that of the original scatterer, eventually resulting in a coherent backscattering process. This is a considerable advantage as compared to optically thick clouds, since it allows for a much easier theoretical analysis, e.g. of the contribution of inelastic photons (66).

This remaining m-CBS interference pattern stems from an inhomogeneous distribution of dipole populations within the atomic cloud, which in turn is a result of the standing wave created by the superposition of the incoming and the reflected probe beam. This spatial dipole population grating has no observable effects on the far-field scattering pattern, except at the specific directions $\mathbf{k}$ within the angular circle $\mathbf{k} . \hat{z}=-\mathbf{k}_{0} . \hat{z}$, where a simple calculation (see Suppl. Mat.; also Fig.1(b)) shows that all atoms at the maxima (minima) of the population grating will have a maximum (minimum) of their far-field interference fringes. The general intensity profile in the far-field presents then a circular symmetry around $\hat{z}$, as shown from a theoretical calculation in the top right area of Fig. 41. Because this set-up relies on a grating of the population of the dipoles, rather than of their phase, it shares similarities with other phenomena based on density gratings, such as the Classical Collective Atomic Recoil Laser (67) or some optomechanical instabilities (68); This differs from CBS, which relies on electronic (phase) grating, like Matter-Wave SuperRadiance (69) or Timed Dicke superradiant States (70). Also, the population grating appears to be quite robust to the phase decoherence usually induced by the saturation of the atoms, as it for example occurs in CBS.

(i\&ii)

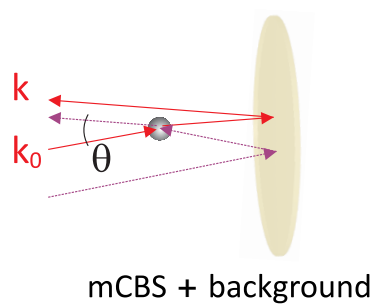

(iii)

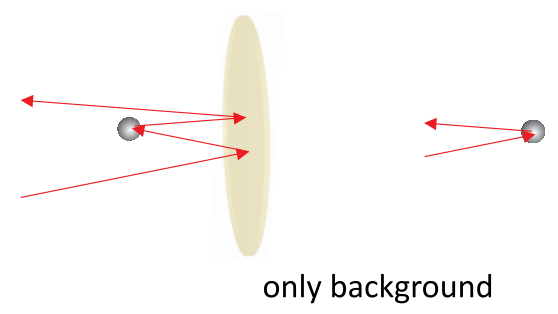

(iv)

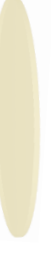

Figure 40 - Four processes exist in the single scattering regime, hereafter called (i-iv), depending on whether the incident light hits first the mirror or the atom, and whether it hits the mirror or not after being scattered.

By the author.

In this work, we report on the experimental observation of m-CBS from a lasercooled gas of Strontium atoms with a dielectric mirror. The series of circular fringes predicted by the theory are evidenced, the number and period of which allow to determine the cloud's position and longitudinal size. As one enters the strong field regime, the atoms get saturated and the comparison between theory and experimental results shows that, 
differently from CBS, inelastic photons do contribute to m-CBS.

\subsection{Experimental setup}

The setup for the m-CBS experiment is presented in Fig. 41(a) (see Sec. A of the Suppl. Mat. for details on the preparation of the cold Strontium sample). The scattering medium is a cold gas (temperature $\lesssim 10 \mathrm{mK}$ ) of ${ }^{88} \mathrm{Sr}$ atoms in its ground state ${ }^{1} S_{0}$, and the transition ${ }^{1} S_{0} \rightarrow{ }^{1} P_{1}$ (at $\lambda=2 \pi / k=461 \mathrm{~nm}$ with a linewidth of $\Gamma=(2 \pi) 30.5 \mathrm{MHz}$ ) is used for the resonant scattering. The $461 \mathrm{~nm}$ laser beam, called the m-CBS beam, has a waist of $1.5 \mathrm{~mm}$, is linearly polarized, and traverses a 50-50 non-polarizing beamsplitter before reaching the atoms a first time. A combination of two lenses with focal distance of $f=15 \mathrm{~cm}$ and separated by a distance of $2 f=30 \mathrm{~cm}$ creates a virtual image of a real mirror, placed at a distance $d$ after the last lens, at a distance $2 f-d$ before the first lens (68), as can be shown from a ray optics calculation (71). The m-CBS beam is reflected with an angle $\theta_{0} \sim 1^{\circ}$ compared to the mirror's normal direction, then crosses the cloud again before being partially reflected by the beamsplitter onto a $200 \mathrm{~mm}$ lens. The angular radiation pattern impinging at the lens is converted at its focal plane into a spatial one, where a CCD camera is placed such as to capture part of the circular pattern determined by the condition $\mathbf{k}_{0} \cdot \hat{z}=-\mathbf{k} \cdot \hat{z}$, while avoiding the direction of the reflected $\mathrm{m}$-CBS beam (which lies also within the circle) in order not to saturate its pixels.

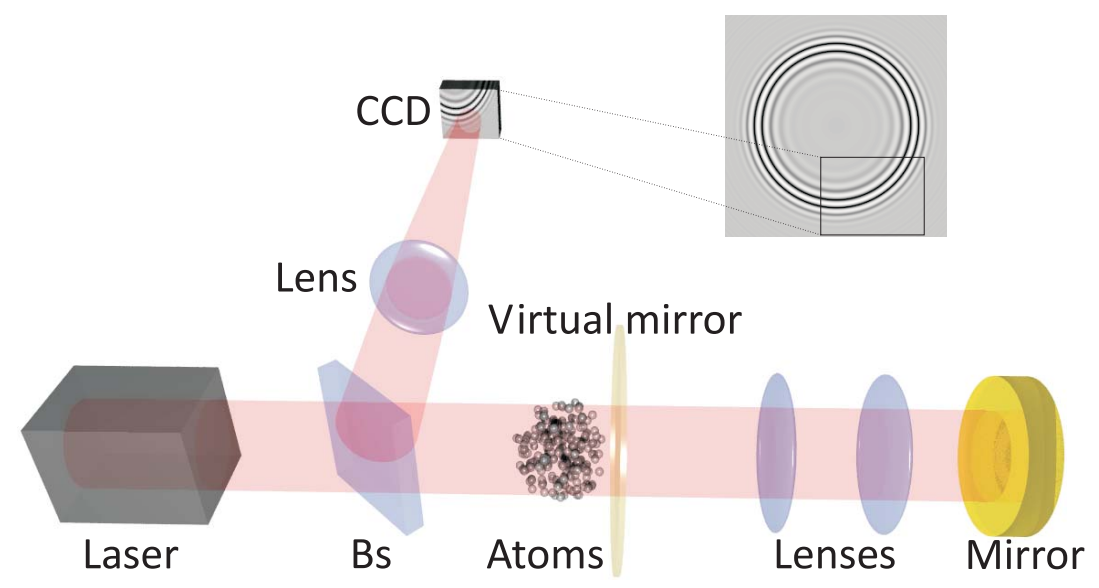

Figure 41 - Experimental setup: A laser beam passes through a polarizer (Pol) and a wedged beamsplitter (BS) before passing through the atomic cloud a first time. It is then reflected on a virtual mirror $(\mathrm{VM})$ created through two further lenses and a physical mirror at a small angle of $\theta_{0} \sim 1^{\circ}$ with the normal of the mirror. After being reflected, it crosses the cloud again, before being sent by the beamsplitter, together with the light scattered from the atoms, to a CCD camera that images the angular distribution of the light. The top right picture stands for the theoretical fringe pattern for the mCBS set-up, see Suppl. Mat.

By the author.

During an experimental sequence, a MOT of $10^{8}$ atoms and $1 / \sqrt{e}$ radius of $0.9(1) \mathrm{mm}$ is loaded within $2 \mathrm{~s}$. $200 \mu \mathrm{s}$ after turning off the MOT cooling beams we shine a m-CBS beam pulse of $200 \mu$ s onto the atomic cloud, capturing an image in presence of atoms. In order to account for the stray light created by the reflected m-CBS beam that reaches the 
camera, after 1s we register a second image in absence of atoms, to be subtracted from the first one. We run this sequence at least 200 times for the same parameters to obtain a disorder-averaged final image as shown in Fig.42(a).

\subsection{Linear regime}

The interference phenomenon of $\mathrm{m}$-CBS is best understood in the linear regime (i.e., for a saturation parameter $s \ll 1$ ), when the atoms behave as classical scatterers. In this limit, the radiation of a Gaussian cloud of atoms and its mirror image, measured at an angle $\theta$ to the normal of the mirror, reads (see Suppl. Mat.)

$$
\frac{I(\theta)}{I_{0}} \approx s\left[1+\frac{1}{2} e^{-2\left(\theta_{0} k \sigma_{z}\right)^{2}\left(\theta-\theta_{0}\right)^{2}} \cos \left(2 \theta_{0} k h\left(\theta-\theta_{0}\right)\right)\right],
$$

where $h$ is the distance between the (virtual) mirror and the center of the atomic cloud, $\sigma_{z}$ is the longitudinal cloud radius at $1 / \sqrt{e}, s=2 \Omega_{0}^{2} /\left(\Delta^{2}+\Gamma^{2} / 4\right)$ the off-resonant saturation parameter, with $\Omega_{0}$ the Rabi frequency of the laser and $\Delta$ the laser detuning from the atomic resonance; $s I_{0}$ corresponds to the background radiation. For obtaining the above equation, a small angle approximation has been applied $\left(\theta_{0} \ll 1\right.$ and $\left.\left|\theta-\theta_{0}\right| \ll \theta_{0}\right)$. The second term in the bracket corresponds to the single scattering interferences, still present after averaging on the atomic spatial Gaussian distribution within a Gaussian angular envelope of half-width at $1 / e^{2}$ given by $\Phi=1 / \theta_{0} k \sigma_{z}$. Since the fringes have an angular period $\Theta_{f}=\pi / \theta_{0} k h$, one typically expects to observe a number $\sim h / \pi \sigma_{z}$ of fringes on the scattered light.

We realize the first set of measurements with a saturation parameter $s=0.2$ (at the center of the Gaussian beam). A typical interference pattern is shown in Fig. 42(a). After determining the center of the interference rings we perform an azimuthal average and obtain an interference pattern (see inset of Fig. 42(a)). The fact that the reflected beam is attenuated after the passage through the cloud results in extra stray light in the background image as compared to the image taken in presence of the atoms. Thus, we remove this slowly varying envelope before fitting the interference pattern to Eq. (6.1) using the cloud size $\sigma_{z}$, the fringes' period $\Theta_{f}$ and the amplitude of the main interference peak as fitting parameters.

The (virtual) mirror position $h$ was varied for about $6 \mathrm{~cm}$ around the center of the cloud, and for each position an interference pattern similar to that of Fig. 42(a) was extracted. Note that we are able to place the virtual mirror at negative distances (i.e. the light first passes through the virtual mirror and then the atoms), and still have the m-CBS effect. Fig.42(b) shows the measured dependence of the fringes' period $\Theta_{f}$ (or, equivalently, of the deduced mirror distance $\left.\pi / k \theta_{0} \Theta_{f}\right)$ as a function of $h$. The excellent linear behavior not only shows a good agreement with theory, but also indicates that the initial experimental positioning was misaligned by 2.1(1) $\mathrm{mm}$.

\subsection{Saturated regime}

CBS is strongly affected by saturation, with a drastic reduction of the contrast as the saturation parameter goes above unity (1). Indeed the system enters the inelastic 

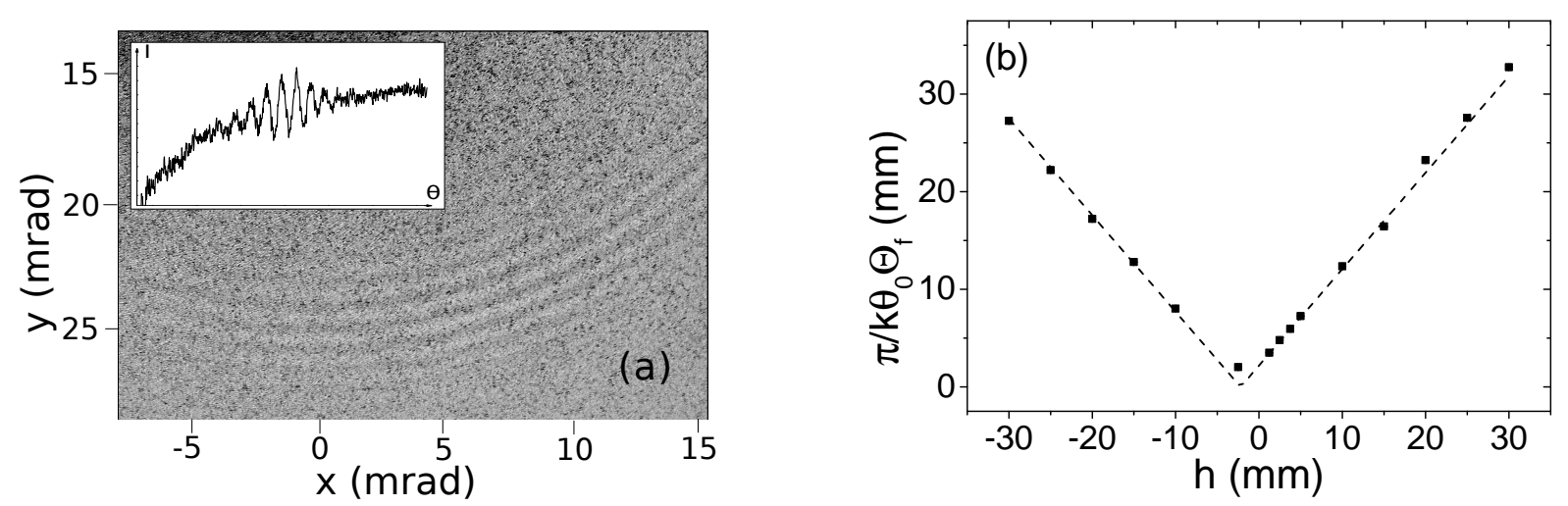

Figure 42 - (a) Averaged image collected on the CCD, after substraction of the background, showing the interference pattern due to the mCBS effect. Inset: fringes' pattern obtained from azimuthal averaging. (b) Mirror position expected from mCBS theory (i.e., $h_{t h r}=p i / k \theta_{0} \Theta_{f}$ ) as a function of the experimental mirror position. This measurement allowed to detect an initial experimental mispositioning of $x_{0}=2.2(1) \mathrm{mm}$, which corresponds to the shifted minimum of the fit $h_{t h r}=A h+x_{0}$ (dashed line); we obtained $A=0.988(12)$.

By the author.

scattering regime where the broadening of the fluorescence spectrum (e.g., Mollow triplet) results in a loss of phase coherence of the scattered waves. In addition a precise theory is challenging, in particular due to the geometry of the system used since finite optical thicknesses imply that the cloud is not homogeneously excited.

In the m-CBS set-up, since the coherent emission relies on the population grating rather than phase coherence of the scatterers, the main mechanism responsible for the extinction of the contrast with the saturation is expected to be the disappearance of the dipole population grating. Indeed, as the saturation parameter $s$ increases the dipole population differences becomes less and less pronounced, and in the limit of high $s$ all atoms become equally excited (i.e., they are saturated), and thus the cloud radiates isotropically. From a theoretical point of view, we have the important advantage of considering a single scattering process, which allows to deal with complex geometries.

While the analysis in the linear regime focused on the period and envelope of the fringes, a precise measurement of the contrast and its dependence on the saturation parameter requires a careful treatment of the stray light. As mentioned previously, the reflected stray light is stronger without the atoms than in their presence, since it is then partially absorbed by the cloud. It is also strongly inhomogeneous (see Fig.42(a)). Hence a simple image subtraction will not eliminate the stray light background and is not sufficient for an accurate determination of the fringes contrast. The treatment of the raw data to account for the absorption of the stray light by the atoms is detailed in the Suppl. Mat.

For a given mirror position $(h=8.0(5) \mathrm{mm})$ and cloud size $\left(\sigma_{z}=0.9(1) \mathrm{mm}\right)$, we have repeated the experimental sequence described before for several different intensities of the m-CBS beam. This allowed us to obtain a series of curves from which the absolute fringes contrast could be extracted. Fig. 43 confronts the saturation behavior of the enhancement factor measured in our m-CBS setup and conventional CBS measured by other groups, as a function of the saturation parameter $s$ at the center of the m-CBS beam, plus two theoretical predictions that we shall discuss below. Note that we here define the 
contrast from the atomic fluorescence $I_{f}(\theta)$ as $\left(I_{f}^{\max }-I_{f}^{\min }\right) /\left(I_{\text {background }}\right)$, for both CBS and $\mathrm{m}-\mathrm{CBS}$. The CBS data presented here refer to an experiment realized with Strontium atoms(1), with a probe beam at resonance, and they exhibit almost the maximum CBS contrast of 1 in the linear regime. CBS results were also obtained with Rubidium atoms which, due to their non-trivial internal structure, exhibit an overall lower contrast, even at low saturation parameter (50). Note that in order to observe CBS, a high optical thickness $(b>1)$ is necessary to reach a significant contrast, whereas in the m-CBS set-up, the maximum contrast is achieved at low optical densities.

The first striking result is that while the contrast decreases quickly for CBS as the saturation parameter is increased, the m-CBS phenomenon is quite robust to saturation (it stays between $\sim 0.4$ and $\sim 0.5$ from the linear to the $s=20$ regime). To understand this observation, we discriminated in the theory the elastic and inelastic photons, as the latter starts to dominate the scattering process as the saturation parameter increases. The "elastic theory" in Fig.43 refers to the m-CBS contrast assuming that only the elastic photons contribute to the interference, whereas the background is computed summing both the elastic and inelastic light (see Suppl. Mat.). The resulting curve appears to drop quickly for increasing saturation, at odds from the experimental m-CBS data, but in a way similar to the CBS. It is known that CBS, that involves two non-correlated atoms (in contrast to an atom and its mirror image), is strongly affected by inelastic scattering $(1,2,58,59,61,64,65)$.

Assuming now that all photons contribute to the interferences, including the ones inelastically scattered (as those of the Mollow triplet), we derived the interference pattern and its contrast for the m-CBS set-up (see Suppl. Mat.). In other words, both elastic and inelastic scattering contribute to both the fringes and the background, and the resulting contrast corresponds to the upper gray-shaded area in Fig.43: It presents the same slow decay, as a function of the saturation, as the experimental data. Both theoretical curves take into account the finite size effects of our Gaussian beam and our Gaussian atomic cloud, that was verified not to affect substantially the main dependence on $s$.

The quasi-absence of decay of the contrast observed experimentally is a strong argument in favor of a participation of the inelastic scattering to the m-CBS interference process. There is a remaining discrepancy between theory and experiment, that may be explained in at least two ways: First, the small optical thickness of our atomic cloud ( $b \sim 0.6$ at its center) implies that, due to absorption (not accounted for in our theory), the processes (i)-(iv) described in Fig.?? do not contribute with the same amplitude anymore. The fact that the transmission tends to unity at large saturation (see Fig.44(b) from Suppl. Mat.) agrees with the observation that the discrepancy between theory and experiment reduces at larger saturation. Second, our theory assumed an absence of dephasing for inelastic photons, i.e., they contribute as much to the interference as the elastically scattered ones. A more involved approach would require, for example, diagrammatic approaches, such as those developed for CBS.

\subsection{Conclusions}

In conclusion, we have observed coherent backscattering from a cloud of cold atoms and its mirror image. This set-up was shown to provide a measurement of the cloud longitudinal size and position with a precision of $100 \mu \mathrm{m}$. Investigating the saturated regime, 


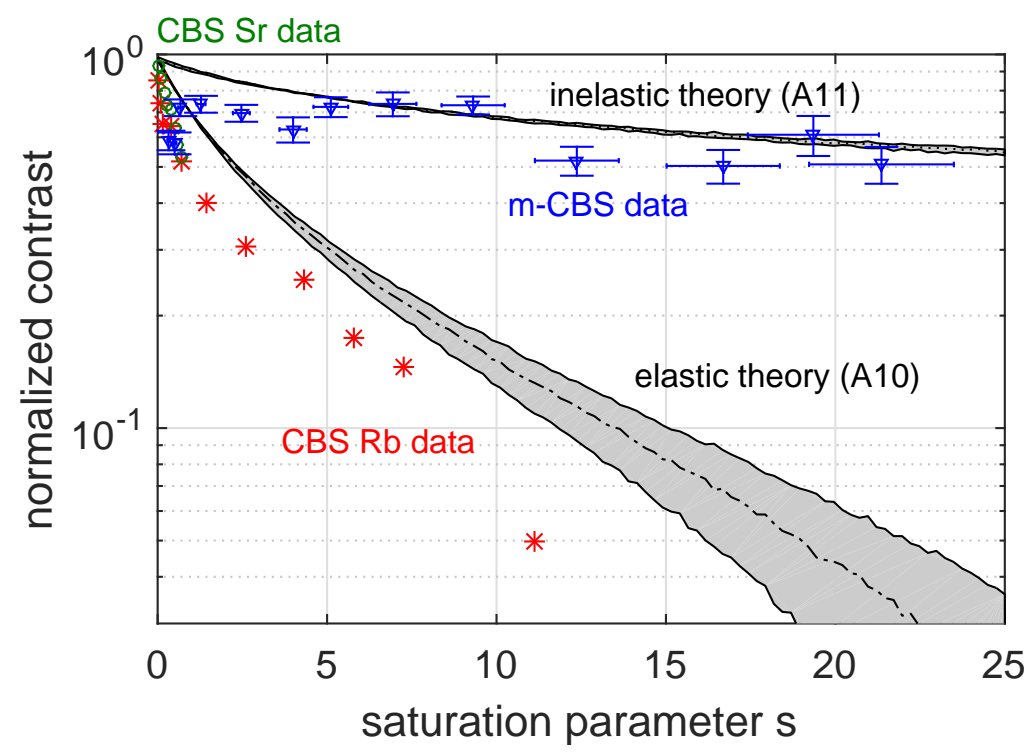

Figure 43 - Experimental mCBS contrast (blue triangles), compared to the CBS one from a Strontium cloud (1) (green circles) and from a Rubidium cloud (2) (red stars). The gray areas for the theoretical predictions (for the elastic and inelastic theories (see main text and Suppl. Mat.)) account for the precision within which the experimental parameters are known: $h=8 \pm 0.05 \mathrm{~mm}$ and $\sigma_{z}=0.85 \pm 0.1 \mathrm{~mm}$. The data are a function of the saturation parameter $s$ at the center of the probe beam. The Rb contrast was renormalized so it is 1 in the linear regime, despite the multilevel structure of the atoms.

By the author.

we could identify the important contribution of inelastic photons to the interference process, at odds from CBS. Because this coherence effect appears in completely different regimes as compared to CBS, such as low optical densities and high saturation parameters, it can represent a very important tool for probing coherences in the atomic scattered light where CBS is not anymore observable. In particular, the atom and its mirror image are strongly correlated so it could be used to probe non-classical light effects $(72,73)$.

Acknowledgements. - We appreciate helpful insights from Thibaut Jonckheere and Dominique Delande. We thank William Guérin for his critical reading of the manuscript. This work has received financial support from FAPESP, CNPq (PVE 400228/2014-9) and CAPES Brazilian agencies.

\subsection{Supplementary material}

\subsubsection{Preparation of the Strontium cold cloud}

We prepare our atomic sample in a typical Strontium apparatus, which we briefly describe in the following. A collimated atomic beam emerges from an array of microtubes located at the output of an oven heated to $550^{\circ} \mathrm{C}$. The atomic beam is then decelerated in a $28 \mathrm{~cm}$ long Zeeman Slower in spin-flip configuration by a counterpropagating laser beam of power $\sim 40 \mathrm{~mW}$ tuned $500 \mathrm{MHz}$ to the red of the $461 \mathrm{~nm}$ resonance. The Zeeman 
Chapter 6. Coherent backscattering of inelastic photons from single atoms and their mirror images 82

$(m C B S)$

slower beam has a $1 / e^{2}$ radius of $4 \mathrm{~mm}$ at the entrance of the experimental apparatus, being focused onto the oven output after propagation through the whole system (about $90 \mathrm{~cm}$ long). The cooled Strontium beam is captured in the science chamber by a magnetooptical trap (MOT) generated by three pairs of counter-propagating collimated $461 \mathrm{~nm}$ laser beams and a quadrupole magnetic field; the latter is produced by a pair of coils in anti-Helmholtz configuration (axial magnetic gradient $|\nabla B|=70 \mathrm{G} / \mathrm{cm}$ ). Each laser beam has a $1 / e^{2}$ radius of $5 \mathrm{~mm}$ and is detuned by $-40 \mathrm{MHz}$ from resonance. A repumping laser addressing the $497 \mathrm{~nm}{ }^{3} P_{2} \rightarrow{ }^{3} D_{2}$ transition is used to recycle atoms that have decayed to the metastable state ${ }^{3} P_{2}$. In this way, we are able to generate cold gases with $\sim 10^{8}{ }^{88} \mathrm{Sr}$ atoms at a temperature below $10 \mathrm{mK}$. Resonant absorption imaging reveals an approximate Gaussian density profile with a $1 / \sqrt{e}$ radius of $0.9(1) \mathrm{mm}$.

\subsubsection{Data analysis: subtraction of the stray light}

As described in the main text, at each experimental run we obtain two images: The first one is registered while the m-CBS beam impinges on the atomic cloud, and the second one is done in the same conditions, but with no atoms captured in our trap. The m-CBS reflected beam, after traversing all optical elements, creates an angular stray light profile on directions close to its propagation direction. The image registered in absence of atoms shows exclusively this stray light pattern; The image with atoms has this pattern superposed to the atomic fluorescence, that we want to extract. All the difficulty in extracting it stems from the fact that when the atoms are also present, the m-CBS beam is partially absorbed by them, and creates a smaller effect on the camera when compared to its effect without absorption. We can thus write the azimuthal-averaged profile of the light intensity in presence of atoms as

$$
I_{a}(\theta)=T I_{s t}(\theta)+I_{f}(\theta)
$$

where $T$ stands for the average transmission of the cloud after the double passage of the reflected m-CBS beam, and $I_{s t}$ and $I_{f}$ for, respectively, the intensity of the stray light in the absence of atoms, and the intensity of the atomic fluorescence only. From $I_{f}(\theta)$, the absolute contrast can thus be extracted. Out of the fringes' envelope (or, to a good approximation, for $\left.\left|\theta-\theta_{0}\right|>2 \Phi\right)$, and since we are monitoring the intensity in a narrow angle of $10 \mathrm{mrad}$, the fluorescence background is isotropic to an excellent approximation. We can write then

$$
I_{a}\left(\theta,\left|\theta-\theta_{0}\right|>2 \Phi\right)=T I_{s t}(\theta)+I_{b g},
$$

where $I_{b g}$ is the constant incoherent atomic background. Then, the stray light profile is determined by finding the linear combination of $I_{a}(\theta)$ (measured with the atoms) and $I_{s t}(\theta)$ (measured without the atoms) that satisfies (6.3) outside of the fringes region. An example is provided in Fig.44(a), where the measured intensity profile with and without the atoms is presented, as well as the extracted atomic fluorescence. Figs.44(b) and (c) show the fitted parameters $T$ and $I_{b g}$, respectively, as a function of the saturation parameter $s$ at the center of the atomic beam (black circles). The dashed lines correspond to a simple model for the interaction between our saturated Gaussian laser beam and our Gaussian shaped atomic cloud, with the optical density at the center as the only free parameter. The good agreement gives us confidence in the fitting procedure for extracting the pure atomic fluorescence out of our raw data. 

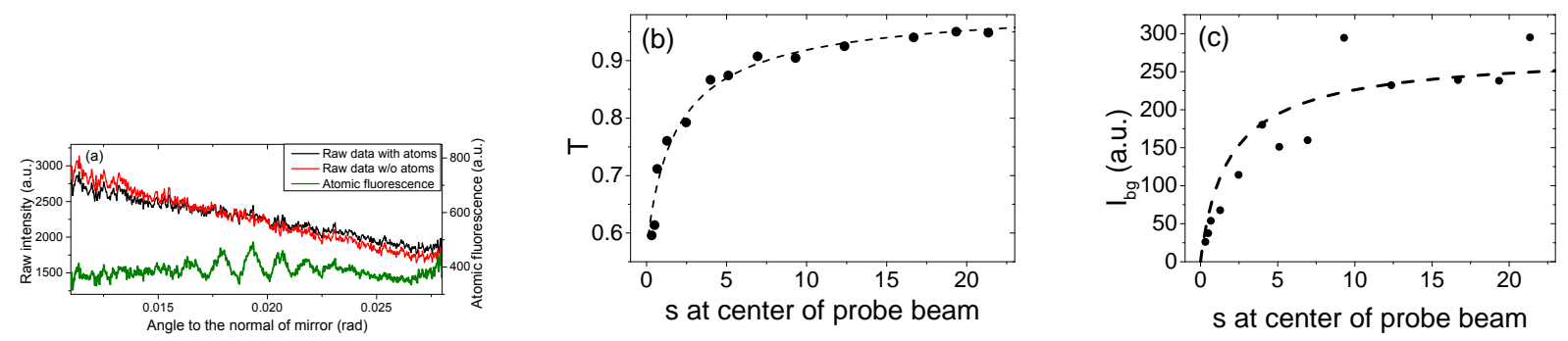

Figure 44 - (a) Angular intensity profile in presence (middle black curve) and in absence (top red curve) of the atomic cloud. The deduced atomic fluorescence after the stray light weighted subtraction (see text) $I_{f}(\theta)$ (lower green curve, right vertical axis) presents a locally isotropic background, plus an interference pattern at the center. (b) Transmission coefficient $T$ and (c) background intensity $I_{b g}$ deduced from the fit (see Eq.(6.3) and main text), as a function of the on-axis saturation parameter $s$. The dashed curves are calculated with a simple model for the interaction between our saturated Gaussian laser beam and our Gaussian atomic cloud.

\subsubsection{Single-scattering theory}

In the following we outline the theoretical approach used to obtain the radiated intensity pattern in the linear and saturated regimes. Since we are focusing on the single scattering regime, it is sufficient to study the behaviour of single atoms, and then sum coherently their radiation, i.e., their electric field. Let us thus consider a two-level atom at position $\mathbf{r}=(x, y, z)$ driven by a field of wavevector $\mathbf{k}_{0}=k\left(0, \sin \theta_{0},-\cos \theta_{0}\right)$.

Without loss of generality, we assume that the (virtual) dielectric mirror lies in the plane $z=0$ and choose the initial polarization along the $\hat{\mathbf{x}}$ axis. The incident field plus its mirror generate a standing wave in the $z$-axis and a propagating one in the $y$ direction:

$$
\Omega(\mathbf{r})=\Omega_{0} \cos \left(k z \cos \theta_{0}\right) e^{-i k y \sin \theta_{0}} .
$$

Note that we have here assumed high quality mirrors that possess unity reflection coefficients.

The single-atom equations are then used to determine the radiation of each atom. Calling $\sigma, \sigma^{\dagger}$ and $\sigma^{z}$ the atomic operators, in the semiclassical limit the atomic dynamics is given by the following set of equations (74):

$$
\begin{aligned}
\frac{d \hat{\sigma}}{d t} & =\left(i \Delta-\frac{\Gamma}{2}\right) \hat{\sigma}+i \Omega(\mathbf{r}) \hat{\sigma}_{z}, \\
\frac{d \hat{\sigma}^{z}}{d t} & =i\left(\Omega^{*}(\mathbf{r}) \hat{\sigma}-\Omega(\mathbf{r}) \hat{\sigma}^{\dagger}\right)-\Gamma\left(\hat{\sigma}^{z}+1\right),
\end{aligned}
$$

with the commutation relations $\left[\hat{\sigma}, \hat{\sigma}^{z}\right]=2 \hat{\sigma},\left[\hat{\sigma}^{\dagger}, \hat{\sigma}\right]=\hat{\sigma}^{z}$ and $\hat{\sigma}^{z} \hat{\sigma}=-\hat{\sigma}$.

By imposing the temporal derivatives to be zero, one obtains the steady state $(s s)$ expectation values of the optical coherence and excited population for an atom at position r:

$$
\begin{aligned}
\langle\hat{\sigma}\rangle_{s s}(\mathbf{r}) & =\frac{\Delta^{2}+\Gamma^{2} / 4}{\Delta+i \Gamma / 2} \frac{2 \Omega(\mathbf{r})}{\Delta^{2}+\Gamma^{2} / 4+|\Omega(\mathbf{r})|^{2}}, \\
\left\langle\hat{\sigma}^{\dagger} \hat{\sigma}\right\rangle_{s s}(\mathbf{r}) & =\frac{|\Omega(\mathbf{r})|^{2}}{2\left(\Delta^{2}+\Gamma^{2} / 4+|\Omega(\mathbf{r})|^{2}\right)} .
\end{aligned}
$$


Chapter 6. Coherent backscattering of inelastic photons from single atoms and their mirror images 84

$(m C B S)$

In the far field limit, the field radiated by a single atom in a direction $\mathbf{k}=k \hat{\mathbf{n}}$ and at a distance $r$ reads (75)

$$
\hat{\mathbf{E}}(\mathbf{k}, t)=-\hat{\sigma}(t) \frac{d k^{2}}{4 \pi \epsilon_{0} r}[\hat{\mathbf{n}}(\hat{\mathbf{x}} \cdot \hat{\mathbf{n}})-\hat{\mathbf{x}}] e^{-i \mathbf{k} \cdot \mathbf{r}},
$$

where $d$ refers to the dipole coupling element, $\epsilon_{0}$ to the vacuum permittivity.

The measured field from the m-CBS experiment will actually contain two contributions from each atom, since the radiation of the latter may be reflected or not by the mirror (see Fig.41(c), with processes (i) and (iv) that yield mirror reflection after scattering). Thus the total scattered electric field $\mathbf{E}_{s}$ in a direction $\mathbf{k}$ comes from the superposition

$$
\mathbf{E}_{s}(\mathbf{k})=\mathbf{E}\left(k_{x}, k_{y}, k_{z}\right)+\mathbf{E}\left(k_{x}, k_{y},-k_{z}\right) .
$$

In general, polarization and the different components of the field may play an important role in the intensity profile. However our experiment was carried out within observation angles $\theta \ll 1$, where only the $E_{x}$ component is significant. Hence we obtain for the steadystate fields the following expression:

$$
\begin{aligned}
\left\langle E_{s}\right\rangle & \sim \sqrt{C}\langle\sigma\rangle_{s s} \cos (k z \cos \theta) e^{-i k_{y} y-i k_{x} x}, \\
\left\langle E_{s}^{\dagger} E_{s}\right\rangle & \sim C\left\langle\sigma^{\dagger} \sigma\right\rangle_{s s} \cos ^{2}(k z \cos \theta),
\end{aligned}
$$

where the prefactor $C=d^{2} k^{4} /\left(4 \pi^{2} \epsilon_{0}^{2} r^{2}\right)$ is unimportant to the determination of the contrast. Eq.(6.11) corresponds to the optical coherence, and thus to the elastically scattered light. On the contrary Eq.(6.12) describes the total intensity, that is, both elastic and inleastic photons (74).

Moving to the m-CBS by a macroscopic cloud of $N$ atoms with positions $\mathbf{r}_{j}$, the radiation pattern (resp. elastic and total) is computed from the superposition of all atom independent radiations:

$$
\begin{aligned}
\frac{I_{\text {ela }}}{I_{0}} & =\frac{16 s}{N} \sum_{j=1}^{N} \frac{\cos ^{2}\left(k z_{j} \cos \theta_{0}\right) \cos ^{2}\left(k z_{j} \cos \theta\right)}{\left(2+s \cos ^{2}\left(k z_{j} \cos \theta_{0}\right)\right)^{2}} \\
\frac{I_{\text {tot }}}{I_{0}} & =\frac{8 s}{N} \sum_{j=1}^{N} \frac{\cos ^{2}\left(k z_{j} \cos \theta_{0}\right) \cos ^{2}\left(k z_{j} \cos \theta\right)}{2+s \cos ^{2}\left(k z_{j} \cos \theta_{0}\right)}
\end{aligned}
$$

where we have introduced the saturation parameter $s=2 \Omega_{0}^{2} /\left(\Delta^{2}+\Gamma^{2} / 4\right)$ and $I_{0}=$ $c \epsilon_{0} N C / 16$. Eq.(6.14) relies on the simplifying hypothesis that all the scattered light (elastic and inelastic) has the same phase after the scattering, at any time. We note that, in the case of a uniform intensity distribution of the laser beam, the spatial distribution of the atoms in the plane of the mirror $(x, y)$ does not play any role in this set-up.

The linear regime, which is that of elastic scattering $\left(I_{\text {ela }} \approx I_{\text {tot }}\right)$, is obtained by taking the $s \ll 1$ limit. We obtain the following expression for the microscopic system:

$$
\frac{I_{\text {ela }}(\theta)}{I_{0}} \approx \frac{8 s}{N} \sum_{j=1}^{N} \cos ^{2}\left(k z_{j} \cos \theta_{0}\right) \cos ^{2}\left(k z_{j} \cos \theta\right),
$$

The macroscopic behaviour is captured by substituting the sum over the atoms by an integral over the typical atomic distribution $\sum_{j=1}^{N} \rightarrow \int \mathrm{d} \mathbf{r} \rho(\mathbf{r})$. For a Gaussian distribution such as those produced in our trap, one obtains Eq.(6.1). That equation predicts an 

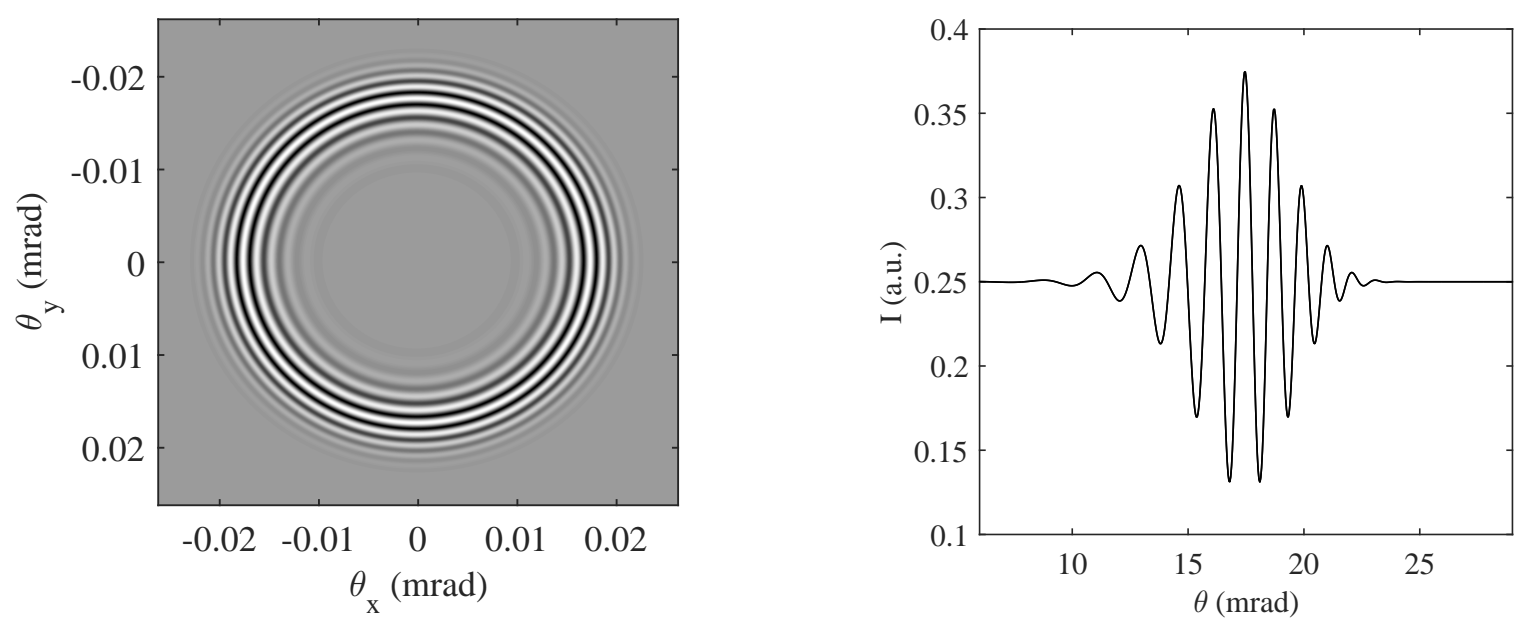

Figure 45 - (a) Intensity pattern in the elastic regime (6.13). (b) Azimuthal profile of Fig.(a), showing the fringes profile. The figures can be compared to the measurement presented in Fig. 42(b).

alternation of constructive and destructive interferences with period $\pi /\left(k h \theta_{0}\right)$, which leads to the observed fringes, see Fig. 45.

Remark that the elastic contribution Eq.(6.13) decreases as $1 / s$ for increasing saturation parameter $s$, whereas the total radiation converges to:

$$
\lim _{s \rightarrow \infty} \frac{I_{t o t}(\theta)}{I_{0}}=\frac{8}{N} \sum_{j=1}^{N} \cos ^{2}\left(k z_{j} \cos \theta\right) .
$$

Integrating over a Gaussian distribution as before, in the small angle and large cloud limits, we obtain $I_{t o t} / I_{0}=1 / 2$, i.e., the fluorescence converges to a finite value for very large saturation parameters. Thus the ratio between the elastically scattered intensity to the total one scales as $1 / s$, which explains the fast decay of the contrast of the 'elastic' theory.

In the saturated regime, atoms that are not close to a zero of the intensity of the created standing wave saturate. As $s$ increases, the proportion of scatterers that contribute to the grating decreases as $1 / \sqrt{s}$, whereas the others produce an isotropic fluorescence radiation pattern. This explains the rather slow decay of the contrast in the 'inelastic theory'. In the present work, the general intensity pattern for $s>1$, but finite, was computed numerically using the microscopic formula (6.14), for random Gaussian distributions of millions of atoms (see Fig.43). 



\section{Outlook on the next experiments and the future of the experiment}

The long-term goal of our strontium experiment is to produce cold, ultracold and degenerate samples of strontium, ordered (optical lattices) and disordered (magneto optical traps and conservative traps), for the study of collective effects in light scattering. In the next sections, some near future experiments are presented as well as the next generation of our apparatus.

\subsection{Collective effects experiments}

Cold bosonic strontium clouds presents an alternative to traditional alkaline systems for the study of collective phenomena in light scattering, due to its unique cooling transitions and its non-magnetic ground level.

As an example, it is possible to show that sufficiently cold atomic samples exposed to a gradient of inhomogeneous magnetic field exploiting the Zeeman effect, deflect light due to collective scattering in the single-scattering regime (as shown in Fig.46). The deflection is a result of the interference of the light radiated by independent atoms. This effect was named atomic Lighthouse effect, due to its similarity to a nuclear physics phenomena. In the single scattering regime(76), the maximum deflection angle is equal to

$$
\theta_{L}=\arcsin \left\{\frac{1-\sqrt{1+4 \alpha^{2} \sigma^{2}}}{2 \alpha \sigma^{2}}\right\} \approx-\alpha=\frac{2 \mu_{B} b}{\hbar k_{0} \Gamma}
$$

where $\mu_{B}$ is the Bohr magneton, $b$ is a linear magnetic field, $k_{0}$ is the laser beam wave vector and $\Gamma$ is the transition linewidth. For strontium in the broad blue and narrow red transition the deflected angle is expected to be equal to $0.03^{\circ}$ and $1^{\circ}$, respectively, for typical experimental parameters.

Other possibility is the experimental study of the modification in the radiation pressure by cooperative scattering. (77) have proposed that: "cooperative spontaneous emission of a single photon from a cloud of $\mathrm{N}$ atoms modifies substantially the radiation pressure exerted by a far-detuned laser beam exciting the atoms. On one hand, the force induced by photon absorption depends on the collective decay rate of the excited atomic state. On the other hand, directional spontaneous emission counteracts the recoil induced by the absorption. It has been shown that the radiation pressure depends on the atom number via cooperative scattering and that, for certain atom numbers, it can be suppressed or enhanced. Cooperative scattering of light by extended atomic clouds can become important in the presence of quasi-resonant light and could be addressed in many cold atoms experiments". 


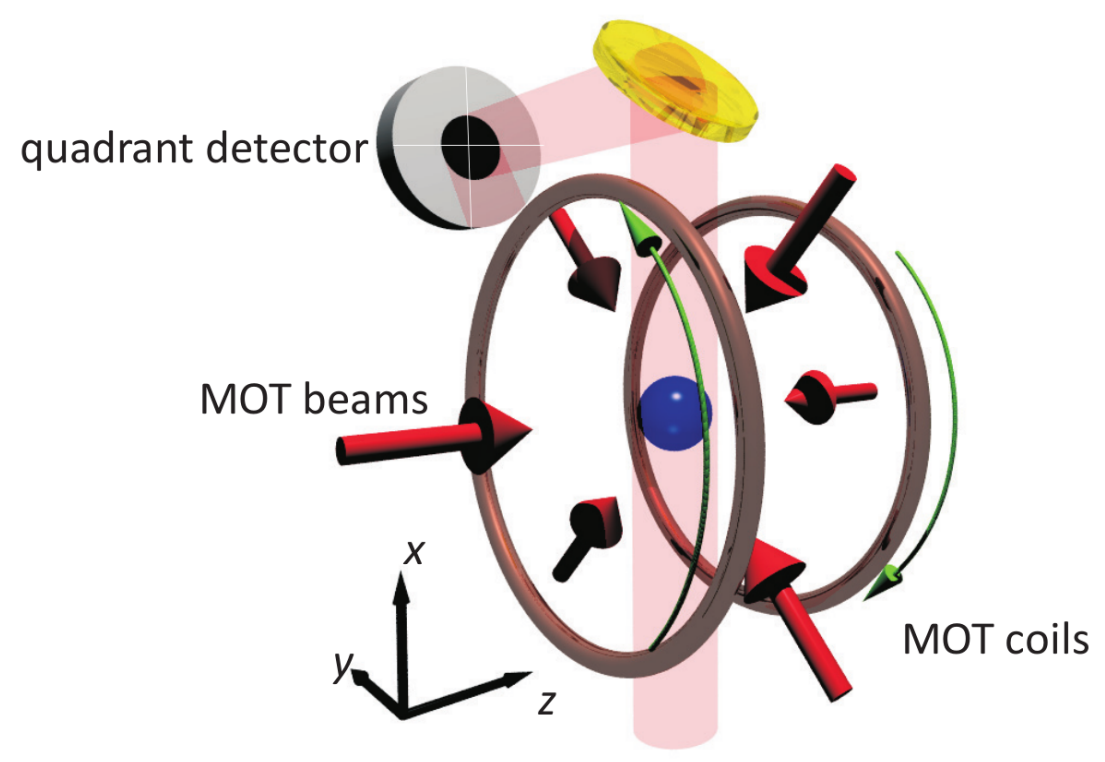

Figure 46 - Experimental setup for lighthouse effect experiment. The quadrant detector is used because it can measure any vertical and horizontal small displacements.

By the author.

\subsection{Experiment next generation}

Despite all the improvement done since the experiment started to produce cold and ultracold samples, as pointed in the previous sections, the current experimental apparatus possibly will not produce quantum degenerate samples and optical lattices. The main reason is the ultra-high vaccum which needs a reduction of at least 2 orders of magnitude, reaching a ideal value of $10^{-12}$ mbar. We expect to reach it by decreasing the total volume of the system and better extraction of impurities.

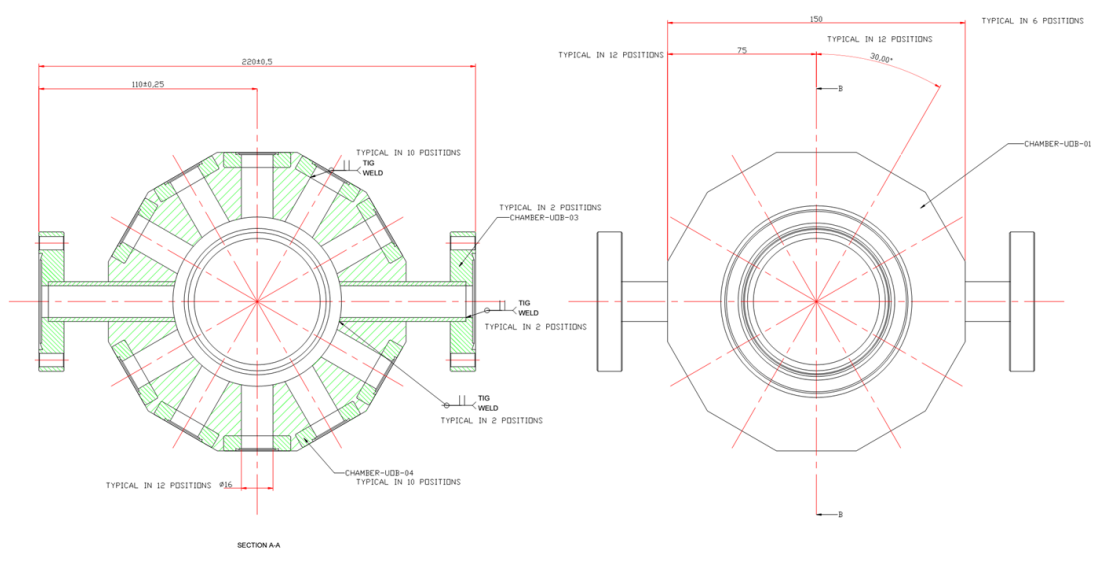

Figure 47 - New dodecagon-shaped chamber for the next generation of the experiment.

By the author.

The central part of the new apparatus is a dodecagon chamber (see Fig. 47) designed by a specialist vacuum company. The windows will have improved anti-reflective coating (reflectivity below $0.5 \%$ ) and they are directly bonded to the chamber. As the 
name says, it has 12 sides, which includes 10 CF16 viewports and two CF35 flanges to connect the science chamber to other parts of the experiment. In its axial sides two reentrant CF63 viewports are installed. The total thickness axial is $15 \mathrm{~mm}$ which will allow the coils to be closer to each other and to the atoms. This configuration will decrease the dimensions of our MOT coils and, consequently, reduce the size and the switch off time of the coils.

Other parts of the experiment are under development, based on the size of the new science chamber. For example, a new version of the Zeeman slower is under development. Recently, more options based on permanent magnets, instead of the traditional coils, have been published $(78,79)$ which present better stability and are easily tunable. New quadrupole coils, and compensation Earth and spurious background magnetic field coils will be developed as well. In the oven section, our main concern is to decrease the atomic beam divergence angle to few mili-radians and to provide a better heating system to the microtubes in order to avoid clogging.

\subsection{Optical dipole trap, Bose-Einstein condesates and Optical lat- tices}

As mentioned above, this strontium experiments aims to reach ultracold samples of strontium and Bose-Einstein condensates to transfer them to 1D, 2D and 3D optical lattices. More information about this topics can be found in the Appendix.

After the stabilization of the magneto-optical trap in the strontium narrow transition, an optical dipole trap will be created. For this trap, the general idea is to use an 25 W, $1064 \mathrm{~nm}$ Mephisto MOPA (Coherent Inc.). After this stage, all the hot atoms will be evaporated using the evaporative cooling technique. With an ultracold sample in hands, a Bose-Einstein condensate can be created or the atoms can be directly transferred to 1D and 2D optical lattices.

Those results can be extended to two and three dimensions. Due to its favorable electronic structure, is predicted for strontium(80) the possibility to reach exotic lattices, such as diamond-like structures, and the presence of photonic bandgaps. 



\section{Conclusions}

In the last 4 years, we stabilized our current experimental setup. First, I worked on the MOT coils feedback loop control electronics to control and stabilize the quadrupole magnetic field by controling the current applied in each coil. With this improvement, we could speed up the magnetic field rise and fall time from $10 \mathrm{~ms}$ to $2 \mathrm{~ms}$, limited only by the coils size which limits important parameters such as resistance and inductance. For the next generation, a better science chamber geometry will allow us to decrease its size and response time.

We also developed as well a new generation of MOT coils, since we started to notice short circuits between the layers and raise of temperature after few experimental cycles. In the new generation, we used acetal resin to construct a housing which permits the coil to be in direct contact with water maintained at $15^{\circ} \mathrm{C}$ by a chiller. All the coil dimensions were optimized by theoretical calculations, where we looked for the lowest inductance and resistance. In the end, we reduced time response of the magnetic field again to $1 \mathrm{~ms}$ by using the feedback control circuit. Its temperature stabilizes, after 5 minutes, at $60^{\circ} \mathrm{C}$ when we fed it with $40 \mathrm{~A}$.

The blue and red lasers optical table were improved as well to avoid high power losses, mainly due to dichroic optics. Now, the superposition of blue and red trap beams is done closer to the science chamber by independent optics, which allow us to save 60 $\mathrm{mW}$ in the blue laser system and have more light to trap atoms in the narrow transition. In the blue laser system, the main work were concerned to the spectroscopy cell and the use of its signal in the commercial Toptica's Digilock to stabilize the blue laser light.

The red laser system by itself is a challenge. We improved the alignment and mode-match of the master red to the ultra-stable cavity which permit us to lock it to the cavity, via PDH technique. This kind of stability provides us stable resonant red light, emitted by the slave laser stabilized by injection lock, for the same amount of time.

Another point in this Ph.D. is the development of new homemade apparatus in order to reduce the high cost of an cold atoms experiment and, as well, improve the current generation of this experiment. In this scenario, the cited laser diode housing with an improved temperature stabilization and confocal scanning etalon are only two examples of a countless projects designed.

From this hard work, we could reach two strong results. The first one is the demonstration of dramatic linewidth narrowing by injection lock of an low cost $461 \mathrm{~nm}$ diode laser and its application to our strontium experiment.

The second one is the new scattering effect, which is the observation and characterization of mCBS (mirror assisted coherent backscattering). This effect can be observed at low optical densities, which allows for example to work in very dilute clouds or far from resonance. Alson, due to the fact that the radiation of an atom interferes constructively with that of its own image, the phenomenon is much more robust to dephasing induced by strong saturation. More remarkable, the contribution of inelastic photons to the interference process can be demonstrated. 



\section{References}

1 CHANELIÈRE, T. et al. Saturation-induced coherence loss in coherent backscattering of light. Physical Review E, v. 70, p. 036602, 2004.

2 LABEYRIE, G. Coherent transport of light in cold atoms. Modern Physics Letters B, v. 22, n. 02, p. 73-99, 2008.

3 DAVIS, K. B. et al. Bose-Einstein condensation in a gas of sodium atoms. Physical Review Letters, v. 75, p. 3969-3973, 1995.

4 MOduGNO, G. et al. Bose-Einstein condensation of potassium atoms by sympathetic cooling. Science, v. 294, n. 5545, p. 1320-1322, 2001.

5 ANDERSON, M. H. et al. Observation of Bose-Einstein condensation in a dilute atomic vapor. Science, v. 269, n. 5221, p. 198-201, 1995.

6 SENGSTOCK, K. et al. Optical Ramsey spectroscopy on laser-trapped and thermal mg atoms. Applied Physics B, v. 59, n. 2, p. 99-115, 1994.

7 GRÜNERT, J.; HEMMERICH, A. Sub-Doppler magneto-optical trap for calcium. Physical Review A, v. 65, p. 041401, 2002.

8 KATORI, H. et al. Magneto-optical trapping and cooling of strontium atoms down to the photon recoil temperature. Physical Review Letters, v. 82, p. 1116-1119, 1999.

9 DICKE, R. H. Coherence in spontaneous radiation processes. Physical Review, v. 93, p. $99-110,1954$.

10 SCULLY, M. O.; SVIDZINSKY, A. A. The effects of the $n$ atom collective Lamb shift on single photon superradiance. Physics Letters A, v. 373, n. 14, p. 1283 - 1286, 2009.

11 RÖHLSBERGER, R. et al. Collective Lamb shift in single-photon superradiance. Science, v. 328, n. 5983, p. 1248-1251, 2010.

12 FRIEDBERG, R.; HARTMANN, S.; MANASSAH, J. Frequency shifts in emission and absorption by resonant systems ot two-level atoms. Physics Reports, v. 7, n. 3, p. $101-179,1973$. 
13 WATSON, K. M. Multiple scattering of electromagnetic waves in an underdense plasma. Journal of Mathematical Physics, v. 10, n. 4, p. 688-702, 1969.

14 ALBADA, M. P. V.; LAGENDIJK, A. Observation of weak localization of light in a random medium. Physical Review Letters, v. 55, p. 2692-2695, 1985.

15 WOLF, P.-E.; MARET, G. Weak localization and coherent backscattering of photons in disordered media. Physical Review Letters, v. 55, p. 2696-2699, 1985.

16 PHILLIPS, W. D. Nobel lecture: laser cooling and trapping of neutral atoms. Reviews of Modern Physics, v. 70, p. 721-741, 1998.

17 COHEN-TANNOUDJI, C. N. Nobel lecture: manipulating atoms with photons. Reviews of Modern Physics, v. 70, p. 707-719, 1998.

18 LETT, P. D. et al. Optical molasses. Journal of the Optical Society of America B, v. 6, n. 11, p. 2084-2107, 1989.

19 CASTIN, Y.; WALLIS, H.; DALIBARD, J. Limit of Doppler cooling. Journal of the Optical Society of America B, v. 6, n. 11, p. 2046-2057, 1989.

20 WINELAnD, D. J.; ITANO, W. M. Laser cooling of atoms. Physical Review A, v. 20, p. 1521-1540, 1979.

21 METCALF, H.; STRATEN, P. van der. Laser cooling and trapping. New York: Springer Verlag, 2012. 324 p. (Graduate Texts in Contemporary Physics). ISBN 9781461214700 .

22 ADAMS, C.; RIIS, E. Laser cooling and trapping of neutral atoms. Progress in Quantum Electronics, v. 21, n. 1, p. 1 - 79, 1997.

23 PRITCHARD, D. E. et al. Light traps using spontaneous forces. Physical Review Letters, v. 57, p. 310-313, 1986.

24 PHILLIPS, W. D.; METCALF, H. Laser deceleration of an atomic beam. Physical Review Letters, v. 48, p. 596-599, 1982.

25 BAGNATO, V.; ASPECT, A.; ZILIO, S. Study of laser deceleration of an atomic beam by monitoring the fluorescence along the deceleration path. Optics Communications, v. 72, n. 1-2, p. $76-81,1989$. 
26 KOGELNIK, H.; LI, T. Laser beams and resonators. Applied Optics, v. 5, n. 10, p. 1550-1567, 1966.

27 DREVER, R. W. P. et al. Laser phase and frequency stabilization using an optical resonator. Applied Physics B, v. 31, n. 2, p. 97-105, 1983.

28 CHANELIÈRE, T. et al. Three dimensional cooling and trapping with a narrow line. The European Physical Journal D, v. 46, n. 3, p. 507-515, 2008.

29 STELlmeR, S. et al. Bose-Einstein condensation of strontium. Physical Review Letters, v. 103, p. 200401, 2009.

30 STELLMER, S. et al. Bose-Einstein condensation of ${ }^{86} \mathrm{Sr}$. Physical Review A, v. 82, p. $041602,2010$.

31 STELLMER, S. et al. Laser cooling to quantum degeneracy. Physical Review Letters, v. 110, p. 263003, 2013.

32 YE, J.; CUNDIFF, S. Femtosecond optical frequency comb: principle, operation and applications. New York: Springer Verlag, 2006. 362 p. ISBN 9780387237916.

33 KUROSU, T.; SHIMIZU, F. Laser cooling and trapping of calcium and strontium. Japanese Journal of Applied Physics, v. 29, n. 11A, p. L2127, 1990.

34 ESCOBAR, Y. N. M. de et al. Bose-Einstein condensation of ${ }^{84}$ Sr. Physical Review Letters, v. 103, p. 200402, 2009.

35 KATORI, H. et al. Ultrastable optical clock with neutral atoms in an engineered light shift trap. Physical Review Letters, v. 91, p. 173005, 2003.

36 NICHOLSON, T. et al. Systematic evaluation of an atomic clock at $2 \times 10^{-18}$ total uncertainty. Nature, v. 6, n. 6896, p. 1320-1322, 2015.

37 SIEGMAN, A. Lasers. New York: University Science Books, 1986. 1283 p. ISBN 9780935702118.

38 PANTELL, R. The laser oscillator with an external signal. Proceedings of the Institute of Electrical and Electronics Engineers, v. 53, n. 5, p. 474-477, 1965. 
39 TANG, C. L.; STATZ, H. Phase-locking of laser oscillators by injected signal. Journal of Applied Physics, v. 38, n. 1, p. 323-324, 1967.

40 STOVER, H. L.; STEIER, W. H. Locking of laser oscillators by light injection. Applied Physics Letters, v. 8, n. 4, p. 91-93, 1966.

41 BUCZEK, C.; FREIBERG, R.; SKOLNICK, M. Laser injection locking. Proceedings of the Institute of Electrical and Electronics Engineers, v. 61, n. 10, p. 1411-1431, 1973.

42 WINKELMANN, L. et al. Injection-locked single-frequency laser with an output power of 220 w. Applied Physics B, v. 102, n. 3, p. 529-538, 2011.

43 SHIMADA, Y. et al. A simplified 461-nm laser system using blue laser diodes and a hollow cathode lamp for laser cooling of sr. Review of Scientific Instruments, v. 84, n. 6, 2013.

44 COURTILLOT, I. et al. Efficient cooling and trapping of strontium atoms. Optics Letters, v. 28, n. 6, p. 468-470, 2003.

45 KUGA, Y.; ISHIMARU, A. Retroreflectance from a dense distribution of spherical particles. Journal of the Optical Society of America A, v. 1, n. 8, p. 831-835, 1984.

46 WIERSMA, D. S. et al. Experimental evidence for recurrent multiple scattering events of light in disordered media. Physical Review Letters, v. 74, p. 4193-4196, 1995.

47 YOO, K. M.; TANG, G. C.; ALFANO, R. R. Coherent backscattering of light from biological tissues. Applied Optics, v. 29, n. 22, p. 3237-3239, 1990.

48 MISHCHENKO, M. On the nature of the polarization opposition effect exhibited by saturn,s rings. Astrophysical Journal, v. 411, n. 1, p. 351-361, 1993.

49 TOURIN, A. et al. Time-dependent coherent backscattering of acoustic waves. Physical Review Letters, v. 79, p. 3637-3639, 1997.

50 LABEYRIE, G. et al. Coherent backscattering of light by cold atoms. Physical Review Letters, v. 83, p. 5266-5269, 1999.

51 KUPRIYANOV, D. V. et al. Coherent backscattering of light in atomic systems: application to weak localization in an ensemble of cold alkali-metal atoms. Physical Review A, v. 67, p. 013814, 2003. 
52 KUPRIYANOV, D. V. et al. Coherent backscattering of light from ultracold and optically dense atomic ensembles. Laser Physics Letters, v. 3, n. 5, p. 223, 2006.

53 BIDEL, Y. et al. Coherent light transport in a cold strontium cloud. Physical Review Letters, v. 88, p. 203902, 2002.

54 MÜLLER, C. A. et al. Weak localization of light by cold atoms: the impact of quantum internal structure. Physical Review A, v. 64, p. 053804, 2001.

55 MÜLLER, C. A. et al. Mesoscopic scattering of spin $s$ particles. Journal of Physics $A$, v. 38, n. 36, p. $7807,2005$.

56 JONCKHEERE, T. et al. Multiple scattering of light by atoms in the weak localization regime. Physical Review Letters, v. 85, p. 4269-4272, 2000.

57 KAISER, R.; HAVEY, M. D. Mesoscopic electromagnetic wave dynamics in ultracold atomic gases. Optics and Photonics News, v. 16, n. 7, p. 38-43, 2005.

58 TOTSUKA, K.; TOMITA, M. Coherent backscattering in a disordered optical medium in the presence of saturation absorption. Physical Review B, v. 59, p. 11139-11142, 1999.

59 WELLENS, T. et al. Coherent backscattering of light by two atoms in the saturated regime. Physical Review A, v. 70, p. 023817, 2004.

60 WELLENS, T. et al. Coherent backscattering of light by nonlinear scatterers. Physical Review E, v. 71, p. 055603, 2005.

61 ShatokHin, V.; MÜlleR, C. A.; BUCHLEITNER, A. Coherent inelastic backscattering of intense laser light by cold atoms. Physical Review Letters, v. 94, p. 043603, 2005.

62 GRÉMAUD, B. et al. Coherent backscattering in nonlinear atomic media: quantum langevin approach. Physical Review A, v. 74, p. 033808, 2006.

63 WELLENS, T. et al. Coherent backscattering of light with nonlinear atomic scatterers. Physical Review A, v. 73, p. 013802, 2006.

64 OTT, J. R. et al. Cooperative fluorescence from a strongly driven dilute cloud of atoms. Physical Review A, v. 87, p. 061801, 2013. 
65 KETTERER, A.; BUCHLEITNER, A.; SHATOKHIN, V. N. Coherent inelastic backscattering of laser light from three isotropic atoms. Physical Review A, v. 90, p. 053839, 2014.

66 GREFFET, J.-J. Backscattering of s-polarized light from a cloud of small particles above a dielectric substrate. Waves in Random Media, v. 1, n. 3, p. S65-S73, 1991.

67 BONIFACIO, R.; SALVO, L. D. Collective atomic recoil laser (carl) optical gain without inversion by collective atomic recoil and self-bunching of two-level atoms. Nuclear Instruments and Methods in Physics Research, v. 341, n. 1-3, p. 360-362, 1994.

68 LABEYRIE, G. et al. Optomechanical self-structuring in a cold atomic gas. Nature Photonics, v. 8, n. 4, p. 321-325, 2014.

69 INOUYE, S. et al. Superradiant rayleigh scattering from a Bose-Einstein condensate. Science, v. 285, n. 5427, p. 571-574, 1999.

70 SCULLY, M. O. et al. Directed spontaneous emission from an extended ensemble of $n$ atoms: Timing is everything. Phys. Rev. Lett., v. 96, p. 010501, 2006.

71 CIARAMELlA, E.; TAMBURRINI, M.; SANTAMATO, E. Talbot assisted hexagonal beam patterning in a thin liquid crystal film with a single feedback mirror at negative distance. Applied Physics Letters, v. 63, n. 12, p. 1604-1606, 1993.

72 STEINER, I.; TOSCHEK, P. E. Quenching quantum phase noise: correlated spontaneous emission versus phase locking. Physical Review Letters, v. 74, p. 4639-4642, 1995.

73 ESCHNER, J. et al. Light interference from single atoms and their mirror images. Nature, v. 413, n. 6855, p. 495-498, 2001.

74 SCULLY, M.; ZUBAIRY, M. Quantum optics. Cambridge: Cambridge University Press, 1997. 656 p. ISBN 9780521435956.

75 BIENAIMé, T. et al. Atom and photon measurement in cooperative scattering by cold atoms. Journal of Modern Optics, v. 58, n. 21, p. 1942-1950, 2011.

76 MÁXIMO, C. E. et al. Atomic lighthouse effect. Journal of the Optical Society of America A, v. 31, n. 11, p. 2511-2517, 2014.

77 COURTEILLE, P. W. et al. Modification of radiation pressure due to cooperative scattering of light. The European Physical Journal D, v. 58, n. 1, p. 69-73, 2010. 
78 HILL, I. R. et al. Zeeman slowers for strontium based on permanent magnets. Journal of Physics B, v. 47, n. 7, p. 075006, 2014.

79 LEBEDEV, V.; WELD, D. M. Self-assembled zeeman slower based on spherical permanent magnets. Journal of Physics B, v. 47, n. 15, p. 155003, 2014.

80 YU, D. Photonic band structure of the three-dimensional ${ }^{88} \mathbf{S r}$ atomic lattice. Physical Review A, v. 84, p. 043833, 2011. 

Appendix 



\section{APPENDIX A - Toptica Digilock module}

The DL Pro diode laser has four methods of frequency tuning: a coarse screw adjusting the angle of feedback grating, a piezo providing fine adjustment of the grating, temperature of semiconductor, and the injection current. The grating angle selects the frequency which is reflected back into the laser cavity. The properties of the diode control the semiconductor band gap. While tuning the temperature is very useful for reaching the appropriate laser frequency and achieving single mode operation, due to thermal inertia it allows a slower response time than either modulation of the piezo or injection current. Thus the temperature is stabilized using it is own PID controller.

This leaves two controls for stabilizing the laser frequency to the atomic resonance. The injection current can be controlled on fast time scales as, unlike the piezo, it is not limited by mechanical motion. The two are therefore typically used in conjunction to stabilize over two different frequency bands - the current ensuring stability on short timescales and the piezo preventing long-term drifts.

The Digilock module allows use of two PID controllers and one would typically be assigned to each control. However, in the current control configuration of the laser the short-term drift is controlled by the second harmonic generation feedback system, using the injection modulation input. Therefore, for initial locking a single PID controller is used to stabilize long-term drifts in frequency through control over the piezo.

Locking is performed using either one of the two PID controllers to lock to a setpoint directly, or the Autolock to control one or both PID controllers to achieve locking on a set-point. In our case we would want to lock on the minima of the Lamb dip. To create a stable lock the Digilock can be used to create a derivative error signal through using the Lock-in control (it also has Pound-Drever-Hall). This adds a user set modulation onto the laser frequency. The resulting derivative signal should then be fed into the Autolock system (and therefore the PID controllers) to provide the appropriate set-point for locking.

\section{A.1 Overview of software and functionality}

On switching on (and connecting to the appropriate Digilock module from the software server) the module allows computer control over the SCC110 piezo controller module via a connection in the back-plane of the Toptica electronics rack. The user can then control the offset, scan range and scan frequency from the computer. Note that the offset and range (labeled SCC110 out) are cumulative with the settings on the SCC110 front panel, while the scan frequency is overridden by the Digilock (but takes the range from the front panel). Fig. 48 shows the user interface. The lower portion of the GUI functions as an oscilloscope with two input channels and a time axis represented by the piezo position during scan. This should be used to observe the spectroscopy photodiode signal (labeled Main in) and the derivative signal (labelled LI out). The settings for the Autolock module, PID controllers and Lock-In should be modified and wired correctly on their appropriate tabs. The PID controllers can have their block-diagrams rewired in the 
virtual back-end of the software, allowing more optimisation if necessary. See Table 5 for the correct module hierarchy and reasonable settings.

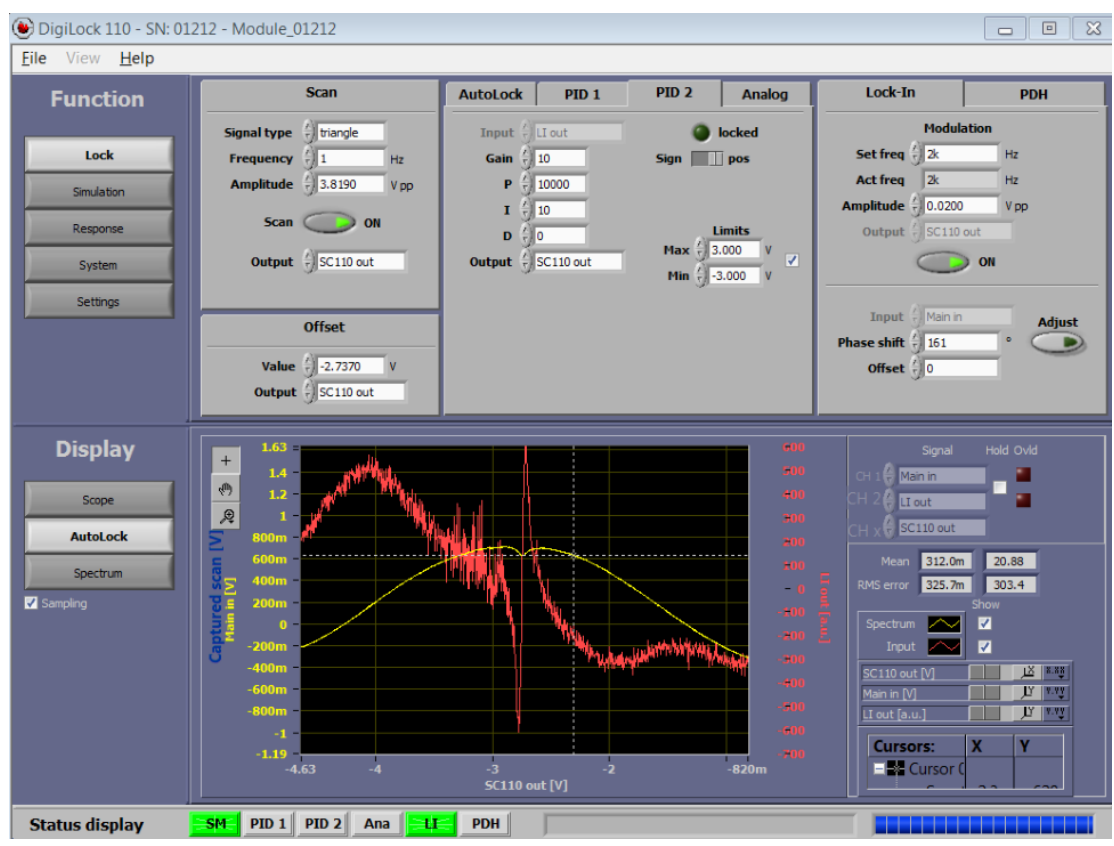

Figure 48 - Showing the user interface with reasonable settings, and displaying a usable derivative signal. The Scan tab allows control over the SCC110 module. The top middle section is used to set up the PID controllers and Autolock heirarchies. On the top right is the Lock-In control when the settings used for generating a derivative signal can be controlled. The lower portion is a dedicated oscilloscope. The Scope tab shows the direct values of the chosen inputs (Main In and LI out in the image). The Autolock tab shows the inputs over the Autolock range, and allows direct manipulation of the SCC110 (i.e. zooming and panning changes the scan range and scan offset respectively). The dotted line on the oscilloscope is the Autolock cursor - to lock you should position this at the locking point, then right click and select lock. Two useful points: 1) if the oscilloscope stops refreshing make sure Sampling is ticked, and: 2) in the lower left there are three rows which have the channel names in them. They are followed by three boxes - clicking on these boxes turns on and off auto-scaling. Source: By the author.

In order to achieve a lock using Lock In derivative signal, the following steps should be followed:

1. Using the SCC110 front panel scan offset to reach the center laser frequency found in Table 2 and increase the scan amplitude to obtain a clear picture of the spectroscopy absorption spectrum on oscilloscope. Ensure that the laser is at the correct frequency and that no mode hops appear on the spectrum (sudden, sharp and usually short drops/increases in intensity);

2. Switch on Digilock module and connect using software server. This will change the scan offset, adjust the Digilock scan offset value until back at the correct center frequency. Ensure that the Digilock oscilloscope channels are set to Main in and LI out. Reduce the Digilock scan frequency until you have one full absorption spectrum showing on the Digilock virtual oscilloscope. It may be necessary to change the frequency range on the SCC110 front panel; 
Table 5 - Summary of settings for the Digilock interface.

\begin{tabular}{|c|c|c|}
\hline Module & Property & Value \\
\hline \multirow[t]{3}{*}{ SCC110 } & Center frequency & $325.25185 \mathrm{THz}$ \\
\hline & Scan amplitude & spectrum: $3 \mathrm{~V}$, dip: $0.2 \mathrm{~V}$ \\
\hline & Scan frequency & $1 \mathrm{~Hz}$ \\
\hline \multirow[t]{5}{*}{ Lock in } & Input & Main in \\
\hline & Modulation frequency & $2 \mathrm{kHz}$ \\
\hline & Modulation amplitude & $0.02 \mathrm{~V}_{p p}$ \\
\hline & Phase & $160^{\circ}$ \\
\hline & Offset & 0 \\
\hline \multirow[t]{2}{*}{ Autolock } & Input & LI Out \\
\hline & PIDs active & PID 2 \\
\hline \multirow[t]{5}{*}{ PID 2} & Output & SCC110 out \\
\hline & G & 10 \\
\hline & $\mathrm{P}$ & 10000 \\
\hline & I & 10 \\
\hline & $\mathrm{D}$ & 0 \\
\hline
\end{tabular}

Source: By the author.

3. Remove scan amplitude on SCC110 and increase on Digilock until full absorption spectrum is recovered. An amplitude of $3 \mathrm{~V}$ should be sufficient;

4. Ensure that the Lock-In, Autolock and PID modules have their inputs and outputs defined as in Table 5. (If also using PID 1 it should be set to control the injection current, labelled Main out);

5. Set the Lock-In module settings to the values of Table 5. Optimize the modulation frequency and amplitude to have a straight and as noise free as possible derivative signal over the Lamb dip, Fig. 48 shows a typical signal on the oscilloscope. Set the phase by pressing the Adjust tab to auto-optimize;

6. Set PID 2 to the settings. These gave lock times of over 10 minutes, and I have not seen the laser drop out of lock without a mode hop. If wanting to optimize it may be better to start from PID 2 settings of Gain $10, P=100, I=10$ and $D=0$;

7. Switch the oscilloscope to the Autolock channel. Reduce Digilock scan amplitude by zooming in/panning along the x-axis until you can clearly see the derivative signal over Lamb dip (it may be necessary to re-adjust the phase). Click and drag the cursor to the center of the Lamb dip. Right click and select lock to extremum. It does not matter if the cursor follows the absorption spectrum, the Autolock will lock onto the derivative signal at the same point on the $\mathrm{x}$-axis;

8. Check frequency is stable on wavelength meter and optimize PID settings of PID 2 if necessary. 


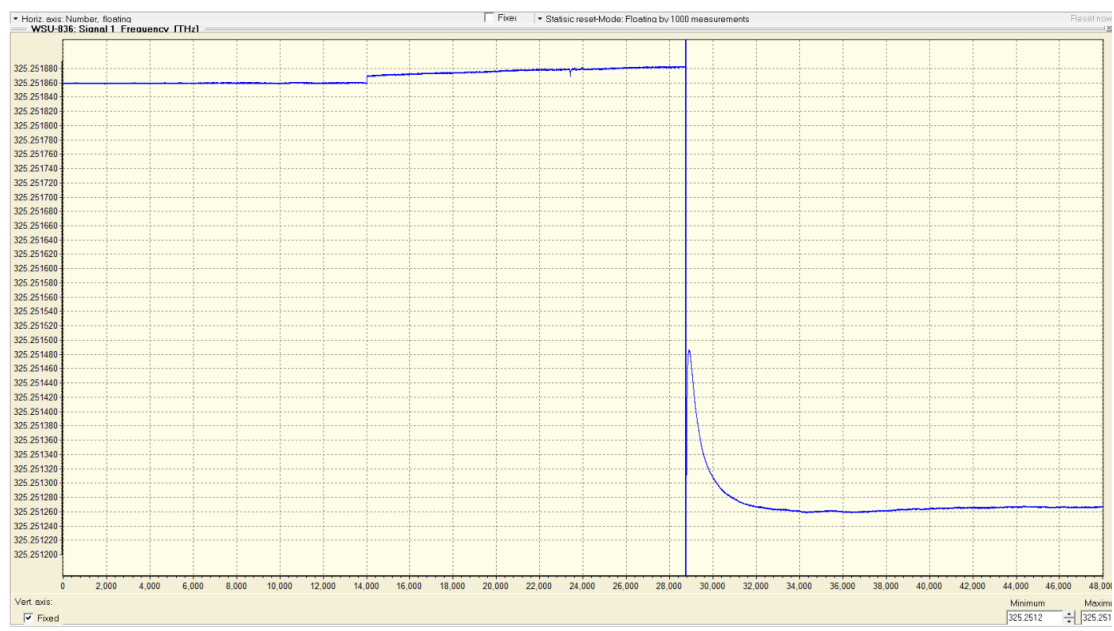

Figure 49 - Showing frequency versus time for the locked laser, free running laser and free running with the Digilock module switched off. One minute corresponds to 6000 measurements. Measured using High-finesse WSU wavelength meter. Source: By the author.

By the author. 